\title{
Combustion synthesis of fullerenes and fullerenic nanostructures
}

by

\author{
Anish Goel \\ B.S.E. in Chemical Engineering \\ University of Michigan, 1997 \\ M.S. in Chemical Engineering Practice \\ Massachusetts Institute of Technology, 1999
}

Submitted to the Department of Chemical Engineering in partial fulfillment of the requirements for the degree of

Doctor of Philosophy

at the

Massachusetts Institute of Technology

June 2002

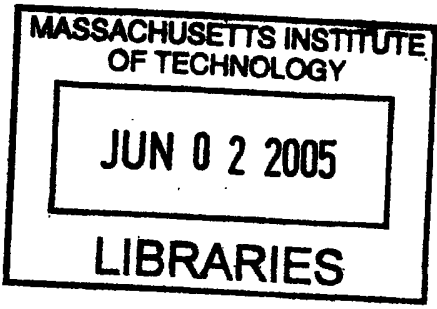

(C) 2002 Massachusetts Institute of Technology

All rights reserved

Signature of Author:

Department of Chemical Engineering

March 2002

Certified by:

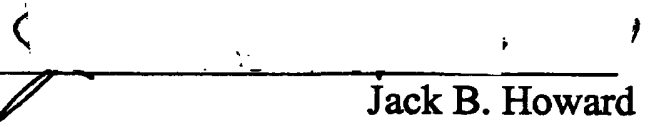

Hoyt C. Hottel Professor of Chemical Engineering

Thesis Supervisor

Accepted by:

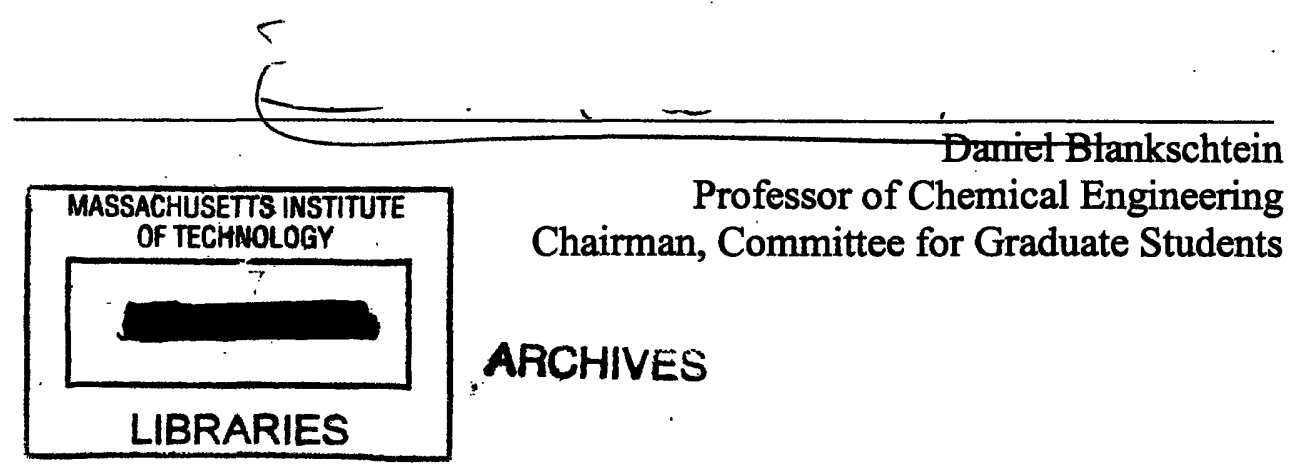




\title{
Combustion synthesis of fullerenes and fullerenic nanostructures
}

\author{
by Anish Goel
}

Submitted to the Department of Chemical Engineering on 21 March 2002 in partial fulfillment of the requirements for the degree of Doctor of Philosophy in Chemical Engineering

\begin{abstract}
Fullerenes are molecules comprised entirely of $\mathrm{sp}^{2}$-bonded carbon atoms arranged in pentagonal and hexagonal rings to form a hollow, closed-cage structure. Buckyballs, a subset which contains $\mathrm{C}_{60}$ and $\mathrm{C}_{70}$, are single-shell molecules while fullerenic nanostructures can contain many shells and over 300 carbon atoms. Both fullerenes and nanostructures have an array of applications in a wide variety of fields, including medical and consumer products. Fullerenes were discovered in 1985 and were first isolated from the products of a laminar low-pressure premixed benzene/oxygen/argon flame operating at fuel-rich conditions in 1991. Flame studies indicated that fullerene yields depend on operating parameters such as temperature, pressure, residence time, and equivalence ratio. High-resolution transmission electron microscopy (HRTEM) showed that the soot contains nanostructures, including onions and nanotubes.

Although flame conditions for forming fullerenes have been identified, the process has not been optimized and many flame environments of potential interest are unstudied. Mechanistic characteristics of fullerene formation remain poorly understood and cost estimation of large-scale production has not been performed. Accordingly, this work focused on: 1) studying fullerene formation in diffusion and premixed flames under new conditions to identify optimal parameters; 2) investigating the reaction of fullerenes with soot; 3) positively identifying $\mathrm{C}_{60}$ molecules in HRTEM by tethering them to carbon black; and 4) providing a cost estimation for industrial fullerenic soot production.

Samples of condensable material from laminar low-pressure benzene/argon/oxygen diffusion flames were collected and analyzed by high-performance liquid chromatography (HPLC) and HRTEM. The highest concentration of fullerenes in a flame was always detected just above the height where the fuel is consumed. The percentage of fullerenes in condensable material increases with decreasing pressure and the fullerene content of flames with similar cold gas velocities shows a strong dependence on length. A shorter flame, resulting from higher dilution or lower pressure, favors the formation of fullerenes rather than soot, exhibited by the lower amount of soot and precursors in such flames. This indicates a stronger correlation of fullerene consumption to soot levels than of fullerene formation to precursor concentration. The maximum flame temperature seems to be of minor importance in formation. The overall highest amount of fullerenes was found for a surprisingly high dilution of fuel with argon. The HRTEM analysis showed an increase of the curvature of the carbon layers, and hence increased fullerenic character, with increasing distance from the burner up to the point of maximum fullerene concentration, after which it decreases, consistent with the HPLC analysis. The soot shows highly ordered regions that appear to have been cells of fullerenic nanostructure formation. The samples also included fullerenic nanostructures such as tubes and spheroids including highly-ordered multilayered or onion-like structures. Studies of turbulent-like benzene/oxygen/argon diffusion flames showed that these flames produce
\end{abstract}


fullerenes over a wider range of heights than laminar flames but with lower yields. No discernible trend could be detected in the data and the fullerene results were not easily reproducible indicating that such flames are not suitable for fullerene formation.

Soot samples were also collected from a well-characterized laminar premixed benzene/oxygen/argon flat flame under new conditions and analyzed by HPLC and HRTEM. Flame studies using secondary injections of benzene or acetylene show that two-stage flames are unsuitable for fullerene production. It seems that secondary fuel has an adverse effect on the formation of fullerenes and creates conditions that are similar to the early stages of a single-stage flame prior to soot formation. This means that fuel must go through the combustion process to form fullerenes and that they cannot be formed simply by organic pyrolysis. Additionally, fullerene data collected in this study show significantly higher yields than in a previous study and the absence of a concentration drop-off. The coexistence of fullerenes and soot does not support but also does not rule out that fullerenes are consumed by soot, as was suggested by diffusion flame data.

Given the discrepancy in the data, fullerene consumption was studied in experiments involving pure fullerenes being sublimated into a passing argon gas stream. This gas stream then passed through a carbon black bed. As the fullerenes passed through the bed, a certain percentage reacted with the surface of the particles and the non-reacted material was collected downstream. Experiments at different temperatures indicate that fullerenes are indeed consumed by soot particles but that the consumption is quite slow. The rate coefficient obtained resembles those seen for surface diffusion controlled reactions or for heterogenous reactions. Extrapolation of the reaction coefficient to flame conditions would indicated that this type of fullerene consumption is not nearly enough to explain the consumption observed in fullerene-forming flames, meaning that fullerenes are consumed by other mechanisms.

HRTEM analysis of carbon black with and without tethered fullerenes shows that fullerenes can in fact be observed in TEM micrographs. In this experiment, functionalized $\mathrm{C}_{60}$ molecules were attached to the surface of carbon black particles with a chemical tether. The resulting compound was analyzed by HRTEM and compared with similar analysis of untreated carbon black. The post-treatment carbon black not only has an order of magnitude greater concentration of apparent fullerene structures but size distribution data shows a significant peak at the $\mathrm{C}_{60}$ diameter for the treated sample whereas no peak is observed for the untreated sample. This indicates that the fullerenes have indeed been attached to the particle surface and that they can definitively be seen in images produced from HRTEM.

Lastly, a model was built to estimate the cost of the large scale production of fullerenic soot. This model was based on current carbon black technology and takes into account operating parameters specific for fullerene production. Sensitivity analyses performed on the model indicate that soot yield and fuel price are the most important factors in determining production cost while electricity costs are minimally important. It was seen that operating pressure and equipment lifetime are negligible in the final cost.

Overall, combustion holds immense promise to be a much cheaper and more efficient alternative to the current method of commercial fullerene production.

Thesis Supervisor: Jack B. Howard

Title:

Hoyt C. Hottel Professor of Chemical Engineering 
to

ma, pa, \& rink

who have catapulted me above the clouds and allowed me to touch the sky 


\section{Acknowledgements}

Although many of my partners in crime would prefer to remain blissfully anonymous, I will nevertheless try to identify as many as my weary mind will allow. I apologize in advance for any omissions as they are purely unintentional. The folks mentioned here are primarily responsible for my successes but all the mistakes and shortcomings are completely my own.

I of course owe a great deal to my advisor, Prof. Jack Howard. Whether in combustion or the world at-large, his constructive support, dignified approach, and walking authority have set an example that I can only hope to live up to. His imminent departure from MIT reminds me how fortunate I've been to have had the opportunity to work with such a legendary and respected individual. Like a seasoned jedi with his novice apprentice, he has guided me in the right direction yet allowed me to search out my own path. I wish I knew a bigger word than 'thanks'.

For undertaking the unenviable task of serving on my thesis committee, I am indebted to Prof. John Vander Sande, Dr. Art Lafleur, and Prof. Bill Green. All have made valuable contributions and suggestions when they were sorely needed. I especially recognize Prof. Vander Sande, joined by his masters of TEM Lenore Rainey, Paulo Ferreira, and Paula Jardim, for their boundless insight and enthusiasm on a plethora of TEM images and collaborations. The expert chemical analyses provided by Koli Taghizadeh, Elaine Plummer, and John LaRusso were similarly invaluable to me and Peter Morley brought all my half-baked designs to vibrant life. Profs. Alan Hatton and Jefferson Tester also deserve special thanks for keeping me happy when I was mired in the bureaucracies of the department.

For my buddies in the basement, my illustrious colleagues of fire, the dungeon dwellers of Building 66, what can I say? Their discussions, insight, intrigue, and general merriment have sustained me during this arduous trek. Without them, this thesis would be postponed indefinitely. Members current and former include Murray Height, Patty Sullivan, Henning Richter, Mike Timko, Gerardo Ferreiro, Jin Qian, Heather Stern, Jason Ploeger, Liz Gron, Peter Hebgen, Isidro Griful, Josh Taylor, David Kronholm, Joe Schanzenbecher, Quico Ayala, Wolfgang Voelcker, Thilo Lehre, and Tony Modestino. I will sadly miss our many moons of shared jubilation and commiseration.

I am also beholden to a group I call the 'circle of power' who have helped me in ways that go beyond research and academics. Ilea Mathis, Anna Di Maria, Craig Abernethy, Carol Phillips, Arline Benford, Susan Lanza, Barbara Driscoll, Patsy Sampson, Emmy Snyder, Suzanne Easterly, Annie Fowler, and Elaine Aufiero do not possess the title of professor, but in most ways, they have more authority - a student couldn't ask for a more devoted bunch. I owe them my livelihood at bonny MIT.

I could never adequately express my appreciation for my family. Always a bedrock of support and encouragement and the source of countless examples to follow, they are titans of integrity, generosity, and success and I am but a mouse, struggling to keep up. They have known me better than I have known myself - never believing (and never letting me forget) my now infamous senior-year comment, "They couldn't pay me enough to go to grad school." So to Mom, Dad, Anuj, and Stacy - you have helped me more than you can ever know.

And finally, to all my sanity-keepers over the years. Some are honorary dungeon dwellers and some have never set foot on MIT campus, but they have all helped me in special ways that can never be tangibilized. In no particular order, they are: Amy Kanellis, Randall Urbance, Brian Makins, Jannise Obst, David Matheu, Christa Beranek, David Collins, the indomitable Paul Fernandez, John O'Quinn, Wenny Jean, Mutsumi Yoshida, Jean Dubay, Sara Soderstrom, Jean Bender, Ian Das, Jamie Bechtel, Jim Bielenberg, Paul Yelvington, April Ross, Ley Richardson, Kim Bryan, Ian Zacharia, Kevin Dorfman, Audrey Johnson, Chris Martin, Eric Nelson, Chen Wang, Zhitao Cao, Thomas Epps, Gia Schneider, Javier Femenia, Erika Martin, Lacey Southerland, Shawn Cole, Ana Sala, Blanca San Miguel, Beatriz Escudero, Mariah Devereux, Silvia Roa, Brian Forster, Parag Desai, Vicki Green, Kym Ahrens, Nitin Mathur, Neil Aggarwal, and all my family in the homeland and abroad.

A last note of gratitude for the financial support from the National Aeronautics and Space Administration (Grant No. NAG3-1979) and their Graduate Student Researchers Program and from the Division of Chemical Sciences (Grant No. DE-FG02-84ER 13282), Office of Basic Energy Sciences, Office of Energy Research, United States Department of Energy.

Thank you and good night... 


\section{Table of Contents}

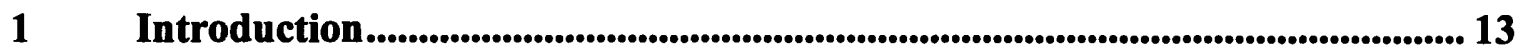

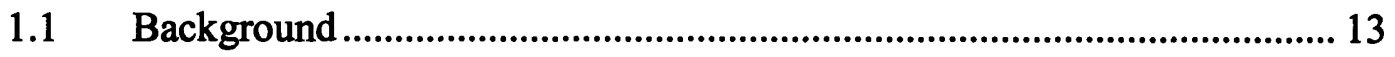

1.1.1 Formation of fullerenes and nanostructures.................................... 15

1.1.2 Fullerene properties and reactivity............................................... 17

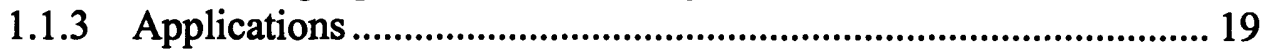

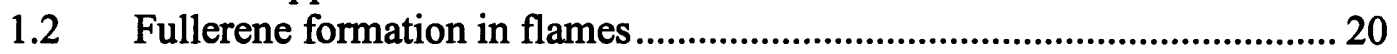

1.2.1 Methods of production................................................................... 20

1.2.2 Fullerene formation mechanisms............................................. 21

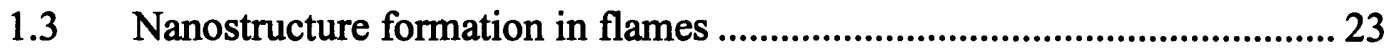

1.4 Commercial production of fullerenes ........................................................ 24

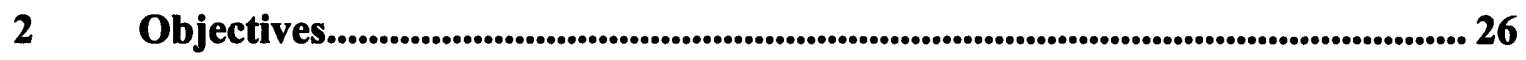

3 Diffusion flame studies ................................................................................. 27

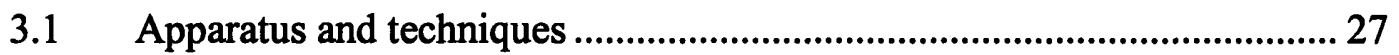

3.1.1 Flame chamber............................................................................ 27

3.1.1.1 Flame burners......................................................................28

3.1.1.2 Chamber sections .................................................................32

3.1.1.3 Top plate ………....................................................................34

3.1.2 Fuel delivery ............................................................................... 34

3.1.3 Quartz sampling probe ..................................................................... 35

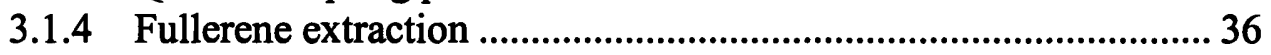

3.1.5 High-performance liquid chromatography ..................................... 37

3.1.6 High-resolution transmission electron microscopy ....................... 37

3.1.6.1 Qualitative analysis..........................................................37

3.1.6.2 Quantitative analysis.............................................................38

3.2 Low-flow laminar diffusion flames ......................................................... 39

3.2.1 Experimental conditions ................................................................ 39

3.2.2 Fullerene and soot results....................................................... 40

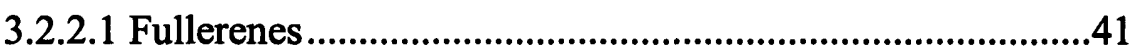

3.2.2.2 Soot structure evolution ...................................................44

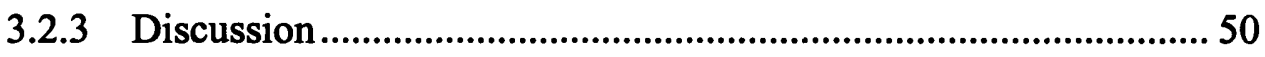

3.2.3.1 Fullerenes............................................................................50

3.2.3.2 Soot structure evolution ..................................................51

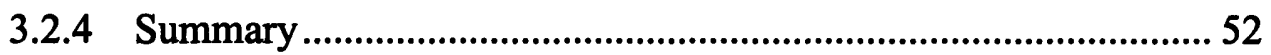

3.3 High-flow turbulent-like diffusion flames ...............................................5 53

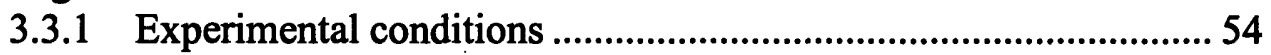

3.3.2 Fullerenes results ...................................................................... 55

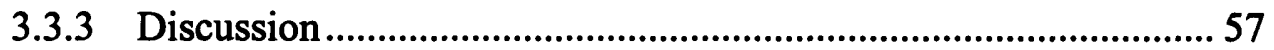

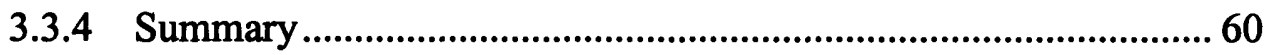

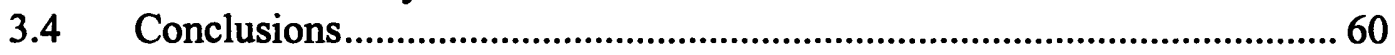

$4 \quad$ Premixed flame studies............................................................................................6 62

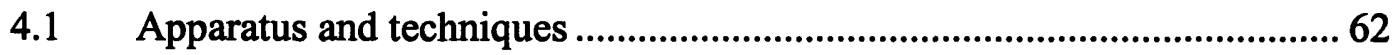

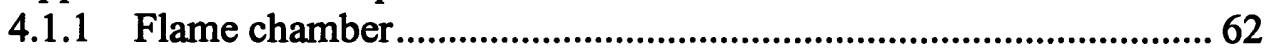


4.1.1.1 Flame burner ...................................................................63

4.1.1.2 Chamber bottom and extension .......................................65

4.1.1.3 Top plate ......................................................................66

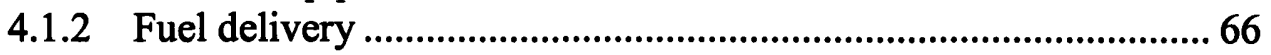

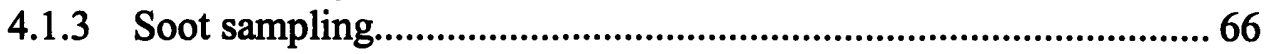

4.1.4 Extraction and analysis ....................................................... 67

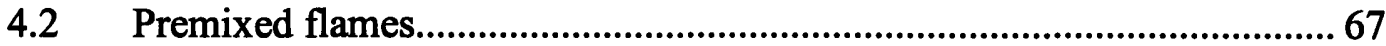

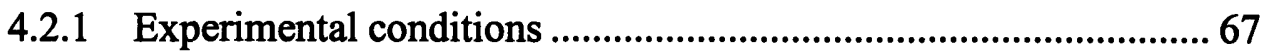

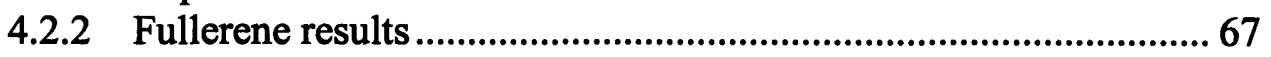

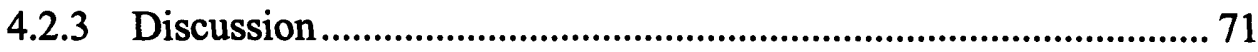

4.2.4 Summary ....................................................................... 73

4.3 Premixed flames with secondary injections......................................... 73

4.3.1 Experimental conditions ......................................................... 74

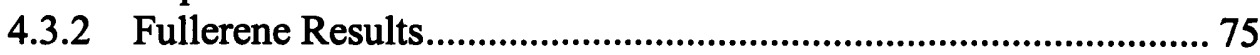

4.3.3 Discussion....................................................................... 77

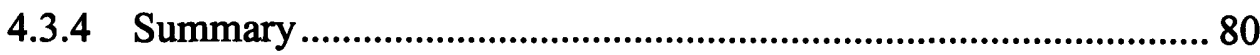

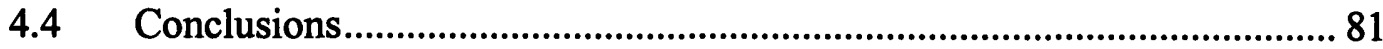

5 Reaction rate coefficient of fullerene $\left(\mathrm{C}_{60}\right)$ consumption by soot .................. 83

5.1 Experimental system and techniques .................................................... 84

5.1.1 Reactor and furnace setup.................................................... 84

5.1.2 Vapor collection.................................................................. 85

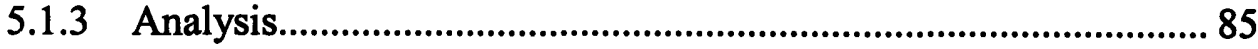

5.1.4 Experimental conditions ...................................................... 86

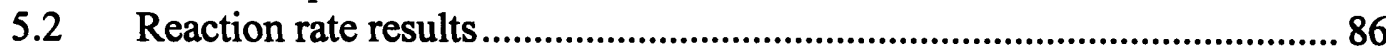

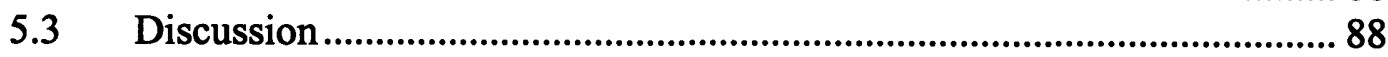

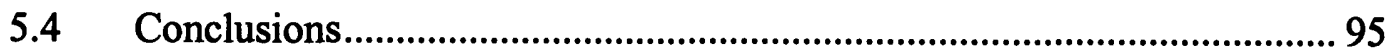

6 Observations of fullerenes tethered to carbon black particles ....................... 96

6.1 Experimental .................................................................................... 96

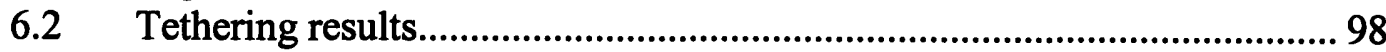

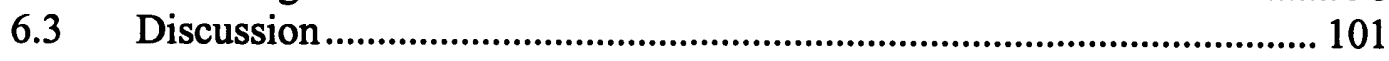

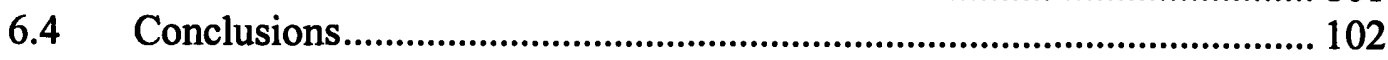

7 Economic cost modeling of commercial fullerenic soot plant....................... 103

7.1 Model basis ..................................................................................... 104

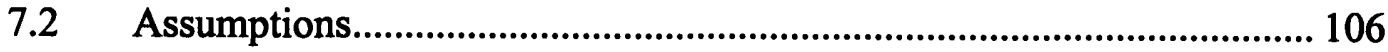

7.2.1 Incremental changes and additions ........................................ 106

7.2.2 Operating parameters ............................................................... 107

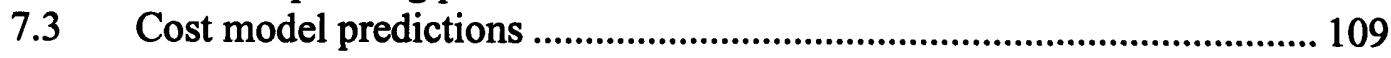

7.3.1 Base case cost estimate .......................................................... 110

7.3.2 Sensitivity to parameter adjustments ....................................... 111

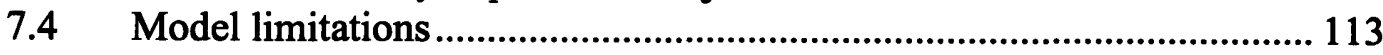

7.5 Implications for fullerene price and fullerenic soot industry................. 115 
9 Recommendations ............................................................................................................ 120

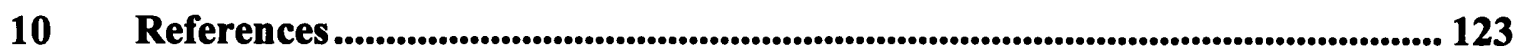

11 Appendices..................................................................................................................... 133

11.1 Appendix 1 - Tabulation of high-flow (turbulent) flame data ............... 133

11.2 Appendix 2 - Premixed flame data and sample calculation.................... 134

11.3 Appendix 3 - Calculations for fullerenic soot yield ................................. 136

11.3.1 Calculations from observed experimental data............................ 136

11.3.2 Thermodynamic equilibrium calculations by STANJAN............ 137

11.4 Appendix 4-Economic cost model for fullerenic soot plant ................. 139

11.4.1 Spreadsheet for economic cost model - base case cost ............... 139

11.4.2 Explanation of cost model ........................................................ 141 


\section{List of Figures}

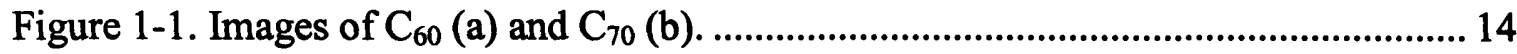

Figure 3-1. Diffusion flame chamber with burner and flame sampling probe. .................. 28

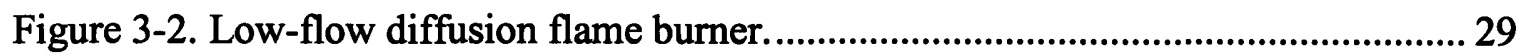

Figure 3-3. Burner caps with varying outlet diameters....................................................... 30

Figure 3-4. High-flow diffusion flame burner: side-view (a); top-view (b); and cut-away side-view with nozzle attached (c).

Figure 3-5. High-flow flame burner cover: side-view (a); and top-view (b).................... 32

Figure 3-6. Bottom section of diffusion flame chamber with burner and bottom plate. .. 33

Figure 3-7. One of two identical top sections of diffusion flame chamber. ........................ 33

Figure 3-8. Top plate of diffusion flame chamber........................................................... 34

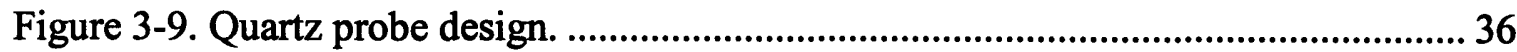

Figure 3-10. Method for quantitative analysis of TEM images........................................... 38

Figure 3-11. Quantity of fullerenes $\mathrm{C}_{60}$ and $\mathrm{C}_{70}$ in the condensable material (a) and concentration of fullerenes $\mathrm{C}_{60}$ and $\mathrm{C}_{70}$ in the cold probed gas (b) from different heights above the burner in different laminar diffusion flames at $\mathrm{p}=20$ Torr.

Figure 3-12. Quantity of fullerenes $\mathrm{C}_{60}$ and $\mathrm{C}_{70}$ in the condensable material (a) and concentration of fullerenes $\mathrm{C}_{60}$ and $\mathrm{C}_{70}$ in the cold probed gas (b) from different heights above the burner in different laminar diffusion flames at $\mathrm{p}=40$ Torr.

Figure 3-13. Maximum amount of fullerenes $C_{60}$ and $C_{70}(a)$ and mass ratio of $C_{60}$ to $C_{70}$ (b) in the condensable material at the height above the burner corresponding to maximum fullerene concentration in laminar diffusion flames, at different pressures ( $p$ ) and different argon dilutions ( $\left.\operatorname{dil}_{\mathrm{Ar}}\right)$.

Figure 3-14. Representative HRTEM image of material collected at $h=75 \mathrm{~mm}$ in flame $\# 11$ ( $\mathrm{p}=40$ Torr, $\mathrm{v}_{\mathrm{u}}($ fuel $\left./ \mathrm{Ar})=820 \mathrm{~cm} / \mathrm{s}, \mathrm{v}_{\mathrm{u}}(\mathrm{oxy})=3.65 \mathrm{~cm} / \mathrm{s}, \mathrm{dil}_{\mathrm{Ar}}=85.3 \%\right) . . .44$

Figure 3-15. Representative counted material from soot collected at $h=72 \mathrm{~mm}(a), 74 \mathrm{~mm}$ (b), $75 \mathrm{~mm}$ (c), and $77 \mathrm{~mm}$ (d) in flame \#11 ( $\mathrm{p}=40 \mathrm{Torr}, \mathrm{v}_{\mathrm{u}}(\mathrm{fuel} / \mathrm{Ar})=820$ $\left.\mathrm{cm} / \mathrm{s}, \mathrm{v}_{\mathrm{u}}(\mathrm{oxy})=3.65 \mathrm{~cm} / \mathrm{s}, \operatorname{dil}_{\mathrm{Ar}}=85.3 \%\right)$.

Figure 3-16. Normalized histograms of curvature (a), arc length (b), and diameter (c) and arc length versus diameter scatter plot (d) of amorphous carbon material..... 46

Figure 3-17. Normalized histograms of curvature (a), arc length (b), and diameter (c) and arc length versus diameter scatter plot (d) of condensable material collected at 
$\mathrm{h}=72 \mathrm{~mm}$ in flame $\# 11\left(\mathrm{p}=40\right.$ Torr, $\mathrm{v}_{\mathrm{u}}($ fuel $/ \mathrm{Ar})=820 \mathrm{~cm} / \mathrm{s}, \mathrm{v}_{\mathrm{u}}(\mathrm{oxy})=3.65 \mathrm{~cm} / \mathrm{s}$, $\operatorname{dil}_{\mathrm{Ar}}=85.3 \%$ ).

Figure 3-18. Normalized histograms of curvature (a), arc length (b), and diameter (c) and arc length versus diameter scatter plot (d) of condensable material collected at $\mathrm{h}=74 \mathrm{~mm}$ in flame $\# 11$ ( $\mathrm{p}=40$ Torr, $\mathrm{v}_{\mathrm{u}}(\mathrm{fuel} / \mathrm{Ar})=820 \mathrm{~cm} / \mathrm{s}, \mathrm{v}_{\mathrm{u}}(\mathrm{oxy})=3.65 \mathrm{~cm} / \mathrm{s}$, $\left.\operatorname{dil}_{\mathrm{Ar}}=85.3 \%\right)$.

Figure 3-19. Normalized histograms of curvature (a), arc length (b), and diameter (c) and arc length versus diameter scatter plot (d) of condensable material collected at $\mathrm{h}=75 \mathrm{~mm}$ in flame \#11 ( $\mathrm{p}=40$ Torr, $\mathrm{v}_{\mathrm{u}}(\mathrm{fuel} / \mathrm{Ar})=820 \mathrm{~cm} / \mathrm{s}, \mathrm{v}_{\mathrm{u}}(\mathrm{oxy})=3.65 \mathrm{~cm} / \mathrm{s}$, $\left.\operatorname{dil}_{\mathrm{Ar}}=85.3 \%\right)$.

Figure 3-20. Normalized histograms of curvature (a), arc length (b), and diameter (c) and arc length versus diameter scatter plot (d) of condensable material collected at $\mathrm{h}=77 \mathrm{~mm}$ in flame $\# 11$ ( $\mathrm{p}=40$ Torr, $\mathrm{v}_{\mathrm{u}}(\mathrm{fuel} / \mathrm{Ar})=820 \mathrm{~cm} / \mathrm{s}, \mathrm{v}_{\mathrm{u}}(\mathrm{oxy})=3.65 \mathrm{~cm} / \mathrm{s}$, $\operatorname{dil}_{\mathrm{Ar}}=85.3 \%$ ). 48

Figure 3-21. Curvature comparison for flame $\# 11\left(\mathrm{p}=40 \mathrm{Torr}, \mathrm{v}_{\mathrm{u}}(\mathrm{fuel} / \mathrm{Ar})=820 \mathrm{~cm} / \mathrm{s}\right.$, $\left.\mathrm{v}_{\mathrm{u}}(\mathrm{oxy})=3.65 \mathrm{~cm} / \mathrm{s}, \operatorname{dil}_{\mathrm{Ar}}=85.3 \%\right)$.

Figure 3-22. HRTEM image of fullerenic nanostructures in condensable material collected at $\mathrm{h}=61 \mathrm{~mm}$ in flame $\# 13\left(\mathrm{p}=12 \mathrm{Torr}, \mathrm{v}_{\mathrm{u}}(\right.$ fuel $/ \mathrm{Ar})=820 \mathrm{~cm} / \mathrm{s}$, $\left.\mathrm{v}_{\mathrm{u}}(\mathrm{Oxy})=3.65 \mathrm{~cm} / \mathrm{s}, \mathrm{dil}_{\mathrm{Ar}}=65.0 \%\right)$.

Figure 3-23. Quantity of fullerenes $\mathrm{C}_{60}$ and $\mathrm{C}_{70}$ in the condensable material from different heights above burner in different turbulent diffusion flames. 55

Figure 3-24. Concentration of fullerenes $\mathrm{C}_{60}$ and $\mathrm{C}_{70}$ in the cold probed gas from different heights above burner in different turbulent diffusion flames.

Figure 3-25. Mass ratio of $\mathrm{C}_{60}$ to $\mathrm{C}_{70}$ in the condensable material from different heights above burner in different turbulent diffusion flames.

Figure 4-1. Premixed flame chamber with burner, top plate, secondary ring injector, and flame sampling probe (from Grieco [37]).

Figure 4-2. Premixed flame burner with top view (from Grieco [37])............................... 64

Figure 4-3. Top view of large (a) and small (b) secondary ring injectors ..........................65

Figure 4-4. Top section of the premixed flame chamber......................................................65

Figure 4-5. Concentration of $\mathrm{C}_{60}$ and $\mathrm{C}_{70}$ in a premixed benzene flame ( $p=40$ torr, $\varphi=$ 2.4 , velocity $=25 \mathrm{~cm} / \mathrm{s}$, dilution $=10 \%$ )

Figure 4-6. Concentration of soot in a premixed benzene flame ( $p=40$ torr, $\varphi=2.4$, velocity $=25 \mathrm{~cm} / \mathrm{s}$, dilution $=10 \%$ )

Figure 4-7. Concentration of fullerenes in a premixed benzene flame ( $p=40$ torr, $\varphi=2.4$, velocity $=25 \mathrm{~cm} / \mathrm{s}$, dilution $=10 \%$ ) $[89]$ 
Figure 4-8. Concentration of DCM soluble material ( $\square$ ) and DCM insoluble material ( $\square$ ) in a premixed benzene flame $(p=40$ torr, $\varphi=2.4$, velocity $=25 \mathrm{~cm} / \mathrm{s}$, dilution $=10 \%)[89]$ 70

Figure 4-9. Flame gas concentration of $\mathrm{C}_{60}$ in McKinnon flame and three two-stage flames with benzene as secondary fuel. 76

Figure 4-10. Flame gas concentration of $\mathrm{C}_{60}$ in McKinnon flame and three two-stage flames with acetylene as secondary fuel. 76

Figure 4-11. HPLC chromatogram of soot extract from two-stage flame \#3 at $60 \mathrm{~mm}$ HAB. 78

Figure 4-12. HPLC chromatogram of soot extract from McKinnon flame at $50 \mathrm{~mm}$ HAB. 78

Figure 4-13. HPLC chromatogram of soot extract from McKinnon flame at 5.8 mm HAB. 79

Figure 5-1. Schematic of equipment for study of fullerene-soot reaction. 84

Figure 5-2. Fullerene-soot reaction rate plot - Day one data ( $\square$ ) with regression (longdashed line), Day two data $(\mathbf{\Delta})$ with regression (short-dashed line), and full regression (solid line) with equation. 88

Figure 5-3. Predicted consumption rate of fullerenes by soot in the McKinnon flame using fullerene and soot data from Grieco's study [89]. 91

Figure 5-4. Predicted consumption rate of fullerenes by soot in the McKinnon flame using fullerene data from the present study 92

Figure 6-1. HRTEM images of particle from pure carbon black sample. .98

Figure 6-2. HRTEM images of particle from post-extraction tethered fullerene sample with user-entered dashes to identify fullerene-type structures. 99

Figure 6-3. Fullerene-type structure diameter distribution data on an absolute basis. ... 100

Figure 7-1. Schematic of commercial carbon black plant 104

Figure 7-2. Senstivity of incremental fullerenic soot cost to soot yield and fuel price (reactor pressure $=40$ Torr and pump lifetime $=10$ years)

Figure 7-3. Senstivity of incremental fullerenic soot cost to reactor pressure and pump lifetime (soot yield $=3 \%$ and fuel price $=\$ 1.50$ per gallon)

Figure 7-4. Senstivity of incremental fullerenic soot cost to reactor pressure and pump lifetime (soot yield $=20 \%$ and fuel price $=\$ 0.50$ per gallon) 


\section{List of Tables}

Table 3-1. Laminar diffusion flame parameters ............................................................. 40

Table 3-2. Turbulent diffusion flame parameters ............................................................. 55

Table 4-1. Two-stage flame parameters.............................................................................. 74

Table 6-1. Fullerene-type structure linear concentration data. ......................................... 99

Table 7-1. Major parameters for Donnet's carbon black production plant....................... 105

Table 7-2. Base case incremental costs for fullerenic soot production.............................. 110 


\section{Introduction}

The family of compounds known as fullerenes consists primarily of pure carbon molecules. Each molecule contains an even number of carbon atoms between 36 and several thousand. Since fullerenes were first discovered, significant research has been performed to investigate their formation methods and potential applications. There has also been significant work to determine the mechanisms and reaction pathways that facilitate fullerene formation. Many such mechanisms have been proposed, however, none have thus far been conclusively proven or disproven. Despite this, the synthesis of fullerenes and fullerenic nanostructures through combustion of hydrocarbon fuels has been of particular interest recently and has shown potential to become a feasible method for commercial fullerene production.

\section{$1.1 \quad$ Background}

Fullerenes, discovered in 1985 [1], are the third form of pure carbon material behind graphite and diamond. They are made up entirely of $\mathrm{sp}^{2}$-bonded carbon atoms which are arranged in pentagonal and hexagonal rings. The pentagonal rings cause strain within the structure which is alleviated by out-of-plane deformation or curvature at each pentagon. This curvature in the carbon structure leads to the hollow cage configuration characteristic of fullerene molecules. The most stable configuration of rings obeys the Isolated Pentagon Rule: no two pentagons in a stable fullerene can be adjacent. The number of pentagons in a molecule is fixed at twelve as this provides sufficient curvature to form a closed shell. However, the number of hexagons varies with the total number of carbon atoms according to Equation 1-1:

$$
\text { hexagons }=\frac{\text { carbon atoms }-20}{2}
$$

A $_{60}$ molecule, for example, contains 60 carbon atoms arranged symmetrically about a sphere into 12 pentagons and 20 hexagons. The arrangement of the pentagons on the surface determines the final cage structure. Evenly distributed pentagons will result in a sphere, as is the case for the most common isomer of $\mathrm{C}_{60}$.

The two most abundant and well-known fullerenes are $\mathrm{C}_{60}$ and $\mathrm{C}_{70}$. $\mathrm{C}_{60}$ is also known as Buckminsterfullerene, named for R. Buckminster Fuller, who designed the 
geodesic domes that resemble the $\mathrm{C}_{60}$ molecule [1]. Only one configuration for each of these two molecules obeys the Isolated Pentagon Rule. Being the most stable, these configurations are the most common and are shown in Figure 1-1.

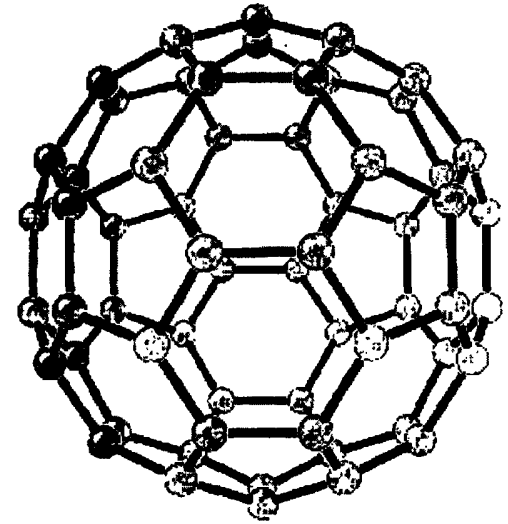

(a)

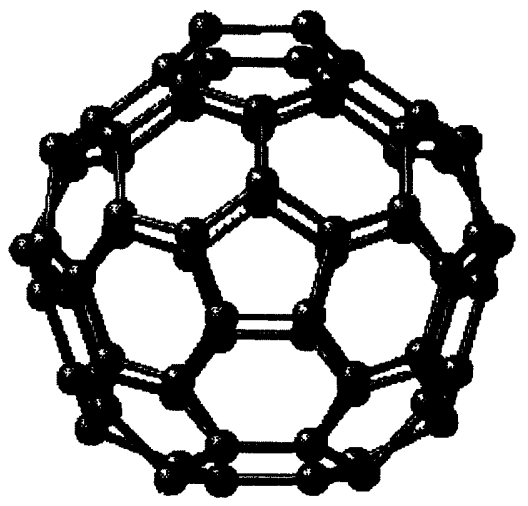

(b)

Figure 1-1. Images of $C_{60}(a)$ and $C_{70}(b)$.

Fullerenes as small as $\mathrm{C}_{20}$ and as large as $\mathrm{C}_{>300}$ have also been identified [2-4]. Those that consist of less than 300 carbon atoms are generally known as 'buckyballs' and are restricted to single-shell structures. Single metal atoms such as lanthanum or cerium can be trapped inside the cavity of these single-shell structures [5-8] creating a class of fullerenes known as endohedral fullerenes (or metallofullerenes). Giant fullerenes $\left(C_{>300}\right)$, however, may be single or multiple shelled. Multiple-shelled giant fullerenes are known as 'onions' as the adjacent multiple layers resemble those of an onion. Giant fullerene 'nanotubes' (or 'buckytubes') have also been identified [9]. These structures, which can be single- or multiple-shelled, consist of cylindrical walls composed entirely of hexagonal rings capped at each end by six pentagonal rings and the necessary hexagonal rings to close the structure. Nanotubes with diameters as small as $4 \AA$ have been observed [10-12] meaning that the fullerenic caps are smaller than $\mathrm{C}_{60}$ molecules, which have a diameter of $7 \AA$. Throughout the remainder of this work, to avoid confusion, buckyballs will be referred to simply as 'fullerenes', while onions and nanotubes will collectively be referred to as 'nanostructures'. 


\subsubsection{Formation of fullerenes and nanostructures}

Since their discovery in 1985 [1], there has been significant research into methods for synthesizing fullerenes. Kroto et al. [1] first identified fullerenes in carbon vapor from the laser ablation of graphite; Iijima used the same method to first observe $\mathrm{C}_{60}$ directly [13]. This method, however, resulted in microscopic quantities of fullerenes insufficient to provide samples for characterization and research. In 1990, fullerenes were produced in macroscopic quantities by striking an electric arc between two graphite electrodes and evaporating the graphite in a low-pressure ( $<100$ Torr), helium atmosphere [14]. This method produces fullerenes along with a carbonaceous soot, from which the fullerenes can be separated. Although the yield of fullerenes is highly dependent on operating conditions, chamber design, and experimental technique, typical yields from this method are $10 \%$ of the collected soot [15]. At higher pressures ( 500 Torr), this method has produced nanostructures and, using electrodes doped with metal, endohedral fullerenes [7]. A modified version of this graphite arc-vaporization method is the predominant method for supplying the current commercial demand for fullerenes (see Section 1.4) [15].

Other methods of fullerene synthesis include ion sputtering and electron beam evaporation of graphite [16], vaporization of graphite using highly concentrated solar heating $[17,18]$, resistive heating of graphite [19], laser ablation of graphite $[5,20]$, inductive heating of graphite [21], and carbon particle evaporation in a hybrid thermal plasma [22]. Several production methods using non-graphitic raw materials have also been studied and found to produce fullerenes. These methods include pyrolysis of naphthalene in an argon atmosphere at approximately $1000^{\circ} \mathrm{C}$ [23], the pyrolytic destruction of toluene in a radio frequency plasma [24], and laser-pyrolysis of benzene [25]. None of these methods, however, produce macroscopic quantities of fullerenes.

Similarly, fullerenic nanostructures can be generated under a range of conditions which were first identified in 1991 by lijima [9] in solid deposits on the cathode of a graphite arc-vaporization apparatus operating under a pressure of 100-200 Torr. By increasing the pressure to 500 Torr, Ebbesen et al. [26] found that they could produce large quantities of nanotubes. Iijima went on to discover single-shelled nanotubes in his 
arc-vaporization process [27], while Bethune et al. [28], among others, catalyzed nanotube production by using metal-doped graphite rods in arc-vaporization.

Separate from fullerenes, nanostructures have also been produced independently through arc-discharge vaporization [9-11], plasma-decomposition of benzene [29], pyrolysis of tripropylamine [12], and in a pyrolysis flame of carbon monoxide and hydrogen [30]. They have also been produced on large-scale by the catalytic decomposition of hydrocarbons at about $1200^{\circ} \mathrm{C}[31]$.

Fullerenes and nanostructures have both been discovered in the products formed from the combustion of organic fuels [32-38]. The combustion synthesis of fullerenes and fullerenic nanostructures is the major focus of this work and background studies are discussed in much greater detail in Sections 1.2 and 1.3.

In addition to synthetic methods of production, fullerenes have also been found in nature. Evidence has been gathered from several different sources to indicate that the formation of fullerenes is not solely a man-made occurrence. On Earth, fullerenes, primarily $\mathrm{C}_{60}$ and $\mathrm{C}_{70}$, have been extracted from two Cretaceous-Tertiary boundary sites in New Zealand and may be the result of extensive wildfires [39]. They have also been found in fulgurite, a glassy rock that forms when lightning strikes the ground [40], in coal samples found in China [41, 42], and in shungite, a carbon-rich Precambrian rock from Karelia, Russia [43]. Fullerenes were also found in residue from the Allende meteorite [44] and the 1.85-billion-year-old Sudbury meteorite impact structure in Ontario, Canada [45]. Previous work has shown the presence of fullerene-like structures in soot from regular candle flames and wood fires thereby suggesting that fullerenes may be formed in trace quantities in such flames [37]. There is also evidence of fullerenes in outer space as they were observed in the impact craters of NASA's Long Duration Exposure Facility (LDEF) spacecraft [46], and are believed to be present in interstellar material $[47,48]$, where they serve as carriers for extraterrestrial noble gases [49-51]. These observations of fullerenes indicate that they are formed in highly energetic, natural processes as well as by synthetic methods [37]. 


\subsubsection{Fullerene properties and reactivity}

Fullerenes have attracted enormous interest because of their stability, high symmetry, and other properties which have important practical applications. They have been found to have a number of unique physical and chemical properties that makes them ideally suited for wide array of applications and specific reactions (which are discussed in the next section). These properties stem primarily from the three-dimensional closedshell configuration that occurs with the inclusion of 12 carbon pentagons into a molecule.

Most significantly, fullerenes such as $\mathrm{C}_{50}, \mathrm{C}_{60}$, and $\mathrm{C}_{70}$ have been identified as 'magic number' carbon clusters meaning that their ionization potentials are greater than other carbon clusters immediately around them [52-54] and also that the reaction rates of their ions with metallocenes are anomalously slow compared to those of other large clusters [52]. Additionally, thermochemical calculations have shown that the heats of formation for $\mathrm{C}_{60}$ and $\mathrm{C}_{70}$ may be significantly greater than other carbon molecules of similar size [55] indicating that an enormous amount of energy is being stored in the delocalized $\pi$-bond system. The enthalpies of formation for many fullerene molecules have also been calculated by various investigators resulting a fairly large database of thermodynamic values $[56,57]$. These results indicate that fullerenes are more stable than neighboring clusters which would be expected from their spherical nature.

Fullerenes also exhibit an uncharacteristic behavior in that their solubility in organic solvents goes through a peak with respect to temperature. This solubility behavior is caused by an aggregation of fullerenes molecules in solution and by a change in the solid-phase at high-temperatures leading to a disordered solution. Although the peak temperature is variable for different solvents, this phenomenon has found lots of different applications in extraction and purification procedures [58].

In terms of more practical properties of both fullerenes and nanostructures, they have been found to have both superconductivity [59] and semiconductivity [60, 61], have a high affiliation for hydrogen storage [62], and posses the ability to assemble themselves into rods [63]. Additionally, fullerenes have the ability to trap other gases, metal atoms, or molecules inside their hollow cavities making them useful for transport and delivery [5-7]. It has also been discovered that the sublimation temperatures of different species of 
fullerenes are sufficiently different from each other and from soot to allow for the separation and purification of fullerenes via sublimation [64].

The chemistry of fullerenes has also been the focus of intense research over the past few years. While once thought to be relatively stable and chemically inert [65-67], it has now been shown that fullerenes do in fact react with a variety of other molecules under a range of conditions. In 1993, Taylor et al. found that they undergo reactions that are characteristic of alkenes and that fullerene derivatives formed from these reactions offered new directions for organic chemistry. Among other things, they found that fullerenes, $\mathrm{C}_{60}$ in particular, undergo addition reactions, nucleophilic and electrophilic substitutions, and even polymerization [68].

Suzuki et al. discovered that phenyl rings could be added to fullerenes to make fulleroids ranging from $\mathrm{C}_{61}$ to $\mathrm{C}_{66}$ [69]. Fullerene-phenyl compounds in the form of $\mathrm{C}_{60^{-}}$ $\mathrm{C}_{5} \mathrm{H}_{6}$ have also been identified as products from flames [70] as well as from a DielsAlder reaction [71].

It has also been shown that fullerenes are subject to attack by benzyl radicals resulting in both radical and non-radical adducts [72]. The radical products were found to have extraordinary stability as a result of the steric protection of surface radical sites by surrounding benzyl substituents while non-radical products were found to have up to 34 methyl groups attached to the fullerene surface.

It is these radical and Diels-Alder reactions that have provided an explanation for the chemical interaction between carbon black, which is mature combustion-generated soot material, and diene rubber during mixing $[73,74]$. The observation of fullerene-like sites on the surface of the carbon black accounts for the addition of polymer rubber chains to the surface of carbon black.

Fullerenes have been identified in countless other reaction pathways and kinetic systems involving a wide range of industries. However, the diverse nature of these reactions precludes a comprehensive review of all fullerene properties and reaction systems. The reactions discussed here form a small basis set of this database and are meant to provide a general background to explain the vast number of fullerene applications have been proposed and are currently being researched. 


\subsubsection{Applications}

Due to their diverse chemistry and properties (as discussed in the previous section), potential applications for both fullerenes and fullerenic nanostructures have been identified in a wide range of fields. These applications together form a highly diversified technology portfolio [75].

It has been found that fullerenes are versatile building blocks for making a variety of molecular and supramolecular structures such as inorganic superconductors, organic polymers, and biologically active molecules [63]. Potential applications for fullerenes include: use as optical limiters and photoconductors, for film growth and patterning, as catalysts for organic reactions, for hydrogen and energy storage, in lubrications and separations [76], and in extractions and purifications [58]. More specifically, biological applications have been proposed including use in AIDS and cancer treatment [77] and to make immunological antibodies [78], its electrical properties make them good candidates to be superconductors [59] and electronic transistors [79, 80], and it has been used as a precursor molecule for nanocrystalline diamond film growth through chemical vapor deposition [81]. On a commercial basis, The Goodyear Tire and Rubber Company has obtained a patent for the use of fullerenes and fullerenic soot in the production of curable and vulcanized rubber indicating that the rubber made from these raw materials is superior to that manufactured from ordinary carbon black [82].

Nanostructure applications include use in construction materials, heavy-duty shock absorbers, heat sinks, and chemical probes [75]. Nanotubes have also been identified as a potential hydrogen storage medium for use in fuel cells [62], a probable successor to silicon in making faster microprocessors $[60,61]$, and a superior sorbent for the removal of dioxin [83].

Despite the multitude of potential applications, extensive research has been impeded by the prohibitive cost of producing and isolating fullerenes. One of the objectives of this work (as discussed in Section 2) is to identify a less expensive production method for fullerenes and fullerenic soot in an attempt to lower the commercial price and spur the use of identified applications as well as research into new applications. 


\subsection{Fullerene formation in flames}

Since fullerenes were first positively identified as products of flames [35], fullerene-forming combustions systems have been the subjects of quite extensive study. In an attempt to realize the optimal conditions for fullerene yield, numerous flames have been characterized and documented with respect to fullerenic content. In conjunction, a vast effort has been made to predict and verify the mechanisms of fullerene formation to better understand and design desirable flame conditions. The overall goal of this combustion work is to someday identify a combustion system that would be suitable for the large-scale production of fullerenes and fullerenic soot.

\subsubsection{Methods of production}

The existence of fullerenes in flames was first suggested in 1986 [67] and fullerene ions were detected in flat premixed acetylene-oxygen and benzene-oxygen flames in 1987 [84]. It was not until 1991, however, that fullerenes were first identified in material recovered from flames. Fullerenes were found in soot produced from lowpressure premixed benzene/oxygen/argon flames [35, 85-87]. This work produced macroscopic quantities of $\mathrm{C}_{60}$ and $\mathrm{C}_{70}$ and identified combustion as a feasible process for the synthesis of fullerenes. Results from this work indicated that the fullerenic yield was highly dependent on temperature, pressure, residence time, and fuel to oxygen ratio. Variables to control these parameters, such as chamber pressure, dilution, and cold gas fuel velocity, were adjusted over wide ranges to produce various flames and characterize fullerene production. In these studies, the yield of $\mathrm{C}_{60}$ ranged from $0.003 \%$ to $9 \%$ (by mass) of the collected soot and $0.001 \%$ to $0.7 \%$ (by mass) of total carbon fed. Additionally, fullerenes as large as $\mathrm{C}_{116}$ have been seen in similar flame experiments performed by others [88].

The formation of fullerenes has also been studied in flames that arise from the combustion of other organic fuels. These include naphthalene [32], butadiene [32], toluene [33], and organics with halogen additives [34]. Of the pure organic fuels, it has been shown that benzene produces the greatest amounts of fullerenes [32, 33], but that production may be enhanced by the addition of a chlorine additive [34]. 
Recently, both premixed and diffusion laminar benzene/oxygen/argon flames have been researched in much greater detail. Premixed flame studies indicate two distinct regions of fullerene formation and consumption with increasing height above burner [37, 89]. Results also showed that fullerene formation has only marginal dependence on pressure and dilution but is significantly correlated to gas velocity [86]. The effect of velocity is an indirect relationship as gas velocity affects temperature in the flame, and temperature affects fullerene formation. Diffusion flame studies have shown that fullerene formation reaches a maximum at the stoichiometric surface of a flame and peaks with increasing argon dilution. Results have indicated that fullerene formation is strongly correlated to flame length and temperature. These two parameters are both correlated to chamber pressure, argon dilution, and cold gas fuel velocity. Typical fullerene yields of $0.5 \%$ (by mass) of total carbon fed were observed but can possibly be higher $[90,91]$.

\subsubsection{Fullerene formation mechanisms}

Although the mechanism for fullerene formation in combustion systems has not been definitively described and is still under much debate, many possible pathways have been proposed. These pathways all involve reactions or rearrangements of carbon molecules believed to be present in flames and can be categorized as gas-phase or solidphase mechanisms. The proposed mechanisms, it should be noted, do not describe specific reactions among particular molecules but instead provide broad conceptualizations of the types of reactions that may be occurring to produce fullerenes.

The formation of fullerenes in the gas-phase is thought to occur mainly through molecular weight growth reactions similar to those seen in the formation of polycyclic aromatic hydrocarbons (PAH) and soot. These reactions can involve either the step-wise addition of low-molecular weight species, such as acetylene, to radical sites on larger aromatic compounds [92], or the coagulation of two larger specific PAH molecules and/or carbon clusters [93]. Similar to the HACA mechanism proposed for PAH formation [94], the step-wise addition mechanism for fullerenes would proceed through abstraction of an $\mathrm{H}$-atom from an aromatic molecule. This would be followed by $\mathrm{C}_{2} \mathrm{H}_{2}$ addition and cyclization leading to ring closure. Eventually, once the molecule had grown 
large enough, this mechanism would lead to cage closure and the formation of a fullerene molecule [92]. The reactive coagulation mechanism would involve two or more larger aromatic molecules (and most likely curved, indicating the presence of pentagonal rings) coming together with hydrogen elimination to directly form fullerenes [93]. It is thought that both of these mechanisms contribute to fullerene production in flames [92], but has been suggested that coagulation is the predominant method [95].

Another gas-phase assembly mechanism that has been proposed involves the zippering up of one or two carbon molecules followed by intramolecular rearrangement to form a stable fullerene species. In one method, two PAH molecules (most likely planar) align their peripheries so they can be easily connected as if the molecules were being zipped together [32]. This zippering forms a closed-shell structure that would necessarily have the correct number of pentagons and would then rearrange itself to form the most stable isomer of fullerene. In a similar proposed method, a large ribbon-shaped molecule, such as polyacetylene or a PAH already containing five-membered rings, would curl up and zip to itself [96]. Once zipped up, the molecule could then undergo intramolecular rearrangement, reaction with benzene molecules, and/or hydrogen elimination if necessary. While it is believed that the first zippering mechanism could be plausible in flames, the second is considered unlikely [93].

It has also been proposed that fullerenes are formed in flames in condensed solidphase mechanisms. They have been observed as products of internal rearrangement reactions occurring on the surface of carbon black particles subjected to heat treatment and electron beam irradiation under an inert atmosphere [97]. This study indicates that pentagonal defects are created in hexagonal sheets of graphite and suggests that a similar process could be occurring on soot particle surfaces on flames. A second possibility for solid-phase formation is the occurrence of heterogenous reactions analogous to the gasphase reactions discussed above. In such a scenario, fullerene precursor molecules could adsorb (physically or chemically) to a soot particle, undergo the necessary reactions for cage closure, and then be released back into the vapor phase [98]. Alternatively, a precursor molecule need not adsorb to a soot particle as the fullerene forming reactions could occur with a PAH or carbon molecule already present on the surface. 
It is important to note that all of the mechanisms discussed here could be contributing to observed fullerene concentrations. The mechanisms are not mutually exclusive and each may be predominant at different residence times and different flame conditions, as suggested by Grieco et al. [89]. It appears that consumption of fullerenes by soot also plays a role in fullerene production suggesting that the observed fullerene yields are actually small differences between large formation and consumption reactions [89]. Trying to separate the effects of these mechanisms, and of the consumption of fullerenes, is one of the focuses of this work (Chapters 2 and 5).

\subsection{Nanostructure formation in flames}

The formation of fullerenic nanostructures in combustion was first identified in 1994 [38] and has since been studied in both premixed and diffusion laminar benzene/oxygen/argon flames $[36,37,90,91]$. These studies all used high resolution transmission electron microscopy (HRTEM) images to perform a qualitative analysis of the evolution of nanostructures in flames while Grieco [37, 99] also performed a quantitative analysis. It was observed that nanostructures are formed in fullerene-forming flames and that the amount of closed-shell structures and fullerenic material increases with residence time, or distance from the burner. These HRTEM images show that soot becomes more ordered farther from the burner [37, 90, 91, 99]. The premixed study also found that nanostructures require a much longer residence time to form than do fullerenes [37]. The diffusion flame analysis showed that nanostructures are formed higher than the

point of maximum fullerene production where soot and fullerenes are being consumed by oxidation and the flame is the hottest $[90,91]$. Additionally, structures similar to those observed in fullerene-forming flames have also been found in commercially-produced carbon black [100, 101].

Although considerable interest has been given to the formation of nanostructures in the methods described here and in Section 1.1.1, there has only been limited work into formation mechanisms and even less into mechanisms in combustion systems. The mechanisms that have been proposed can again be split into gas-phase and solid-phase mechanisms. The gas-phase mechanisms are similar to those of fullerenes and involve step-wise addition that lead to tube lengthening instead of cage closure [102]. The solid- 
phase mechanisms involves a graphitic sheet (or sheets) of carbon rolling up to form cylinders that can be capped to form nanotubes [37]. Structural rearrangement of such tubes or other graphitic sheets can also be used to explain the formation of fullerene onions and other nanostructures [37]. A plethora of nanotubes have also been found in flames with metal additives suggesting a catalyzed formation process [103, 104]. These proposed mechanisms, however, are still highly speculative and none have been proven or disproven.

\subsection{Commercial production of fullerenes}

The prevalence of so many useful applications for fullerenes and the large potential market for them has spurred an acute interest in the large-scale commercial production of both fullerenes and fullerenic soot. Although fullerenes still remain prohibitively expensive for the vast majority of the proposed applications and even for basic research, the cost has dropped quite significantly over the past decade from $\$ 10,000$ per gram in 1991 to around $\$ 50$ per gram in 2000 for $C_{60}$ [105] and up to $\$ 55,000$ per gram for larger fullerenes in 1999 [106]. Currently, the price of $99.9 \%$ pure $\mathrm{C}_{60}$ is $\$ 20$ per gram [106]. It is estimated that current $C_{60}$ prices may be low enough for expensive applications, such as those in the pharmaceutical industry, but for cheaper uses, such as electronics and polymers, the price would need to drop to at least $10 \notin$ per gram and maybe as low as $1 \phi$ per gram $[105,107]$. Using the current predominant method for commercial production of fullerenes [15], it has been estimated that the fullerene cost could drop no lower than 25\& per gram [108], and probably not even that low [109]. Fullerenic soot, on the other hand, which would need to compete with the current carbon black cost of $35 \notin$ per pound ( $77 \notin$ per $\mathrm{kg}$ ), currently has only severely limited commercial production and costs around $\$ 1500$ per pound ( $\$ 3300$ per $\mathrm{kg}$ ) [106]. The current production technology is not expected to provide a significant drop in these prices in the near future.

Given the disparity between the estimated lowest future fullerene price using graphite-arc vaporization and the necessary price ceiling to make fullerenes widely competitive, it is natural that other methods of fullerene synthesis be explored and studied in detail for possible commercial scale-up. One method that has emerged as the most 
likely to replace arc vaporization in commercial production is combustion synthesis. Combustion offers several advantages over carbon-arc vaporization: the raw material costs are much lower since organic fuels are cheaper than the pure graphite rods used in arc vaporization; operating costs are significantly reduced as vaporization requires a significant energy input; and combustion systems can be easily scaled up for mass production whereas arc vaporization cannot due to limitations on the diameter of the graphite rod. Additionally, a comparison study of five different fullerene synthesis methods concluded that sooting hydrocarbon flames would be the most suitable for industrial production [110]. Preliminary analysis of a commercial fullerene synthesis plant utilizing combustion indicates that fullerene costs could drop as low as 7-8 $\mathrm{c}$ per gram [111], which would make fullerenes affordable for a wide variety of applications. Lastly, sooting flames have an enormous advantage in the production of fullerenic soot (soot containing fullerenes prior to their extraction) given that the whole purpose is to make soot. A slight modification to the operating conditions of current carbon black production technology, which uses combustion to generate vast amounts of soot, may be sufficient to commercially produce fullerenic soot.

Before combustion synthesis can be used successfully, however, it is necessary to identify the optimal operating conditions for fullerene and soot production from both a yield and economic point-of-view. These conditions can only be identified through a more detailed characterization and study of potential combustion systems coupled with a greater understanding of the formation mechanism to help design a suitable flame. Only once such a flame condition (and corresponding maximum fullerene yield) is determined can combustion be viewed as a practical alternative to graphite arc-vaporization. It is with this overall goal in mind that this study was performed. 


\section{Objectives}

The main objectives of this work were to investigate the yield of fullerenes and fullerenic nanostructures in various combustion systems involving premixed and diffusion benzene/oxygen/argon flames at low-pressure. In each system, operating conditions such as chamber pressure, argon dilution, cold fuel velocity, and fuel flowrates were varied to gain a better understanding of the flame characteristics. In addition, the formation and consumption mechanisms of fullerenes in flames were investigated through heat-treatment experiments to effect a better understanding of the response of the yield to changes in operating conditions. An HRTEM study of fullerenes tethered to soot material was also performed to positively identify round soot structures seen in images as fullerenes rather than false indications of fullerenes. Lastly, the commercial production of fullerenic soot was considered. The objectives are outlined as follows:

1. Investigate fullerene and nanostructure formation in diffusion flames under various conditions including laminar flow conditions and turbulent conditions with high fuel and cold gas feed rates.

2. Investigate fullerene and nanostructure formation in premixed flames under various conditions including those previously studied (for comparison) and the decoupling of the energy and reactant supplies for the flame through the use of two-stage flames.

3. Provide an understanding of the mechanism of fullerene formation and consumption through the study of the reaction of fullerenes. with soot.

4. Positively identify fullerene structures in HRTEM images by tethering fullerenes to soot to verify that fullerenes are indeed being observed.

5. Investigate the scale-up of a combustion synthesis process for the commercial production of fullerenic soot. 
Diffusion flame studies

To accomplish the objectives for diffusion flames outlined in Section 2, a number of experiments were performed in a diffusion flame combustion chamber and quantitative and qualitative analyses were performed on collected material. These experiments involved the study of fullerene and nanostructure formation in both low-flow laminar flames (referred to as 'laminar' in this section) and high-flow turbulent-like flames (referred to as 'turbulent'). In general, laminar flames were found to be quite interesting in terms of fullerenic production whereas the turbulent flames were not nearly as promising.

\subsection{Apparatus and techniques}

Producing fullerenes through combustion involves burning a fuel under sooting conditions in a combustion chamber and collecting the resulting condensable material, which includes polycyclic aromatic hydrocarbons, fullerenes, and of course soot. The fullerenes can then be extracted with toluene and separated from the soot, which is toluene insoluble, by filtration. A sample of the filtered liquid is then injected into a high performance liquid chromatography (HPLC) instrument to determine the fullerene concentration. Additionally, a sample of the condensed material before or after extraction can be placed on an electron microscope grid for examination by HRTEM, which is used to identify and characterize soot structure and nanostructures.

\subsubsection{Flame chamber}

The diffusion flame chamber, shown schematically in Figure 3-1, consists of a burner and fuel injection system mounted in the bottom of a cylindrical chamber housing. Two chamber extensions can be connected to the top of the housing and a plate seals the chamber at the top. The chamber has several view and auxiliary ports allowing viewing from multiple heights and angles, and allowing the quartz sampling probe, which is used for collecting condensable material, to be inserted in a number of locations including the top. The two-headed arrows in Figure 3-1 indicate the adjustable parts of the chamber.

The chamber is evacuated with a vane-type Mannesmann-Demag rotary vacuum pump (Model WPSO 53) with a capacity of 80 liters per second. The pressure is 
maintained by bleeding air into the vacuum system and is monitored by an MKS Instruments Baratron capacitance manometer (Model 690).

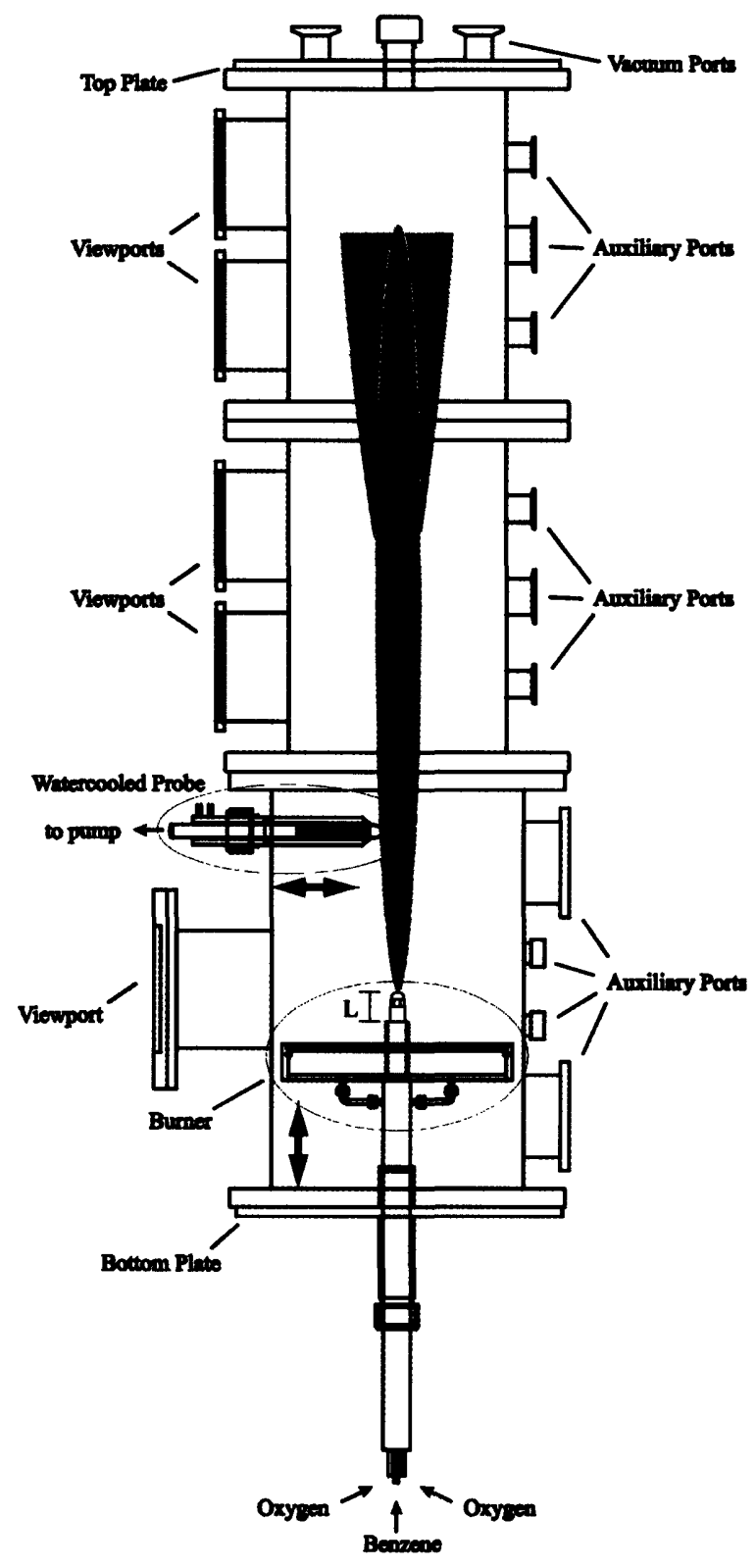

Figure 3-1. Diffusion flame chamber with burner and flame sampling probe.

\subsubsection{Flame burners}

Two different burners were used in the diffusion flame chamber. The first, shown in Figure 3-2, was used for low-flow flames while the second, discussed later in this section, could accommodate liquid fuel and was used for high-flow flames. 


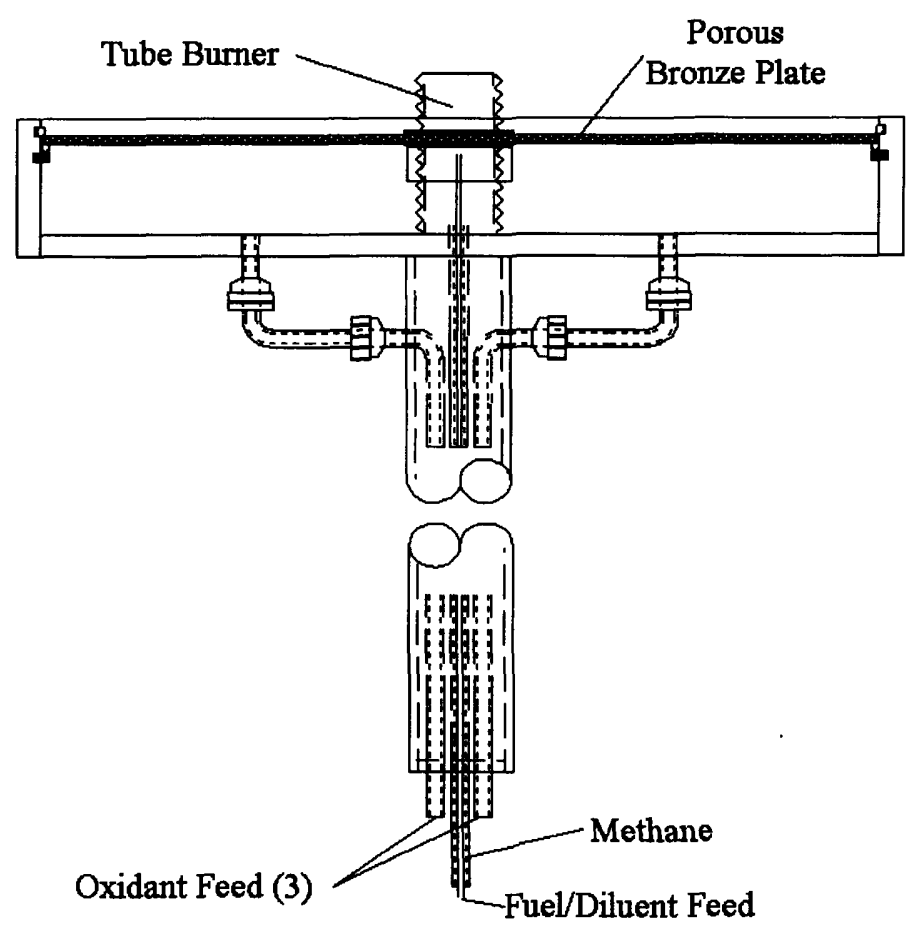

Figure 3-2. Low-flow diffusion flame burner.

The burner shown in Figure 3-2 consists of a stainless steel hollow disc (12.7 in. OD $\times 2.0$ in. thickness) that is attached axially to the top of a stainless steel tube $\left(1 \frac{1}{2} \mathrm{in}\right.$. OD $\times 24$ in.) and welded on the bottom. The tube supports the burner inside the chamber and passes through a port in the bottom plate. The tube can be moved to allow for the vertical adjustment of the burner.

A stainless steel tube burner ( $1 \frac{1}{2} \mathrm{in}$. ID) is also attached axially inside the disc and is encircled by a removable bronze porous plate that extends to the disc edge. O-rings are used to seal the burner to the porous plate and the plate to the disc. The burner is threaded allowing the use of burner caps with varying diameters. Several auxiliary burner caps were built with varying inner diameters. A diagram showing dimensions of several of the caps is shown in Figure 3-3. In addition to those shown, a cap with an outlet diameter of $3 \mathrm{~mm}$ was also fabricated.

Four smaller tubes ( $1 / 4 \mathrm{in}$. OD), located inside the larger tube, are the feedthroughs for the fuel, oxygen, and diluent. The middle tube feeds directly into the tube burner while the other three, through with oxygen is fed, feed into the disc and through the porous plate. The middle of the four tubes surrounds a smaller tube (1/8 in. OD) through 
which the fuel and diluent flow. Methane is fed through the annular region between the two tubes and is used as an ignition fuel for the flame. The ignition source for a flame is an electrical spark between the top of the burner and an igniting rod.
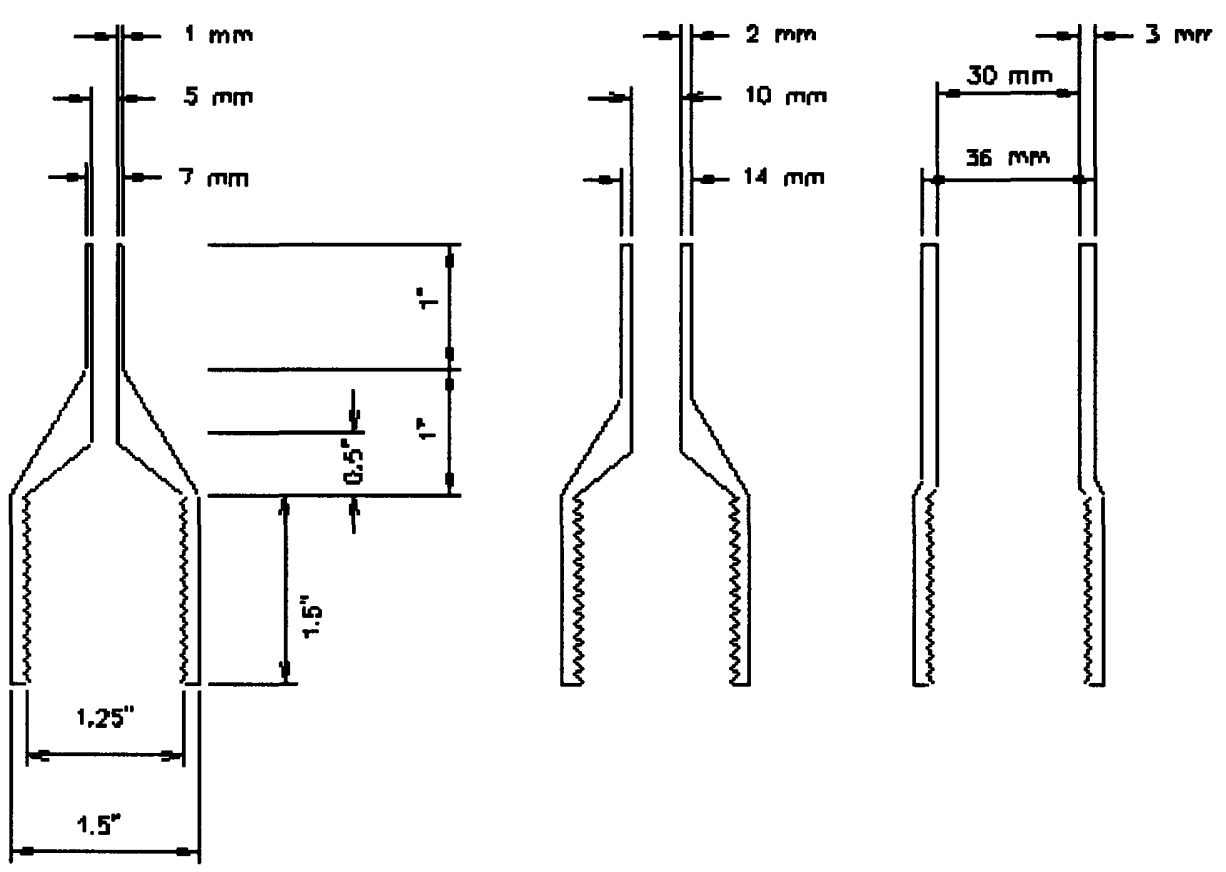

Figure 3-3. Burner caps with varying outlet diameters.

The second burner, used for turbulent flames in the diffusion chamber, is shown three different ways in Figure 3-4. Part (a) shows a side-view, part (b) shows a top-view and part (c) shows a cross-section to illustrate how the burner nozzle fits into the burner.

The burner consists of a stainless steel tube ( $1 \frac{1}{2} \mathrm{in.}$ OD x $30 \mathrm{in}$.) and contains seven feedthrough tubes instead of four as in the previous burner. Oxygen is fed to the four smaller tubes $(1 / 4 \mathrm{in}$. OD) on the periphery and is rerouted outside the tube to allow for its diffusion into the fuel at the burner outlet. A fourth oxygen feed is used here to compensate for the absence of the porous bronze plate, which ensured an evenly distributed oxygen flow. The smaller tube along the axis of the burner is used for the methane starter fuel while the two larger tubes $(3 / 8$ in. OD) are used for the fuel and diluent feed. 


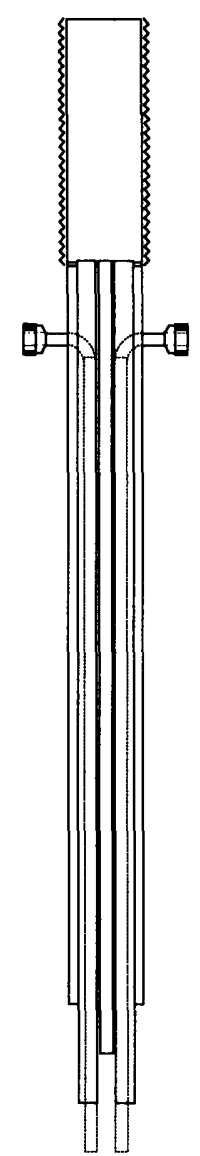

(a)

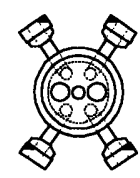

(b)

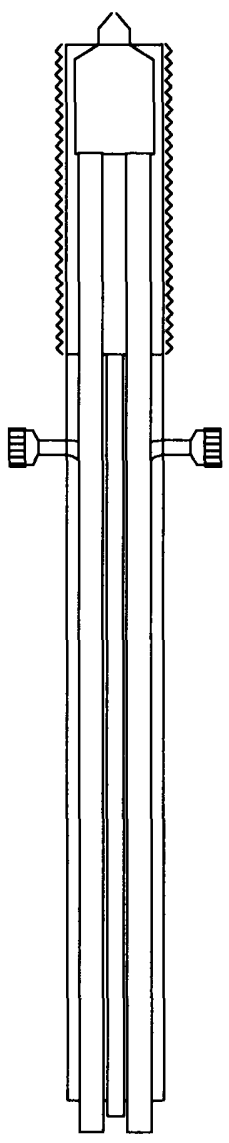

(c)

Figure 3-4. High-flow diffusion flame burner: side-view (a); top-view (b); and cut-away side-view with nozzle attached (c).

This burner allows for the use of an air-atomizing nozzle (Spraying Systems Company Model 1050 with Spray Cap Model 67147), which is shown mounted on the burner in Figure 3-4(c). Such a nozzle has two inlet ports and mixes liquid and vapor phases to produce an atomized spray. With this setup, it is possible to dilute the fuel, fed as a liquid to achieve turbulent flames, with vapor diluent.

To stabilize the nozzle and the flame, a burner cover, shown in Figure 3-5 was built. As can be seen, this cover consisted of a stainless steel base with a ceramic top and could be screwed onto the top of the burner. The large hole in the middle ( $5 / 8$ in. OD) fits exactly on top of the outlet of the nozzle and the sixteen smaller holes (1/16 in. OD) surrounding the center allow the methane to flow through to the top of the cover. 


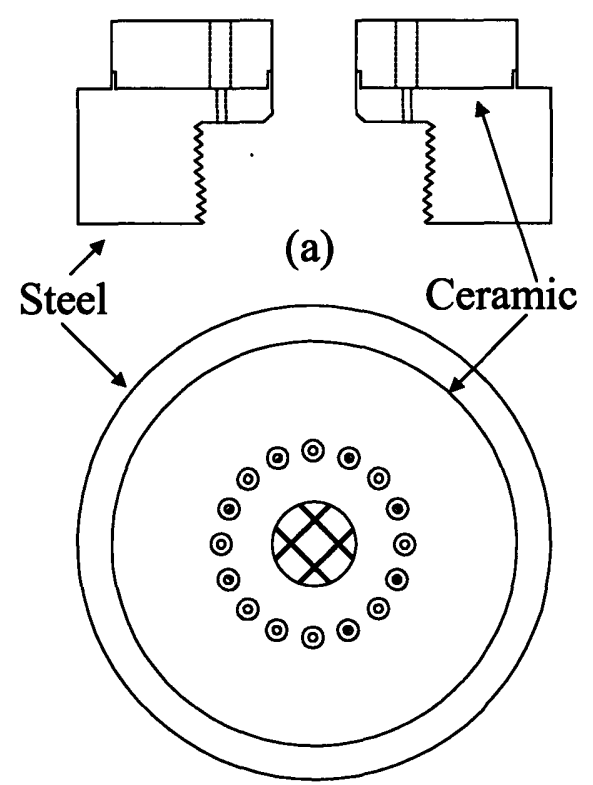

(b)

Figure 3-5. High-flow flame burner cover: side-view (a); and top-view (b).

The cover has four main effects: 1) it separates the methane flame from the benzene flame; 2) it protects the nozzle from the heat produced by the flame, which prevents flash evaporation of the fuel inside the nozzle; 3) it keeps the nozzle exactly in the center of the burner; and 4) it ensures the nozzle is always at the same height relative to the top of the burner. All four of these effects help in achieving a stable and consistent flame.

\subsubsection{Chamber sections}

The chamber consists of three sections. The top two sections are identical and removable so neither, one, or both may be used. If both are used, flames up to a length of 51 in. can be achieved. The bottom section (with the bottom plate and burner attached) is shown in Figure 3-6; the upper two sections are shown in Figure 3-7.

The bottom section consists of a cylindrical stainless steel chamber $\left(13^{1 / 4} \mathrm{in}\right.$. ID $x$ 24 in.) equipped with two end flanges, six auxiliary ports, and four viewports. The viewports are used for visual observation and are equipped with glass windows of 53/4 in. (1) and $4.0 \mathrm{in.} \mathrm{(3)} \mathrm{diameters.} \mathrm{The} \mathrm{three} \mathrm{4-in.} \mathrm{auxiliary} \mathrm{ports} \mathrm{are} \mathrm{sealed} \mathrm{with} \mathrm{stainless} \mathrm{steel}$ plates and house connections for pressure relief lines and a pressure transducer. The remaining three auxiliary ports are $1 \mathrm{in}$. and sealed, but provide access points for a 
sampling probe or other accessories. The base plate seals the bottom of the chamber and provides a mounting location for the burner.

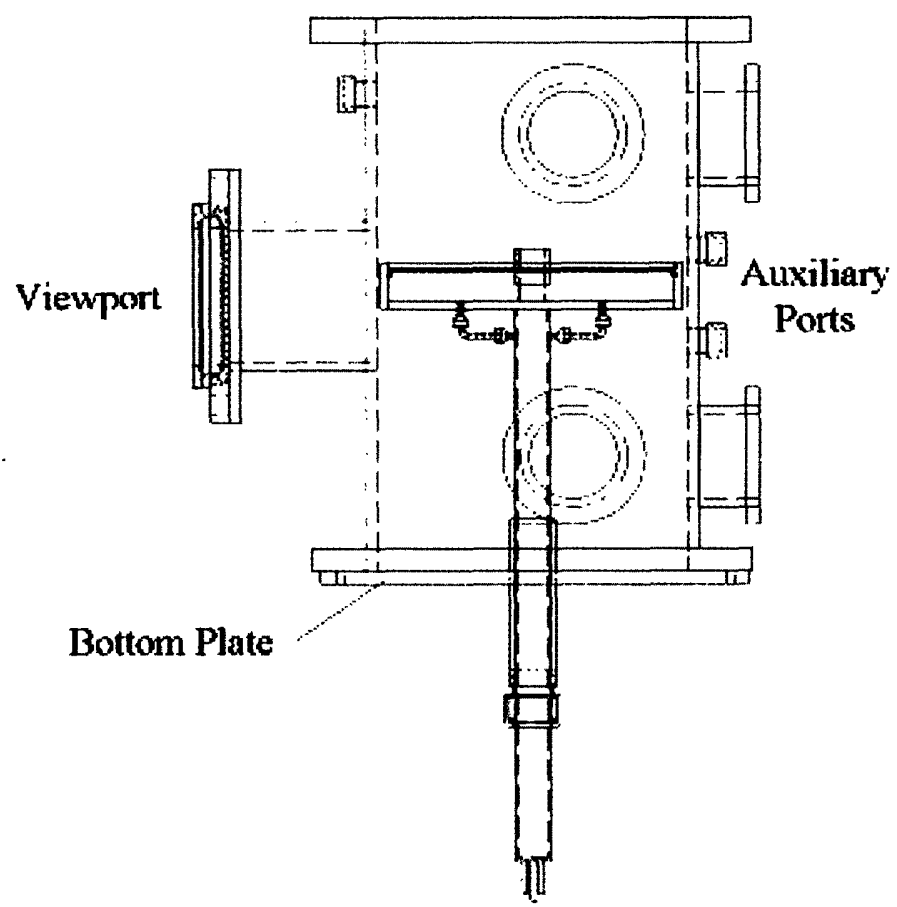

Figure 3-6. Bottom section of diffusion flame chamber with burner and bottom plate.

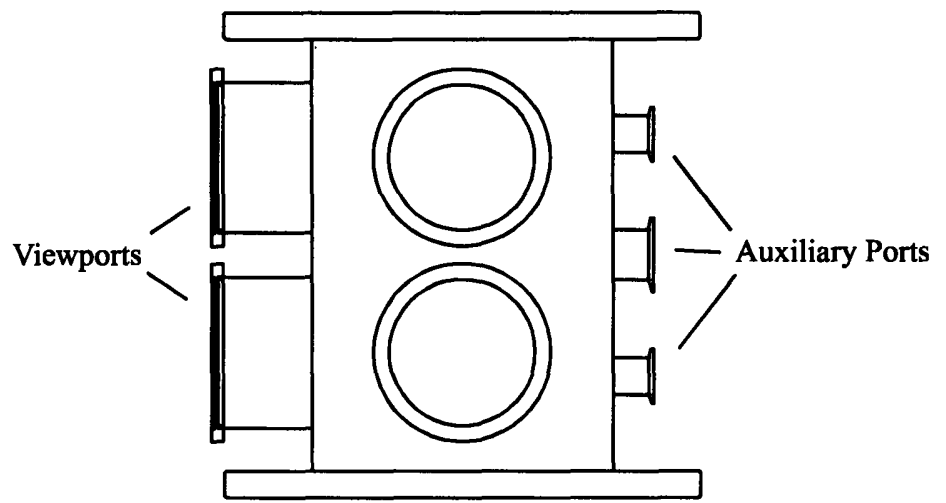

Figure 3-7. One of two identical top sections of diffusion flame chamber.

Like the bottom section, the upper two sections consist of cylindrical stainless steel chambers (11.8 in. ID x 19.4 in.) equipped with two end flanges, three auxiliary ports, and four view ports. The view ports are all equipped with 6-in. glass windows for visual observation. Two of the auxiliary ports are $1 \frac{1}{2} \mathrm{in}$. while the third one is $2 \mathrm{in}$. Again, the auxiliary ports are sealed but provide access points. These sections are 
constructed so they may be used directly between the bottom chamber and top plate without an adapter. One or both of these sections may be removed to accommodate flames of different lengths. For the low-flow flames, only the bottom chamber section was utilized, while all three sections were used for the turbulent flames.

\subsubsection{Top plate}

The top plate, shown schematically in Figure 3-8, consists of a stainless steel disc (18 in. $x \frac{1}{2}$ in) that seals the chamber and contains three $1 \frac{1}{2}$-in.diameter access ports.

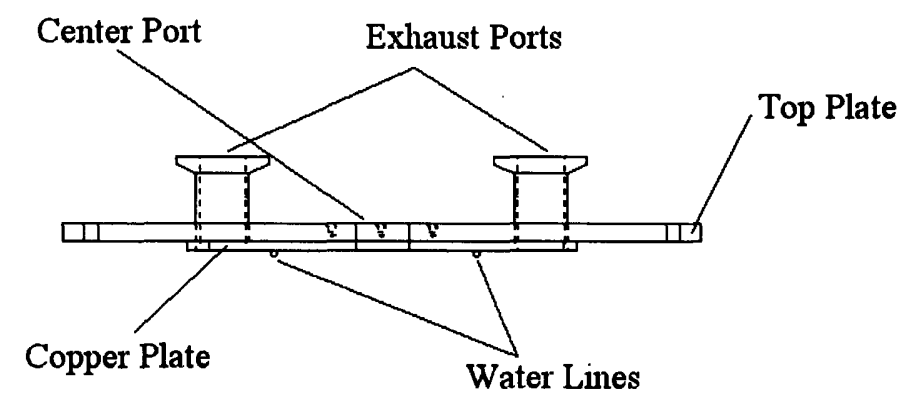

Figure 3-8. Top plate of diffusion flame chamber.

The two outside ports lead to water-cooled exhaust lines feeding to the vacuum pump system and the center port provides access for the flame sampling probe or other accessories. A water-cooled copper disc (11 in. $x$ 1/4 in.) mounted to the bottom of the plate prevents overheating.

\subsubsection{Fuel delivery}

Fuel can be delivered to the burner in either liquid or vapor form. The preferred fuel for fullerene formation are aromatics, which are liquid at room temperature so vaporization is required to feed them as gases. This is not simple for the large flowrates required for turbulent flames and hence, liquid fuel is generally preferred for these. Conversely, when high flowrates are not required, as in laminar flame systems, feeding vaporized fuel is not a problem.

For a vaporized flow, the fuel is initially stored in a 10-liter cylindrical stainless steel reservoir that is heated at the base by a Cimarec (Model 3) 1690-watt hot plate with a magnetic stirrer. The reservoir is fitted with a 2 -in. flange at the top and has an 
adjustable pressure relief valve and a 1/4-in. vapor outlet. An Omega Engineering pressure transducer (Model PX205) connected to the outlet monitors the vapor pressure of the fuel. This transducer is connected to an Omega Engineering Proportional-IntegralDerivative (PID) controller (Model CN76130) that controls the outlet pressure by adjusting the setting of the hot plate. The vapor flow to the chamber is controlled by a heated Tylan mass flow controller (Model VC4900) and can regulate flows up to four liters per minute. Heating of the various vapor lines in the system with heat tape prevents the recondensation of the fuel vapor.

For a liquid flow, the fuel is stored in the 500-mL cylinder of an Isco syringe pump (Model LC5000). The pump is used to deliver a steady and stable flow of liquid fuel to the burner.

In the diffusion flame chamber, the flows of oxygen and argon are controlled by critical orifice meters, while the flow of methane for the ignition flame is controlled by a metering valve. The upstream pressure of each orifice is controlled with a metering valve and monitored with Omega Engineering pressure transducers (Model 242PC100G).

\subsubsection{Quartz sampling probe}

A microprobe sampling system, consisting of a quartz probe with an orifice diameter of about $1.5 \mathrm{~mm}$ and housed in a stainless steel water jacket, is used to collect samples from the flames. The housing of the probe can be seen in Figure 3-1 and the dimensions of the probe are shown in Figure 3-9.

The probe design minimizes the temperature drop from the flame to the probe tip, which minimizes the rate of carbon deposition in the probe orifice and maximizes the flame sampling time. Depending on the sootiness of the flame, this probe design allows for sampling times of up to 5 minutes. The probe is held in a water-cooled jacket and operated at a pressure of approximately 2 Torr, maintained by a Welch (Model 1397) vacuum pump. The flow of noncondensable gas through the probe is collected in a graduated water column where it is measured for use in the calculation of soot and fullerene concentration in the sampled gas. Condensable products, including soot and other compounds are collected on a preweighed filter consisting of glass wool packed into an aluminum foil sleeve. The probe can be inserted into the chamber from either the 
top sampling port or any of the smaller side auxiliary ports of either chamber. This allows the collection of samples from many different locations in the flame. Most often, samples were taken from the side ports in the diffusion chamber.

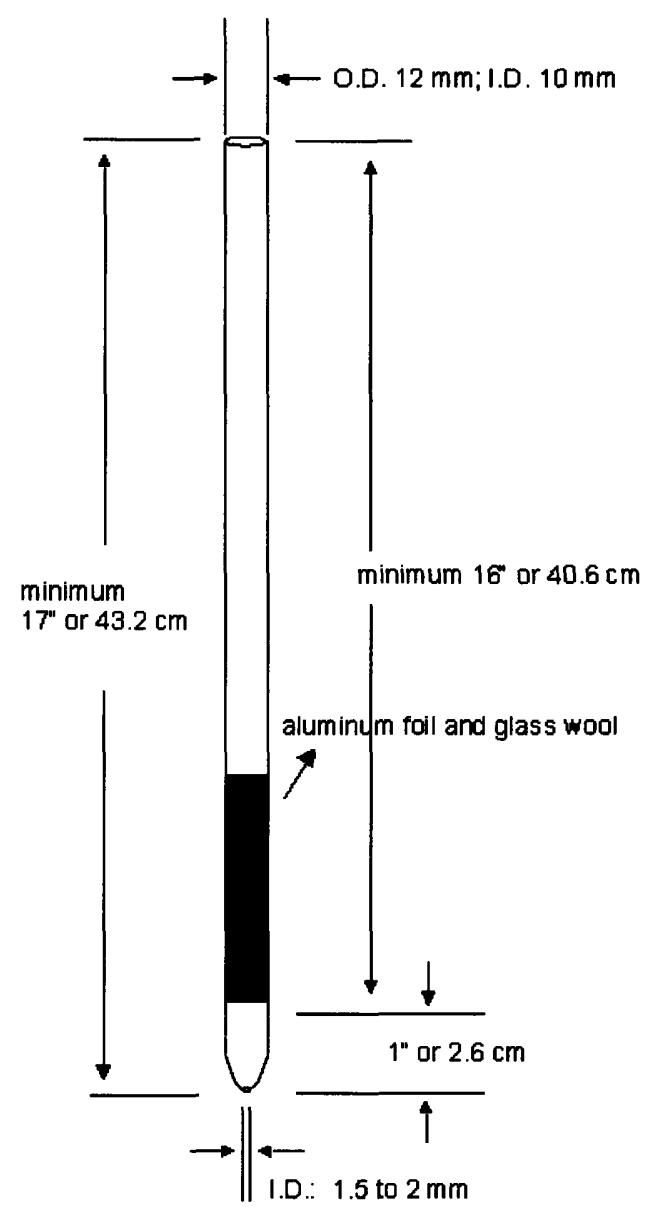

Figure 3-9. Quartz probe design.

\subsubsection{Fullerene extraction}

Once the sampling is completed, the mass of the collected flame material is determined by weighing the filter system again. The contents are then submerged in toluene and ultrasonicated using a Branson Sonifer (Model 450) set at output 2 and 50\% duty cycle for 10 minutes. This ensures that most of the fullerenes present in the condensable material will dissolve into the toluene. Samples of the dispersion are then drawn off for HRTEM analysis and the remainder is vacuum filtered through a $0.45-\mu \mathrm{m}$ 
nylon filter. The resulting solution is then concentrated by nitrogen blowdown to a final volume of one to five milliliters.

\subsubsection{High-performance liquid chromatography}

To analyze the concentration of fullerenes, a 20- $\mu$ l sample of the filtered toluene/fullerene solution is injected into a Hewlett-Packard high-performance liquid chromatography (HPLC) instrument (Model 1050). This instrument is equipped with a quartenary pump and a variable wavelength detector (VWD). A column with 2-(1pyrenyl) ethylsilica stationary phase (Cosmosil Buckyprep, $4.6 \mathrm{~mm} \times 20 \mathrm{~mm}$ ) is used with a toluene flowthrough rate of $1.0 \mathrm{ml} / \mathrm{min}$. Fullerenes and other material can then be identified by the retention times and UV-VIS spectra. Fullerene concentrations are calculated by integrating the area under the peaks and comparing with calibration curves that have been obtained from standard solutions. Nanostructures are too large to be measured by HPLC and are thus analyzed only by HRTEM.

\subsubsection{High-resolution transmission electron microscopy}

A qualitative and quantitative analysis of fullerene and nanostructure formation was performed with HRTEM images. The sonicated sample described in Section 3.1.4 is first subject to further toluene dilution and ultrasonication. A few drops of the ensuing mixture are then placed on an EM grid and the toluene is allowed to evaporate. The grids used are lacey carbon films on a Formvar polymeric substrate on a 200 mesh copper substrate (Ted Pella, Inc.). The HRTEM analyses are carried out on two Jeol microscopes (Models 2000 and 2010) operated at $200 \mathrm{keV}$ with resolutions better than $0.2 \mathrm{~nm}$. In most cases, the images of the material are taken at 590,000 times magnification. Once the images are obtained, the evolution of fullerenic material can be examined. In addition to qualitative analysis, the images are examined quantitatively using an analysis tool developed in MATLAB ${ }^{\mathrm{TM}}$. This tool allows quantification of the radius, length, and curvature distributions of curved layers observed within the fullerenic material.

\subsubsection{Qualitative analysis}

The images obtained from HRTEM analysis are examined for the presence or absence of fullerenic nanostructures and their precursors, and for the scarcity or 
abundance of fullerene-like structures. The relative curvature of observed carbon structures are assessed as well. Although qualitative analysis can be quite subjective, trends and characteristics can be observed rather conclusively. By examining the images in this manner, favorable conditions and locations for fullerenes can be identified and fullerene chemical data obtained by HPLC (Section 3.1.5) can be verified or refuted. To further confirm the qualitative conclusions, though, the images were also examined with a quantitative method (Section 3.1.6.2).

\subsubsection{Quantitative analysis}

The technique used for quantitative analysis of the TEM images, which has been described previously [37, 99], allows for the quantification of radius, length, and curvature of observed carbon structures within the soot material. Figure 3-10 shows a schematic outlining the basics and theory of the technique.
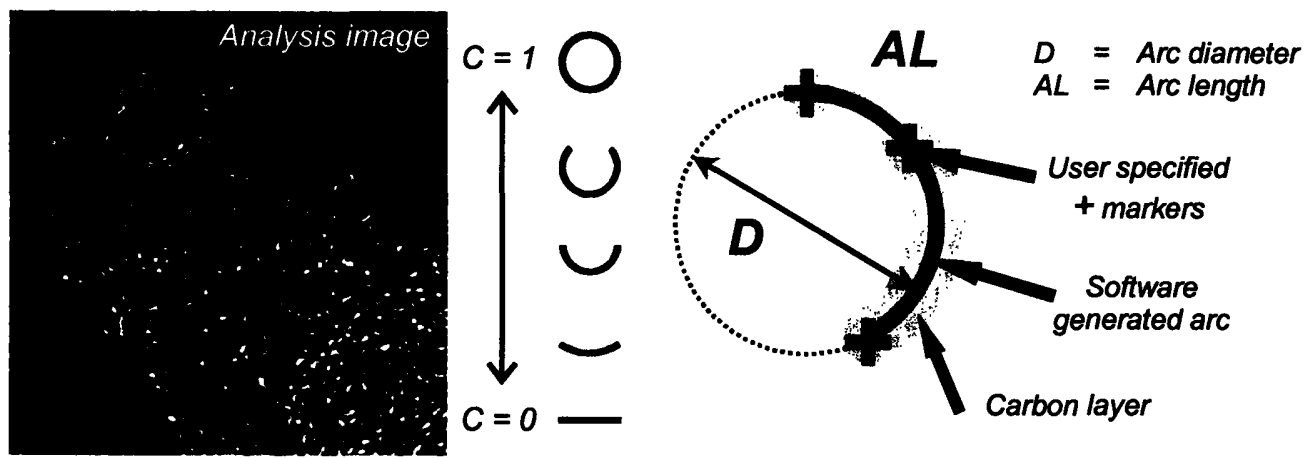

Figure 3-10. Method for quantitative analysis of TEM images.

Using a MATLAB $\otimes$ program that was developed and written by former group members", the user identifies in digitized images three points on a carbon structure, the two end points and any point on the interior. The program measures the length of the specified arc, $L$, the diameter of the apparent circle, $D$, and then calculates the curvature, $C$. The curvature is defined as $C=L / \pi D$ and varies from zero for a flat structure to unity for a closed loop. By examining these three parameters, apparently fullerenic structures can be identified (for $\mathrm{C}_{60}, L=2.1 \mathrm{~nm}, D=0.7 \mathrm{~nm}$, and $C=1$ ). By aggregating the

\footnotetext{
* The program was designed by Dr. Bill Grieco and developed by Dr. David Kronholm and Murray Height, all from the Combustion and Fuels Group at MIT.
} 
measurements of many structures across many images and compiling distributions, trends and characteristics can be observed more objectively than by qualitative analysis alone. However, this technique does not have the capability to measure or account for nanostructures. They unfortunately must still be assessed qualitatively.

\subsection{Low-flow laminar diffusion flames}

The highest yields of fullerenes in flames are obtained under conditions of substantial soot formation. To understand the mechanism of fullerene formation and enable the design of practical combustion systems for large-scale fullerene production, more information on fullerene yields under various conditions is needed. Little work has been reported on the formation of fullerenes in diffusion flames.

To explore the effects of pressure and diluent gas on fullerene yields in diffusion flames, samples of condensable material from laminar diffusion flames under different conditions were collected and analyzed to determine the concentration of fullerenes, to identify nanostructures, and to characterize the curvature of the carbon layers.

\subsubsection{Experimental conditions}

In these flames, a burner cap with an inner diameter of $10 \mathrm{~mm}$ (as described in Section 3.1.1.1) was used as the fuel outlet. The flames consisted of vaporized benzene mixed with an argon diluent burning in an flowing oxygen atmosphere. The cold gas velocity of the unburned benzene/argon mixture, $v_{\mathrm{u}}(\mathrm{fuel} / \mathrm{Ar})$ was 820 or $840 \mathrm{~cm} / \mathrm{s}$, and the argon dilution, $d i l_{\mathrm{Ar}}$, was varied from $65 \%$ to $87.6 \%$. Oxygen was kept at a cold gas velocity, $v_{\mathrm{u}}(\mathrm{oxy})$, of $3.65 \mathrm{~cm} / \mathrm{s}$. The pressure, $p$, in the chamber varied from 12 to 40 Torr. More heavily sooting conditions than those studied here could not be measured due to rapid clogging of the microprobe sample.

To characterize the fullerene production in the flames, the ratios of the total mass of fullerenes to the total mass of condensable material and to the total volume of noncondensable gas were used. In all cases, $\mathrm{C}_{60}$ and $\mathrm{C}_{70}$ accounted for over $95 \%$ of the mass of fullerenes analyzed by chromatograph. Consequently, for data comparison, the sum mass of $\mathrm{C}_{60}$ and $\mathrm{C}_{70}$ was used as representative of the total mass of fullerenes.

Nine different laminar flames were studied here and the parameters of the flames with initial results are shown in Table 3-1. 
In addition to the flame operating conditions, Table 3-1 shows the distance, $h_{\max }$, from the fuel outlet to the probe inlet for the samples with the maximum percentage of fullerenes in the condensable material, the actual maximum percentages, the temperature at the location of these maxima (which was also the temperature maximum for each respective flame), and the flame reference number used in later graphs. The temperatures presented in Table 3-1 are not quite accurate as spectral emissivity has not been considered or accounted for due to an absence of data for these flames. However, it is believed that the data still portray accurate relative differences between the flames and as such, the temperatures were considered only in this manner.

Table 3-1. Laminar diffusion flame parameters

\begin{tabular}{|c|c|c|c|c|c|c|c|}
\hline $\begin{array}{c}P \\
(\text { Torr })\end{array}$ & $\begin{array}{c}v_{\mathrm{u}}(\mathrm{fuel} / \mathrm{Ar}) \\
(\mathrm{cm} / \mathrm{s})\end{array}$ & $\begin{array}{c}d i l_{\mathrm{Ar}} \\
(\% \mathrm{Ar})\end{array}$ & $\begin{array}{c}v_{\mathrm{u}}(\mathrm{oxy}) \\
(\mathrm{cm} / \mathrm{s})\end{array}$ & $\begin{array}{c}h_{\max } \\
(\mathrm{mm})\end{array}$ & $\begin{array}{c}\mathrm{C}_{60}+\mathrm{C}_{70} \\
(\text { mass \%) }\end{array}$ & $\begin{array}{c}T_{\max } \\
(\mathrm{K})\end{array}$ & $\begin{array}{c}\text { Flame } \\
(\text { Ref. \#) }\end{array}$ \\
\hline 20 & 840 & 66.5 & 3.65 & 84.5 & 2.97 & 1659 & 5 \\
\hline 20 & 840 & 71.0 & 3.65 & 74.5 & 6.83 & 1689 & 4 \\
\hline 20 & 840 & 76.0 & 3.65 & 61.5 & 5.13 & 1635 & 3 \\
\hline 40 & 820 & 80.3 & 3.65 & 96.5 & 3.15 & 1694 & 10 \\
\hline 40 & 820 & 82.8 & 3.65 & 88 & 3.73 & 1726 & 8 \\
\hline 40 & 820 & 85.3 & 3.65 & 74 & 4.49 & 1754 & 11 \\
\hline 40 & 820 & 97.6 & 3.65 & 69 & 4.86 & 1694 & 12 \\
\hline 12 & 820 & 65.0 & 3.65 & 57 & 12.44 & 1599 & 13 \\
\hline 12 & 820 & 67.5 & 3.65 & 53 & 12.40 & 1583 & 14 \\
\hline
\end{tabular}

\subsubsection{Fullerene and soot results}

The highest soot concentration in diffusion flames is in the wings of a flame, where the radial fuel and oxygen concentration gradients are highest and the temperature is maximum. Further from the flame axis, the soot is consumed by oxidation. The flame wings collapse to one point at the top of the stoichiometric surface, where the flame reaches its highest overall temperature; therefore, the axial concentration gradients of fuel, oxygen, and products including soot are similar to the radial gradients closer to the 
burner. Aggregate results from these flames indicate that fullerene concentration sees a peak with respect to argon dilution but increases at lower pressures and shorter flames. Consistent with analytical results, soot structure evolution results show that the soot becomes more ordered and more curved with increasing residence time (analogous to height above burner) until the fullerene maximum, at which point, curvature decreases again. Nanostructures were also found at long residence times after the fullerene peak.

\subsubsection{Fullerenes}

Results from the three 20 Torr flames with different argon dilutions are shown in Figure 3-11(a) and (b). The amount of $\mathrm{C}_{60}$ and $\mathrm{C}_{70}$ as a mass percentage of the condensables is shown in Figure 3-11(a), and the concentration of $\mathrm{C}_{60}$ and $\mathrm{C}_{70}$ in the cold sampled gas is shown in Figure 3-11(b), both versus the height above the burner, $h$.

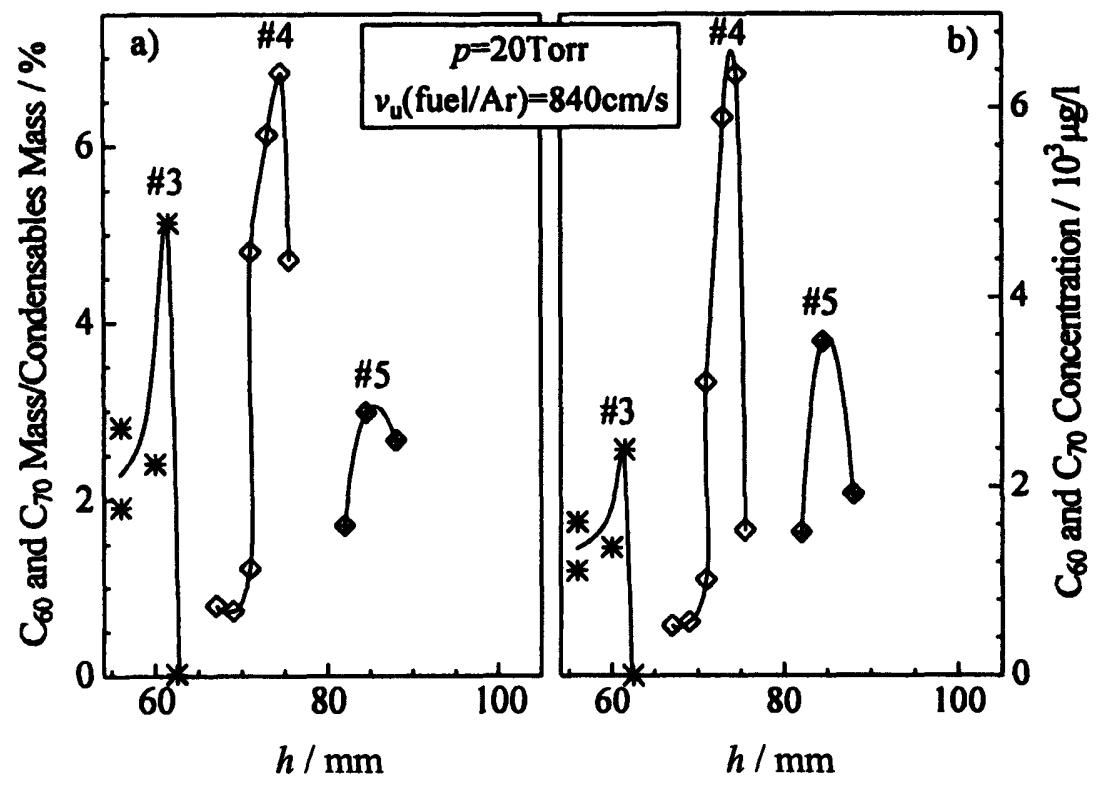

Figure 3-11. Quantity of fullerenes $C_{60}$ and $C_{70}$ in the condensable material (a) and concentration of fullerenes $C_{60}$ and $C_{70}$ in the cold probed gas (b) from different heights above the burner in different laminar diffusion flames at $\mathrm{p}=20$ Torr.

The highest concentration concentration of fullerenes always occurred just above the visible stoichiometeric surface of the flame, where the temperature maximum also occurs. Therefore, the locations of the maxima of the curves are indicative of the stoichiometric flame length. Figure 3-11(a) and (b) and Table 3-1 show that diluting the benzene with argon results in a shorter flame but not necessarily in a lower temperature, 
and that a higher fullerene percentage does not necessarily indicate a higher fullerene concentration. A decrease in the concentration of condensable material can be large enough to offset the increase in fullerene percentage.

Estimation of the percentage of the carbon feed that is converted into fullerenes from the flame \#4 data gives $0.5 \%$. This value, for non-optimized conditions, is comparable to the largest value observed in premixed benzene flames $[85,86]$.

Data for flames at 40 Torr (Figure 3-12(a) and (b)) compared with those for 20 Torr (Figure 3-11(a) and (b)) show that increasing the pressure increases the flame length while decreasing the flame diameter, resulting in a more heavily sooting flame. In general, the percentage of fullerenes is lower at 40 Torr than at 20 Torr. At lower dilutions than those shown in Figure 3-12(a) and (b), large soot concentrations at 40 Torr caused rapid probe clogging. With increasing dilution, the 40 Torr flames show an increase in the maximum percentage of fullerenes (Figure 3-12(a)) and a decrease in the maximum fullerene concentration (Figure 3-12(b)). Although measurements were taken at dilutions up to $87.6 \%$, where the temperature is lower than that of the next lower dilution, the peak in percentage of fullerenes is still higher (discussed in Section 3.2.3.1). At lower dilutions, the concentration would be expected to decrease with decreasing dilution because the flame temperature goes down due to increased soot radiation. Such behavior is seen premixed flames $[85,86]$, where the concentration of fullerenes decreases with decreasing fuel dilution over a specified range of dilutions.

Two flames were studied at 12 Torr (Table 3-1). Consistent with the other results, the flames were shorter and the percentage of the fullerenes was higher than at 20 Torr.

The maximum percentage of fullerenes in the condensable material from the different flames decreases with increasing flame length in a strong relation, as shown in Figure 3-13(a). Shorter flames, which result from higher dilution or lower pressure, favor fullerenes over soot, as has also been suggested based on behavior seen in premixed flames [89]. This consumption should be slower for lower pressure and higher dilutions because of lower soot concentrations. The effect of a lower precursor concentration seems to be overwhelmed (discussed in Section 3.2.3.1). 


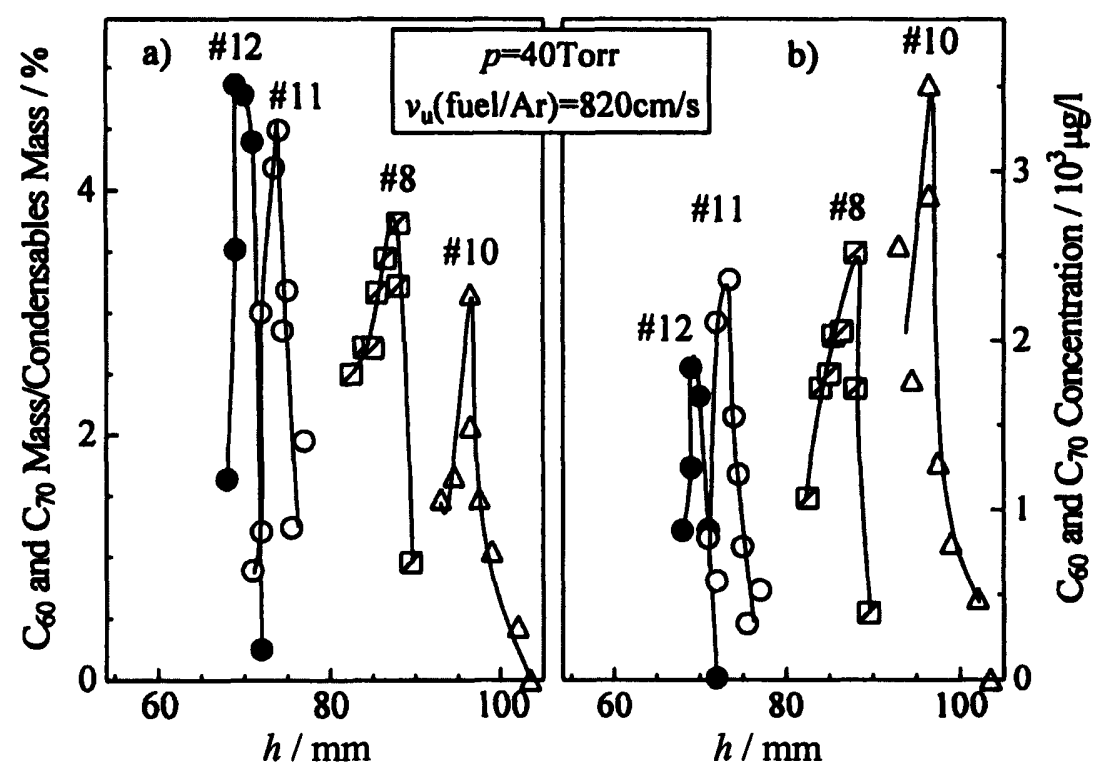

Figure 3-12. Quantity of fullerenes $C_{60}$ and $C_{70}$ in the condensable material (a) and concentration of fullerenes $C_{60}$ and $C_{70}$ in the cold probed gas (b) from different heights above the burner in different laminar diffusion flames at $\mathrm{p}=40$ Torr.
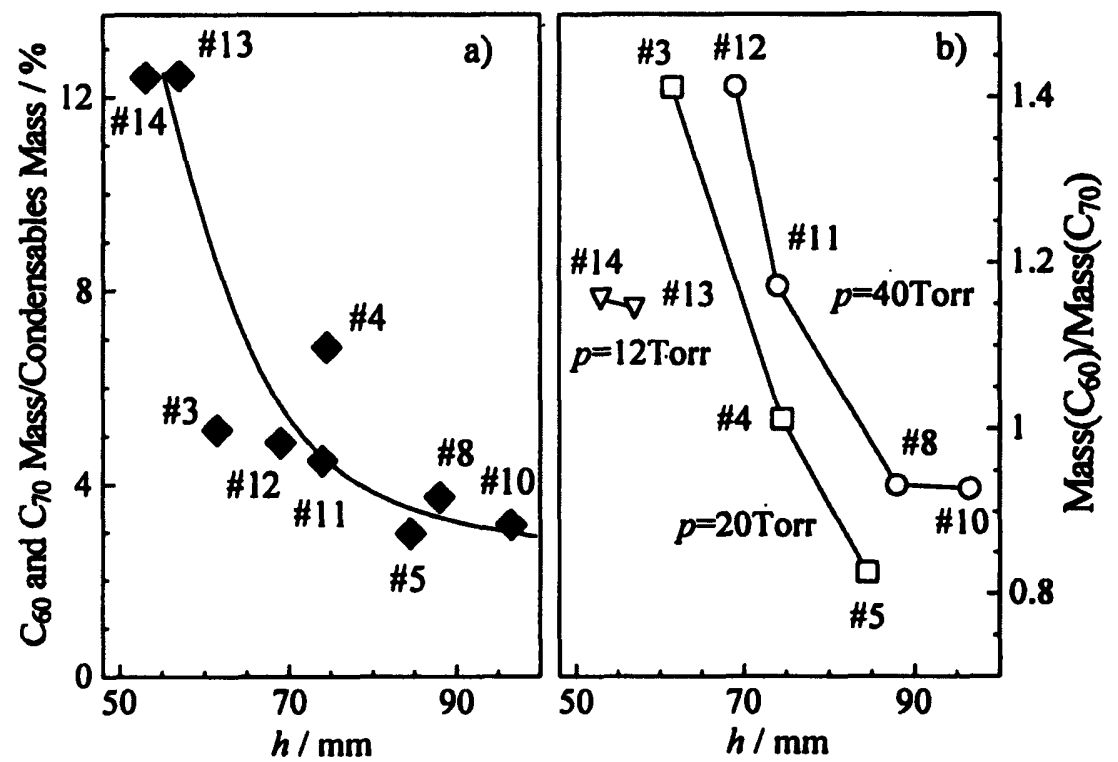

Figure 3-13. Maximum amount of fullerenes $C_{60}$ and $C_{70}(a)$ and mass ratio of $C_{60}$ to $C_{70}$

(b) in the condensable material at the height above the burner corresponding to maximum fullerene concentration in laminar diffusion flames, at different pressures ( $p$ ) and different argon dilutions $\left(\mathrm{dil}_{A r}\right)$.

For each set of flames at different pressures, the mass ratio of $\mathrm{C}_{60}$ to $\mathrm{C}_{70}$ at the maximum fullerene concentration for each flame increases with increasing dilution 
(Figure 3-13(b)). However, unlike the fullerene percentage trend, this effect is pressure dependent.

\subsubsection{Soot structure evolution}

An example of an HRTEM micrograph of soot from flame \#11 (75 mm) is shown in Figure 3-14 (the white color in the images represents carbon layers or shells). The inset shows an enlarged area with closed shells. Significantly more ordered and more curved carbon layers of shells were seen in this study than in premixed benzene combustion [99].

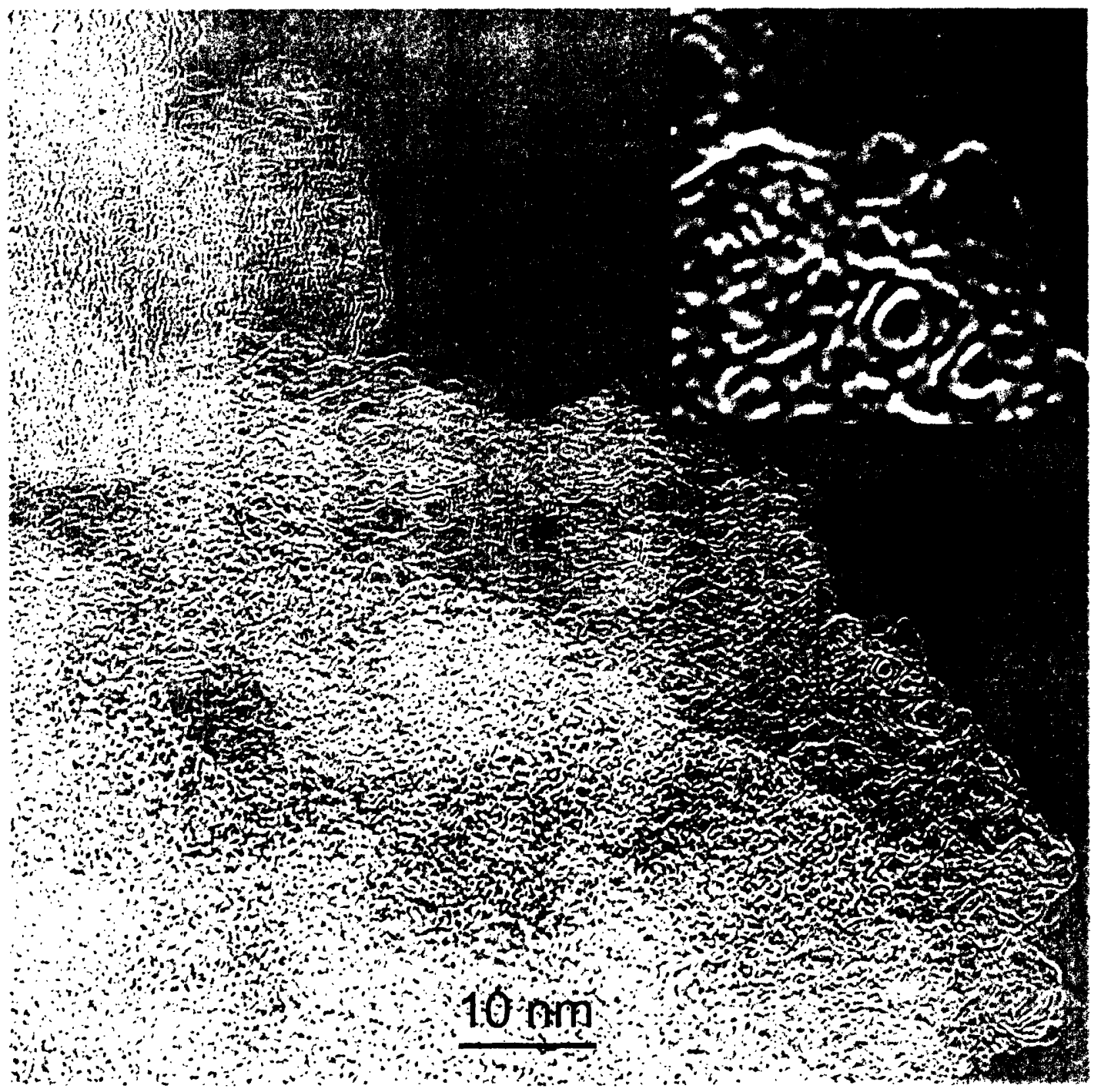

Figure 3-14. Representative HRTEM image of material collected at $\mathrm{h}=75 \mathrm{~mm}$ in flame $\# 11$ ( $\mathrm{p}=40$ Torr, $\mathrm{v}_{u}\left(\right.$ fuel/Ar) $=820 \mathrm{~cm} / \mathrm{s}, \mathrm{v}_{u}($ oxy $\left.)=3.65 \mathrm{~cm} / \mathrm{s}, \operatorname{dil}_{A r}=85.3 \%\right)$. 
Figure 3-15 shows soot from four different heights above the burner that were used from curvature analysis. Carbon structures along the periphery of the soot particles were analyzed quantitatively to determine the evolution of the structures with increasing height above the burner. Only the periphery of the particles was thin enough to allow for accurate measurements of the layers since the interior of the particle contained many stacked layers of carbon material. The hand-drawn lines show the boundary between the outside areas that are counted and the particle interior.

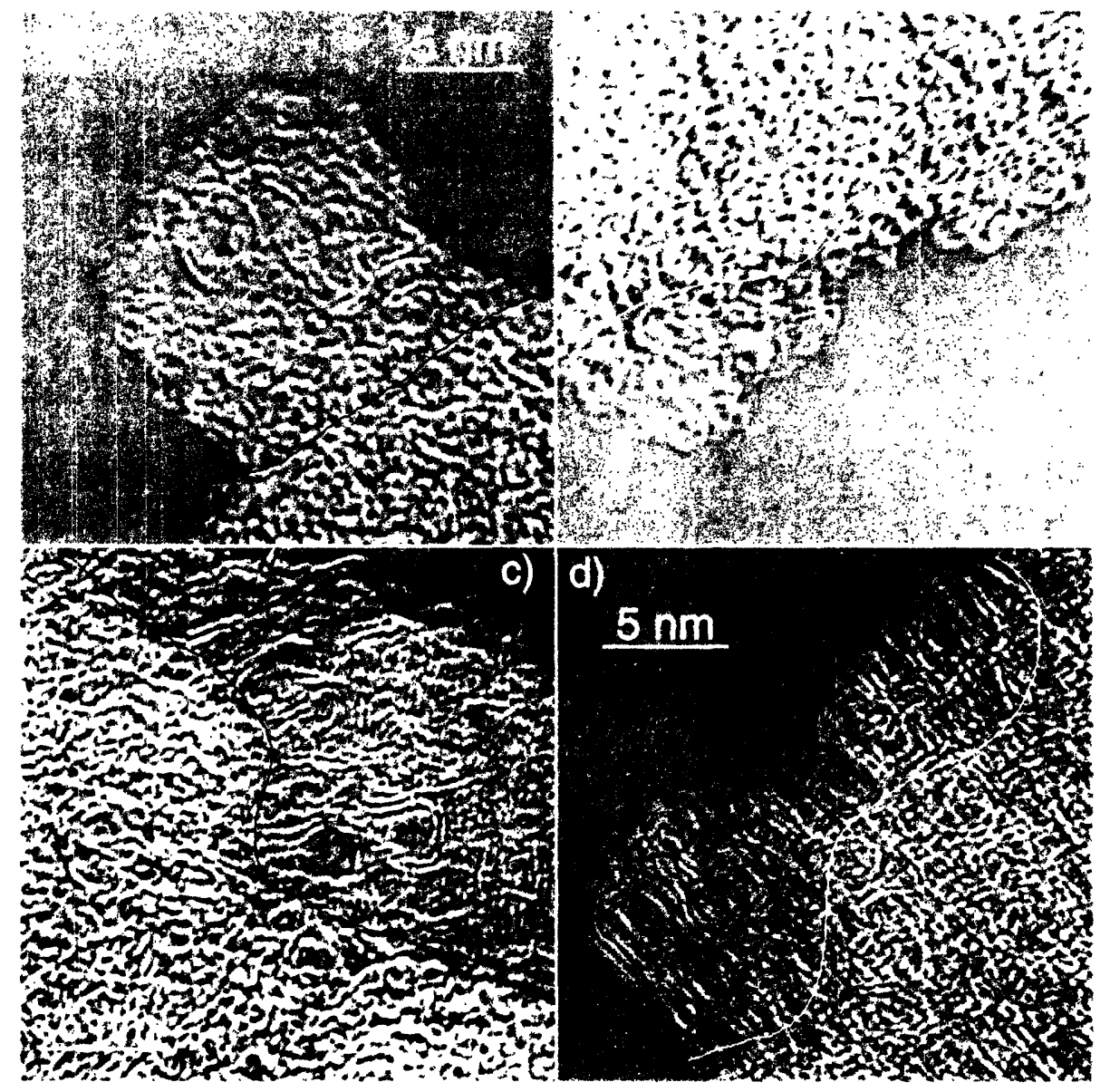

Figure 3-15. Representative counted material from soot collected at $\mathrm{h}=72 \mathrm{~mm}$ (a), $74 \mathrm{~mm}$ (b), $75 \mathrm{~mm}$ (c), and $77 \mathrm{~mm}$ (d) in flame \#11 (p=40 Torr, $\mathrm{v}_{u}(\mathrm{fuel} / \mathrm{Ar})=820 \mathrm{~cm} / \mathrm{s}$,

$$
\mathrm{v}_{u}(\text { oxy })=3.65 \mathrm{~cm} / \mathrm{s}, \operatorname{dil}_{A r}=85.3 \% \text { ). }
$$

Two properties of the curved layers or shells, arc length $(L)$ and diameter $(D)$, were measured directly and used to calculate a non-dimensional parameter, curvature $(C=L / \pi D)$. Normalized histograms of each of these parameters and scatter plots of arc length versus diameter for each of the four samples and amorphous carbon (for 
comparison) are shown in Figures 3-16 thorough 3-20. The truncation of the $x$-axes in these figures causes some of the histograms to sum to less than 100 (nothing of importance was excluded).
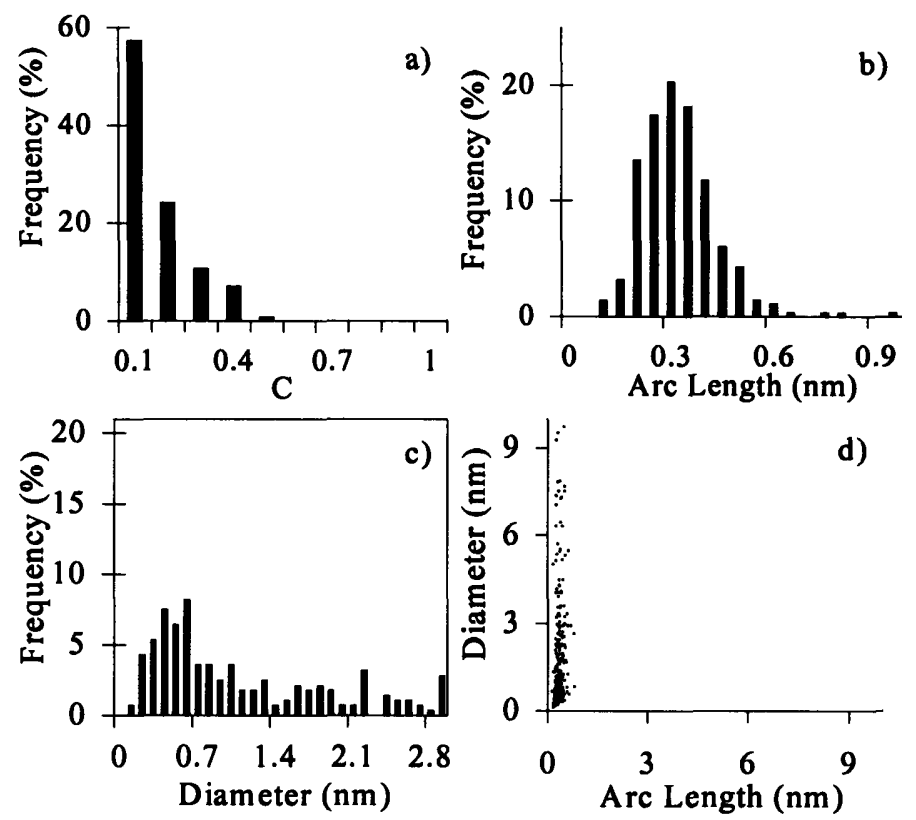

Figure 3-16. Normalized histograms of curvature (a), arc length (b), and diameter (c) and arc length versus diameter scatter plot (d) of amorphous carbon material.
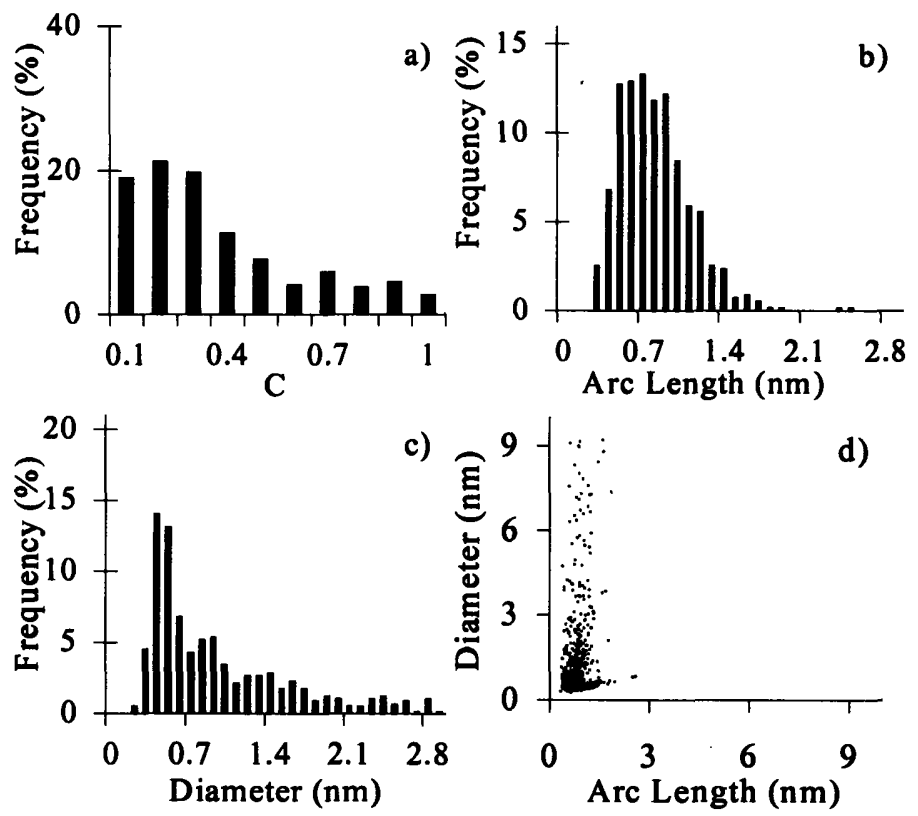

Figure 3-17. Normalized histograms of curvature (a), arc length (b), and diameter (c) and arc length versus diameter scatter plot (d) of condensable material collected at $\mathrm{h}=72$ $\mathrm{mm}$ in flame $\# 11$ ( $\mathrm{p}=40$ Torr, $\mathrm{v}_{u}(f u e l / A r)=820 \mathrm{~cm} / \mathrm{s}, \mathrm{v}_{u}($ oxy $\left.)=3.65 \mathrm{~cm} / \mathrm{s}, \operatorname{dil}_{A r}=85.3 \%\right)$. 

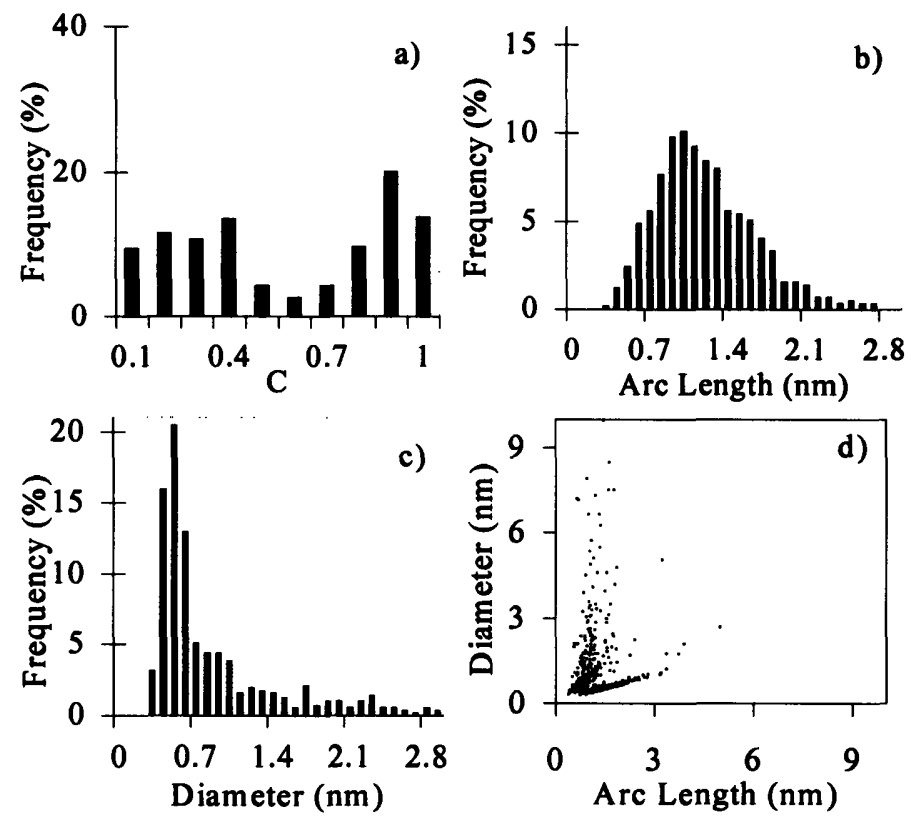

Figure 3-18. Normalized histograms of curvature (a), arc length (b), and diameter (c) and arc length versus diameter scatter plot (d) of condensable material collected at $\mathrm{h}=74$ $\mathrm{mm}$ in flame \#11 (p=40 Torr, $\mathrm{v}_{u}($ fuel $/ A r)=820 \mathrm{~cm} / \mathrm{s}, \mathrm{v}_{u}($ oxy $\left.)=3.65 \mathrm{~cm} / \mathrm{s}, \operatorname{dil}_{A r}=85.3 \%\right)$.
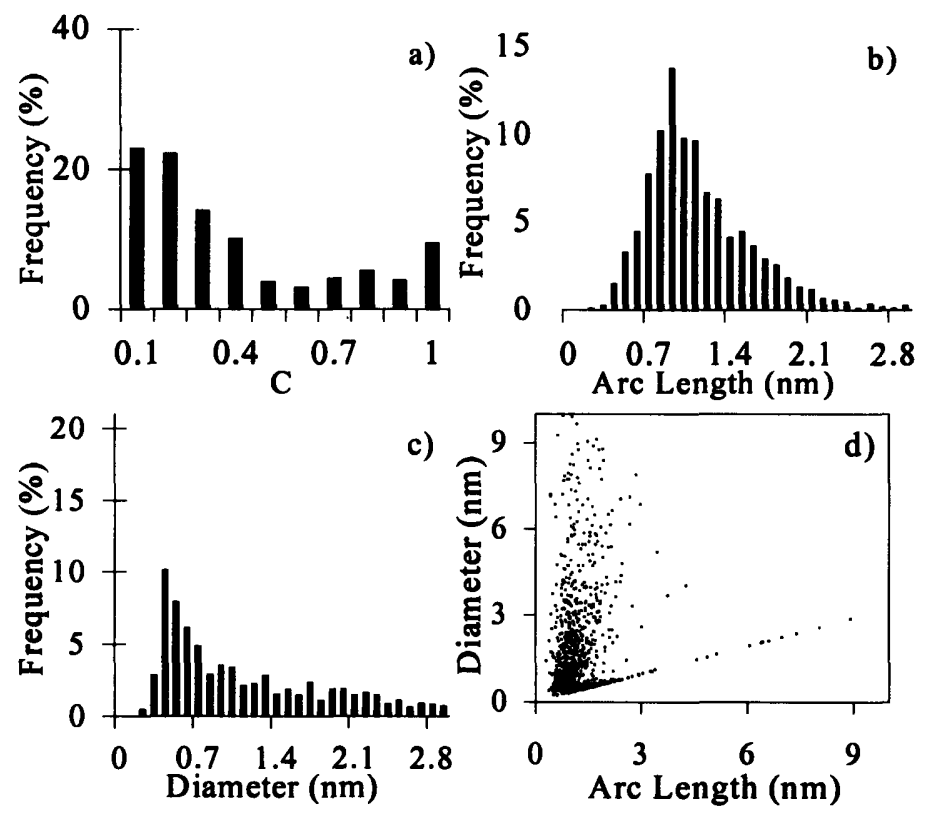

Figure 3-19. Normalized histograms of curvature (a), arc length (b), and diameter (c) and arc length versus diameter scatter plot $(d)$ of condensable material collected at $\mathrm{h}=75$ $\mathrm{mm}$ in flame \#11 (p=40 Torr, $\mathrm{v}_{u}($ fuel $/ \mathrm{Ar})=820 \mathrm{~cm} / \mathrm{s}, \mathrm{v}_{u}($ oxy $\left.)=3.65 \mathrm{~cm} / \mathrm{s}, \mathrm{dil}_{A r}=85.3 \%\right)$. 

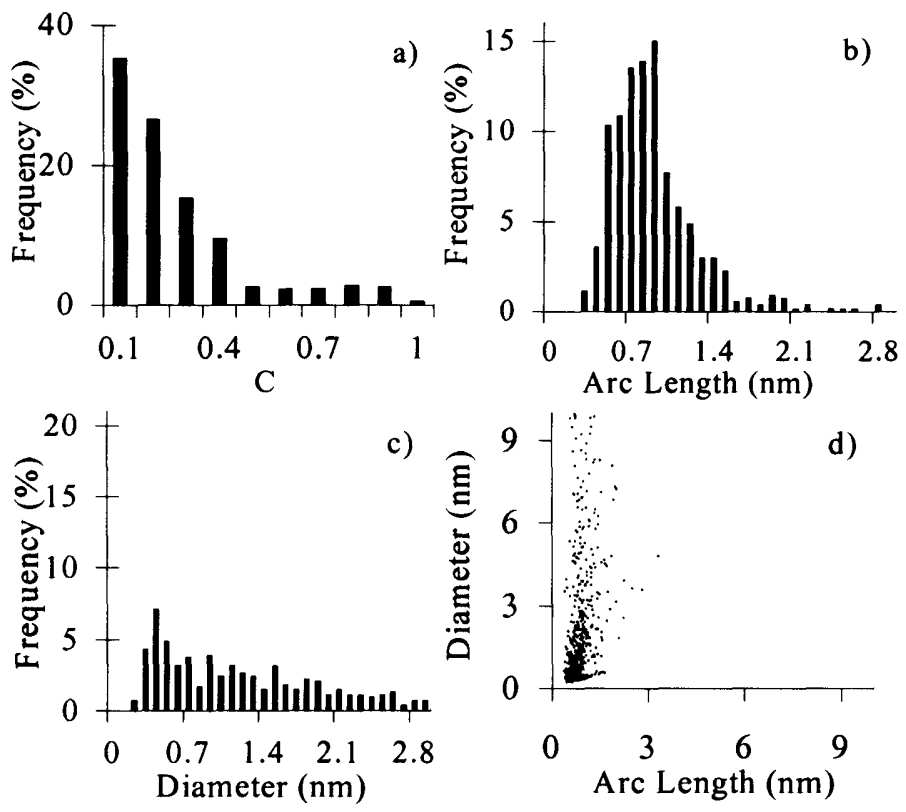

Figure 3-20. Normalized histograms of curvature (a), arc length (b), and diameter (c) and arc length versus diameter scatter plot (d) of condensable material collected at $\mathrm{h}=77$ $m m$ in flame \#11 ( $\mathrm{p}=40$ Torr, $\mathrm{v}_{u}($ fuel $/ A r)=820 \mathrm{~cm} / \mathrm{s}, \mathrm{v}_{u}($ oxy $\left.)=3.65 \mathrm{~cm} / \mathrm{s}, \mathrm{dil}_{A r}=85.3 \%\right)$.

It can be seen that the structures in the amorphous carbon sample, compared with those of any flame sample, have very low curvatures and relatively short arc lengths. The large diameters seen in the amorphous carbon are due to the relatively flat nature of the structures. As a consequence of the analysis method, the flatter of two structures with equal arc lengths will have a greater measured diameter.

In Figure 3-21, a comparison of the curvature histograms in parts a in Figures 316 through 3-20 shows that curvature increases with distance until the fullerene maximum $(74 \mathrm{~mm})$, where it then decreases. This indicates that the observed fullerene peak is also the peak for the generation of completely closed shell structures. The histograms in parts $b$ of the figures show a similar behavior in that arc lengths increase until a dropoff is observed after the fullerene maximum. The part $\mathrm{c}$ histograms show a decrease in diameter with increasing distance until the fullerene maximum, followed by an increase. The diameter peak is seen at $0.7 \mathrm{~nm}$, the diameter of a $\mathrm{C}_{60}$ molecule, and is highest at $74 \mathrm{~mm}$, the becoming less pronounced after the fullerene maximum. In the scatter plots of parts $d$, points that lie on the delineated lower boundary represent completely closed-shell structures. It appears that larger closed-shell structures are more prevalent with increasing distance until they practically disappear at $77 \mathrm{~mm}$. 
Considering these trends collectively, it can be seen that the carbon structures in the soot become more curved and more closed until the fullerene peak, then change to flatter, smaller structures.

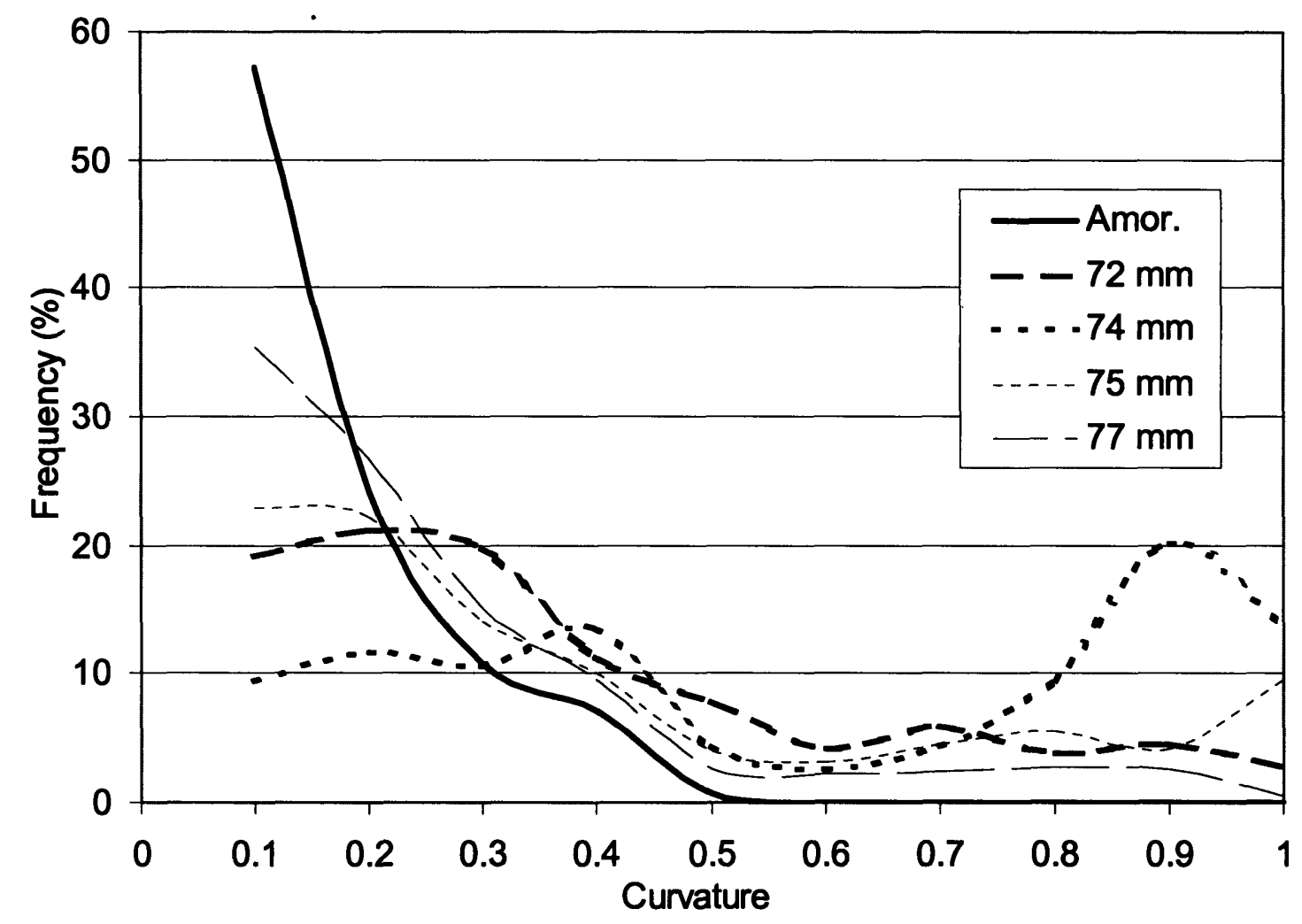

Figure 3-21. Curvature comparison for flame \#11 (p=40 Torr, $\mathrm{v}_{u}($ fuel/Ar) $=820 \mathrm{~cm} / \mathrm{s}$, $\mathrm{v}_{u}($ oxy $\left.)=3.65 \mathrm{~cm} / \mathrm{s}, \operatorname{dil}_{A r}=85.3 \%\right)$.

The sample images were examined qualitatively for the emergence of fullerenic nanostructures. Figure 3-22 shows a micrograph representative of the nanostructures from flame \#13 at $61 \mathrm{~mm}$. The areas that resemble amorphous carbon in Figure 3-22 are the lacey carbon grid supports on which the samples are deposited. The structures consist of stacked layers of fullerenic carbon and include nanotubes and highly ordered onionlike structures. The inset in Figure 3-22 shows an enlarged onion-like closed-shell nanostructure. Similar structures have been seen in fullerene-forming premixed benzene flames [99, 112-114]. 


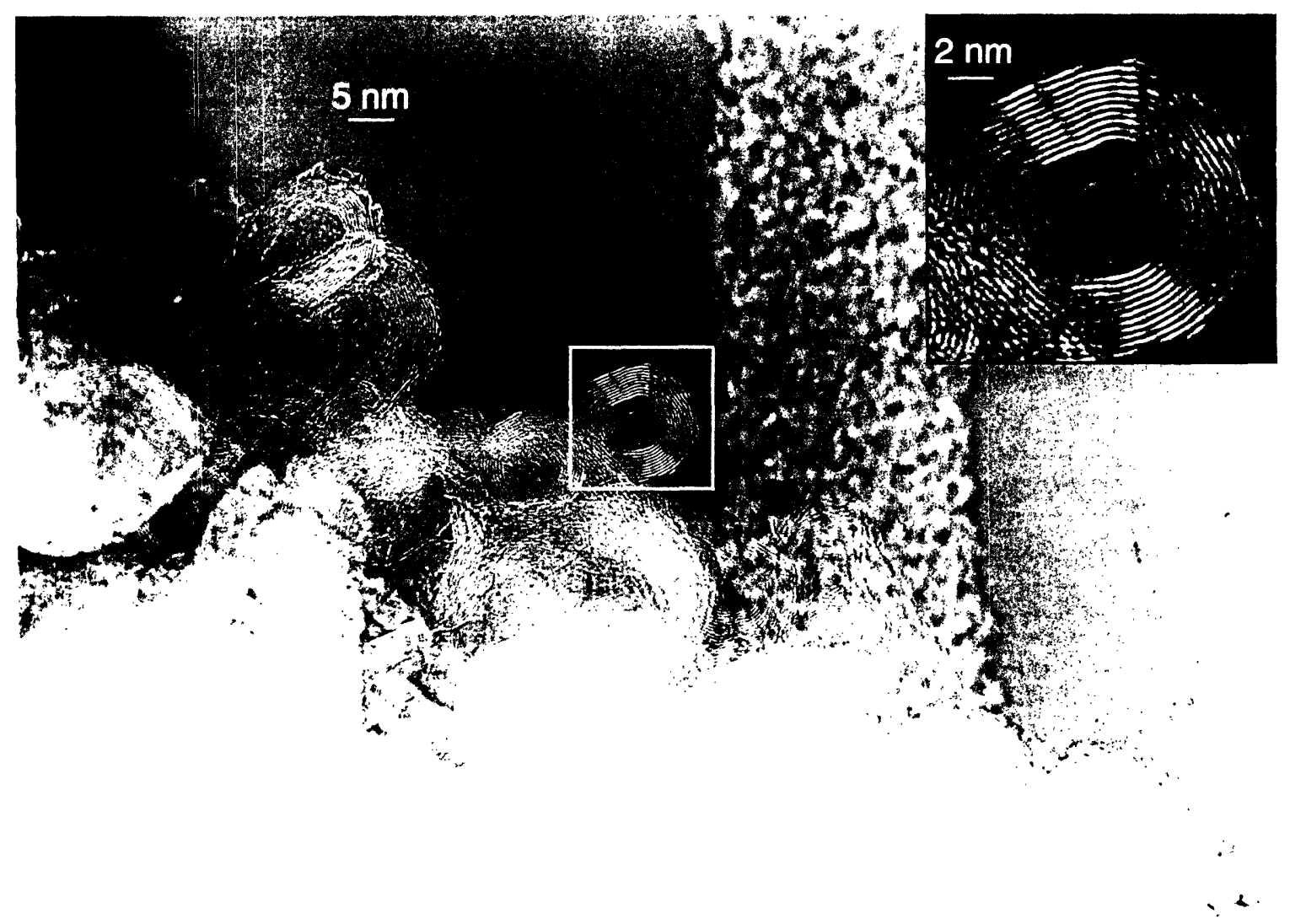

Figure 3-22. HRTEM image of fullerenic nanostructures in condensable material collected at $\mathrm{h}=61 \mathrm{~mm}$ in flame $\# 13\left(\mathrm{p}=12\right.$ Torr, $\mathrm{v}_{u}($ fuel $/ \mathrm{Ar})=820 \mathrm{~cm} / \mathrm{s}, \mathrm{v}_{u}($ oxy $)=3.65$ $\mathrm{cm} / \mathrm{s}, \operatorname{dil}_{A r}=65.0 \%$ ).

\subsubsection{Discussion}

The results from the samples taken from the laminar diffusion flames indicate that high levels of dilution are needed to achieve optimal concentrations of fullerenes and that fullerene production is sensitive to both pressure and dilution changes and is subject to competing effects. In addition, data obtained from the soot micrographs are consistent with the chemical analyses and indicate that fullerenes are being incorporated into the soot and into nanostructures. Quantitative determinations, however, for maximum fullerene production and nanostructure formation are unfortunately still not possible.

\subsubsection{Fullerenes}

The elimination of $\mathrm{CO}$ from oxidized $\mathrm{PAH}$ is thought to be a source of fivemembered rings [115] in the structure of combustion-generated $\mathrm{PAH}$, which are precursors to fullerenes in flames $[32,92,116]$. However, the highest concentration of 
fullerenes is in the region of the flame where the precursor concentration is decreasing due to oxidation. Oxidation reactions are also responsible for the decomposition of the fullerenes at greater distances.

That the maximum fullerene percentage is at such high fuel dilutions as those seen here may indicate the fullerene consumption by soot is important. At 40 Torr (Figure 3-12(a) and (b)), the percentage of fullerenes is highest at the highest dilution, yet the temperature is lower compared with lower dilutions. Both the overall carbon concentration in the flame and the soot formation decrease with increasing dilution. The lower soot concentrations at higher dilutions reduce radiative heat loss from the flame, partially offsetting the lower heat production due to less fuel. At the same time, the concentration of precursors for fullerenes, as well as soot, is decreasing, but the flames with the lowest total concentration exhibit the highest fullerene percentage. Similarly, decreasing pressure lowers total soot and fullerene concentration but still raises the percentage of fullerenes. Associated with these effects is the observed strong correlation in which shorter flames yield higher percentages of fullerenes in the condensable material.

Although this provides a qualitative explanation for the appearance of a maximum rate of fullerene formation, a quantitative prediction for the optimal dilution is not yet possible. It is possible, however, to establish empirical guidelines based on the combustion parameters in this work.

\subsubsection{Soot structure evolution}

The electron microscope images show a soot microstructure that is different from samples collected from higher pressure flames as well as low-pressure premixed flames [99]. Structures similar to those seen here have also been found in commercially produced carbon black $[100,101]$. Quantitative analysis of the micrographs using the curvature parameter indicates that carbon layers or shells in the soot become more curved (i.e., more fullerenic) with increasing distance in the flame up to the fullerene maximum, after which the curvature declines (Figures 3-16 through 3-21). The arc length increases with distance, indicating growth to larger structures, while diameter decreases, indicating an increase in curvature of layers already present in the soot or the addition to the soot of 
smaller-diameter fullerenic carbon. Together, these trends indicate that gas-phase-formed fullerenes are consumed by, and presumably incorporated into, the soot and nanostructures. This behavior is consistent with the hypothesis that fullerenes added to the soot from the gas-phase are a source of the fullerenic structures, including closed shells, of around $0.7 \mathrm{~nm}$ diameter seen in the soot micrographs. The added fullerenes might also contribute, along with other PAH in the soot, to the growth of larger curved layers indicated by the increase of arc length. The correspondence of the diminishing of the $0.7 \mathrm{~nm}$ diameter peak and the fullerene maximum is also consistent with the fullerene-addition hypothesis.

The quantity of nanotubes and onion-like structures (Figure 3-22) seen here is higher than that observed in premixed flames [99], where nanostructures are seen only at long residence times after oxygen depletion. In our flames, nanostructures appear soon after the stoichiometric flame surface where the maximum temperature occurs and the fullerenes and soot are being oxidized. Consequently, the timescale for nanostructure formation in laminar diffusion flames must be shorter than that for premixed combustion. It can be concluded that fullerenic nanostructures are formed after the fullerene peak where soot and fullerenes are being consumed by oxidation. The images also appear to show nanostructure precursors that were being formed from the soot material.

\subsubsection{Summary}

The evolution of fullerenes, fullerenic soot material, and fullerenic nanostructures with increasing distance from the fuel outlet, as concluded by the findings in this work, can be summarized as follows:

Prior to the stoichiometric surface:

- Pre-C60 incubation

- Presence of relatively flat soot structures

- Concentration of $\mathrm{C}_{60} / \mathrm{C}_{70}$ increasing with distance

- Curvature of soot structures increasing with distance

Just above the stoichiometric surface:

- Peak of $\mathrm{C}_{60} / \mathrm{C}_{70}$ concentration

- Maximum temperature 
- Maximum soot structure curvature

- Presence of nanostructure precursor cells

After stoichiometric surface:

- Fullerenes consumed by oxidation

- Fullerenes incorporated into nanostructures

- Curvature of soot structures decreasing with distance

- Concentration of $\mathrm{C}_{60} / \mathrm{C}_{70}$ decreasing with distance

- Appearance of fullerenic nanostrucutres

\subsection{High-flow turbulent-like diffusion flames}

The work on laminar diffusion flames indicates that higher temperatures lead to increased fullerene formation. Since turbulent flames in general burn hotter than laminar flames fullerene formation was studied in diffusion flames under turbulent-like conditions. The term 'turbulent-like' is used here because the calculated Reynolds number indicated that the flame is still in the laminar regime. However, visual observations of the flame reveals flow eddies and erratic behavior making the flames appear turbulent. For brevity, 'turbulent' will be used to refer to the flames described in this section.

Other advantages to using a turbulent flame include higher heat transfer rates, greater mixing leading to higher temperatures, as well as a greater mass of fuel per unit volume of flame, both of which contribute to a higher fullerene-producing potential. In addition, although they have not been performed before, turbulent flame studies are important, as any commercial fullerene-producing combustion process would most likely use turbulent diffusion flames.

It was believed that turbulent diffusion flames would be promising for fullerene formation but experimental results have proven otherwise. While fullerene production is possible in a large flame region, the yields are minimal with no observable maxima or trends with increasing residence times. It was also seen that varying oxygen and argon flowrates had a limited effect on the turbulent flame fullerene yield. 


\subsubsection{Experimental conditions}

Initial experiments involved the use of a Hago oil burning spray nozzle (Model $.30-45^{\circ}-\mathrm{B}$ ) inside the burner at the top of the fuel/diluent feed line. This nozzle produced a $45^{\circ}$ spray of fuel at the nozzle tip. Liquid benzene fuel was injected using a syringe pump to reach the desired flowrates. Pure fuel was fed to the system since the argon diluent is vapor and a two-phase feed was unfeasible with this nozzle. Initially, the chamber was operated at a pressure of 20 Torr, a fuel flowrate of $408 \mathrm{ml} / \mathrm{h}$, and an oxygen flowrate of 19 SLPM. These conditions give a Reynolds number of only about 700 , indicating that flow is not yet turbulent (the Reynolds number here is computed using the observed flame diameter and the velocity of all gases being injected into the flame). However, these initial experiments indicated that turbulent flames are promising for fullerenes.

Based on these results, turbulent flames using the Spraying Systems Co. nozzle discussed in Section 3.1.1.1 were studied. Again, the liquid benzene flowrate was set to $408 \mathrm{ml} / \mathrm{h}$ and the chamber pressure was set to 20 Torr. The argon dilution varied from $70.1 \%$ to $77.5 \%$ and the equivalence ratio, which was controlled by adjusting the oxygen flowrate, from 0.889 to 1.27 . More heavily sooting conditions could not be studied due to flame instability. These conditions also give Reynolds numbers of around 700-800 but visually, the flames appeared to be turbulent. For these flames, both chamber extensions were used and the sampling probe was positioned in the center of the flame and introduced from the side. The height above burner was varied by vertically adjusting the burner. Like the laminar study, the collected samples were analyzed by HPLC and by HRTEM.

Four different turbulent flames were studied and the operating parameters of the flames are shown in Table 3-2. The four flames shown in Table 3-2 cover each combination of low vs. high oxygen flowrate and low vs. high dilution percentage. The methane flow was increased or decreased in conjunction with the argon to ensure stability of the flame system. The quoted equivalence ratios include the carbon added by the methane fuel. The reference numbers are used for flame identification in the following sections. 
Table 3-2. Turbulent diffusion flame parameters

\begin{tabular}{|c|c|c|c|c|c|c|c|c|}
\hline $\begin{array}{c}\text { Pressure } \\
(\text { Torr })\end{array}$ & $\begin{array}{c}\text { Benzene } \\
(\mathrm{ml} / \mathrm{h})\end{array}$ & $\begin{array}{c}\text { Methane } \\
(\text { SLPM) }\end{array}$ & $\begin{array}{c}\text { Oxygen } \\
(\text { SLPM) }\end{array}$ & $\begin{array}{c}\text { Argon } \\
(\text { SLPM })\end{array}$ & $\begin{array}{c}\text { Argon } \\
(\%)\end{array}$ & $\begin{array}{c}\text { Equivalence } \\
\text { Ratio }\end{array}$ & $\begin{array}{c}\text { Re } \\
\#\end{array}$ & $\begin{array}{c}\text { Flame } \\
(\text { Ref. \#) }\end{array}$ \\
\hline 20 & 408 & 1.20 & 13.20 & 4.0 & 70.1 & 1.16 & 675 & 11 \\
\hline 20 & 408 & 1.96 & 13.20 & 5.9 & 77.5 & 1.27 & 747 & 12 \\
\hline 20 & 408 & 1.20 & 17.16 & 4.0 & 70.1 & 0.889 & 795 & 13 \\
\hline 20 & 408 & 1.96 & 17.16 & 5.9 & 77.5 & 0.978 & 867 & 14 \\
\hline
\end{tabular}

\subsubsection{Fullerenes results}

The results of the fullerenes analysis from the turbulent flames is shown in Figures 3-23, 3-24, and 3-25. Figure 3-23 show the percentage of fullerenes $\left(\mathrm{C}_{60}+\mathrm{C}_{70}\right)$ in the condensable material, Figure 3-24 is the fullerene concentration in the cold sample gas, and Figure 3-25 shows the ratio of $\mathrm{C}_{60}$ to $\mathrm{C}_{70}$. In Figure 3-24, the data at the lowest height above burner has been omitted. Including its value of almost $1900 \mu \mathrm{g} / \mathrm{L}$ would have compressed the data from the other samples making it difficult to observe variation in the data. This point is most likely an outlier in any case. The data presented in Figures 3-23 to 3-25 are tabulated in Appendix 1.

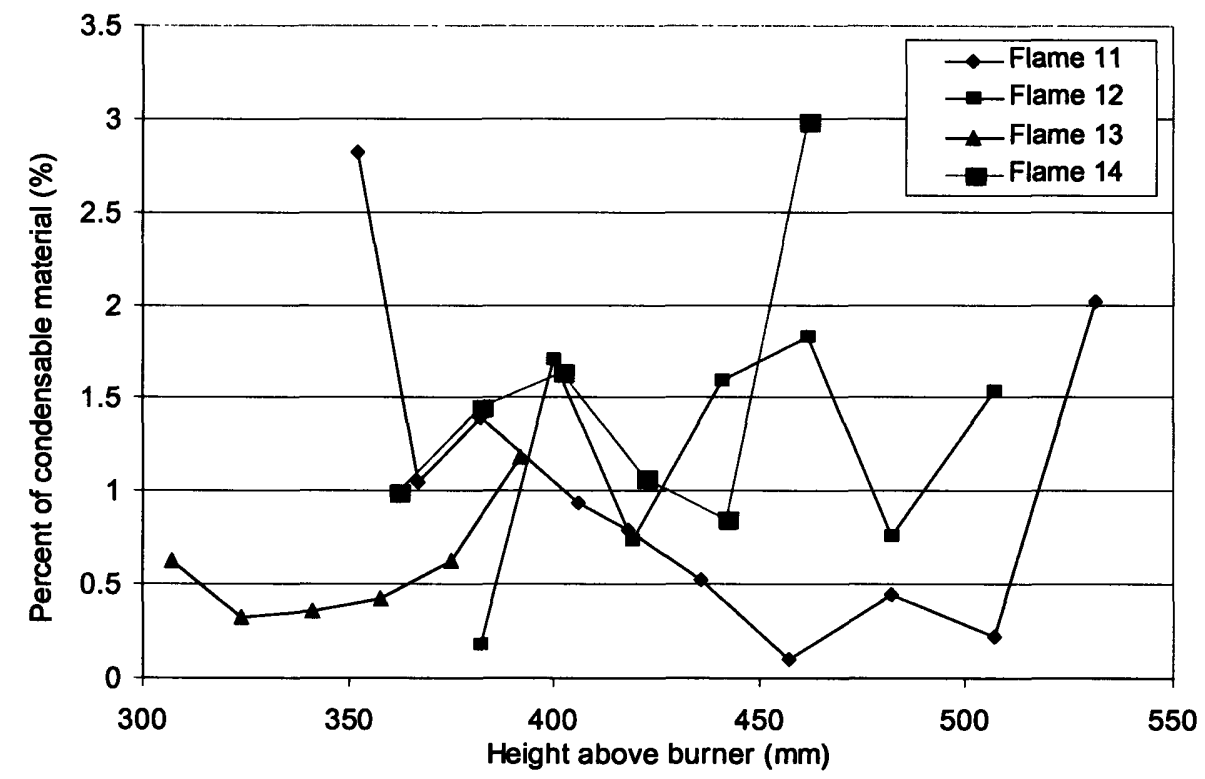

Figure 3-23. Quantity of fullerenes $C_{60}$ and $C_{70}$ in the condensable material from different heights above burner in different turbulent diffusion flames. 


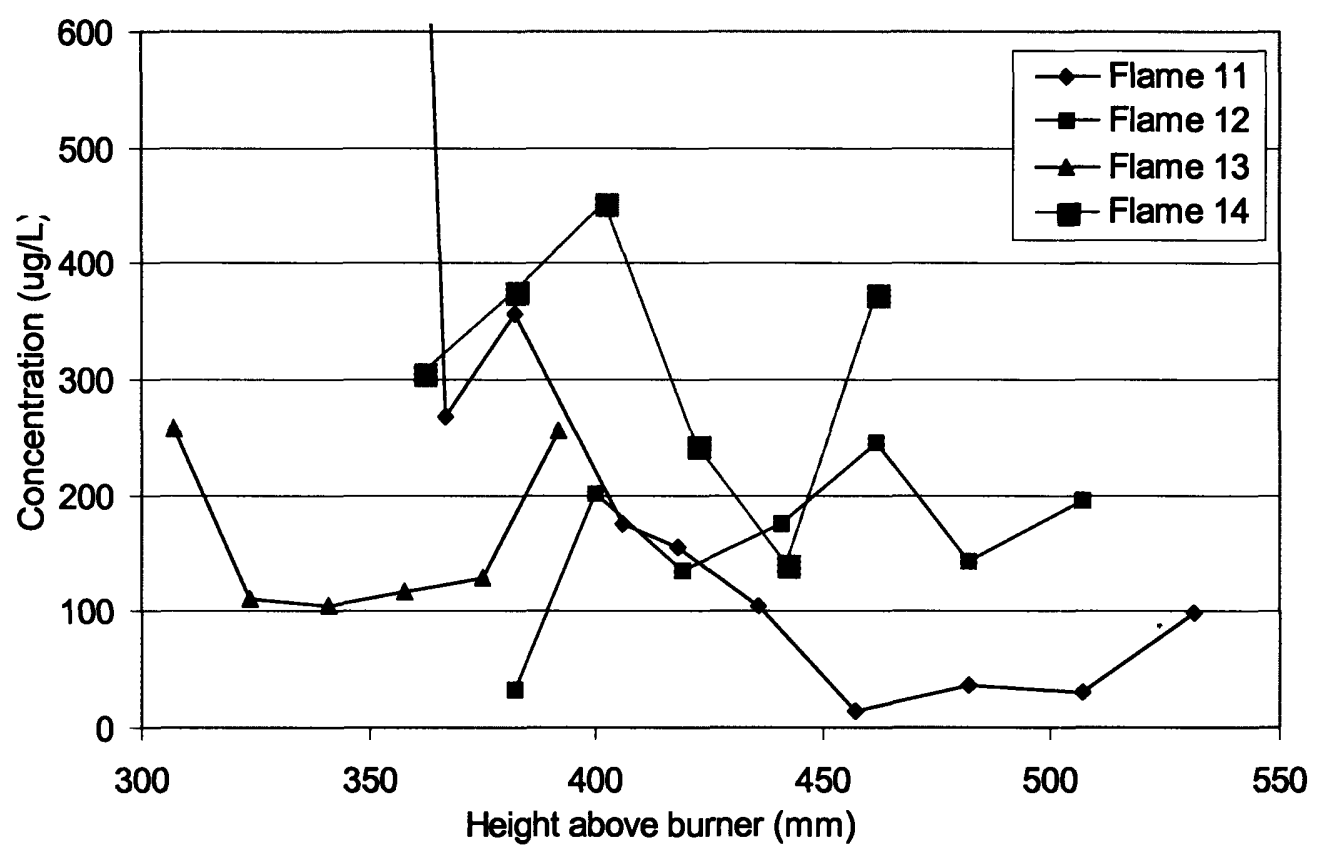

Figure 3-24. Concentration of fullerenes $C_{60}$ and $C_{70}$ in the cold probed gas from different heights above burner in different turbulent diffusion flames.

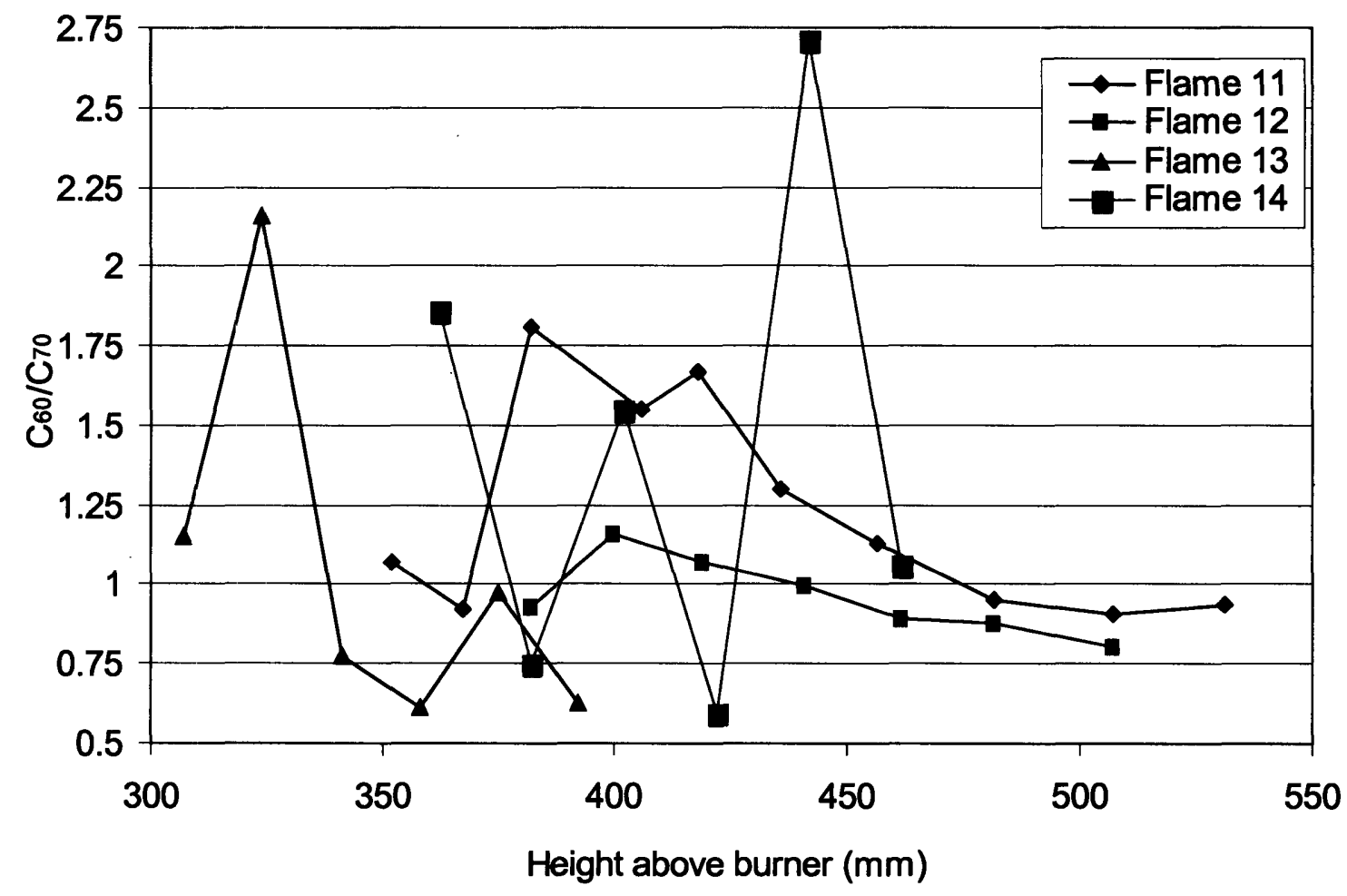

Figure 3-25. Mass ratio of $C_{60}$ to $C_{70}$ in the condensable material from different heights above burner in different turbulent diffusion flames. 
Figures 3-23 and 3-24 indicate that fullerenes are formed in turbulent diffusion flames and that they are formed in a rather large region of the flame, anywhere from 300 to $600 \mathrm{~mm}$ height above burner. However, the overall highest fullerene percentage recovered is only about $3 \%$ of the condensable material, with the vast majority of the data points falling below 2\% (Figure 3-23). Additionally, there is no clear trend in any of the data and there is an absence of a fullerene concentration maximum with increasing height above burner. In fact, Figures 3-23 through 3-25 show data that appear erratic and random, with some flames exhibiting points of minimum fullerene concentrations. Lastly, despite varying the oxygen and argon flowrates in the four flames, the data all lie at similar concentrations and heights above burner. Some sets of flame data even cross each other several times with increasing height. This indicates that the four flames all essentially have the same flame height and the same heat and mass transfer conditions. No fullerenic nanostructures were found in any of the HRTEM images taken from the samples of these flames.

\subsubsection{Discussion}

The presence of any fullerenes in the turbulent flames shows that production of fullerenes is possible in a wide range of diffusion flames and that they can be produced in turbulent as well as diffusion flames. This result alone is positive and promising for commercial production of fullerenes, which would most likely use a turbulent diffusion system. Another promising characteristic of the data is the production of fullerenes in a wide range of residence times, as measured by height above burner. Figure 3-23 shows that some flames (such as \#11) have a fullerene-producing region as wide as $200 \mathrm{~mm}$. Laminar diffusion flames show fullerene producing regions of a maximum of $20 \mathrm{~mm}$ (Figures 3-11 and 3-12). Hence, turbulent flames exhibit an order of magnitude increase in the region where fullerenes can be harvested. This observation also makes turbulent flames attractive for commercial production and even more so given that more heavily sooting conditions than those studied here would most likely give higher fullerene yields.

Possible explanations for the larger fullerene-producing region include the increased velocity of the fuel and oxygen and also the presence of flow eddies and vortices. The increase in velocity, independent of any other effects, would tend to 
increase the production region. If diffusion rates of oxygen and fuel are similar to those found in laminar flames, a reasonable assumption given that the pressures are similar, then fullerene formation should occur at similar residence times in both flames. Since higher gas velocities translate to a higher height above burner for a given residence time, it is not surprising that formation occurs at an increased range of heights. This explanation is supported by the fact that fullerene formation in turbulent flames occurs at heights entirely higher than in laminar flames $(>300 \mathrm{~mm}$ for turbulent vs. $<100$ for laminar - see Figures 3-11, 3-12, and 3-23). The flow eddies that were visually observable in the flames may also be contributing to the increased region of production. Any uncombusted fuel that is ejected out the side of the combustion zone (of which some was observed) can be caught by a vortex and reinjected into the flame region. This would bring fresh fuel into the flame and possibly create pockets of combustion that have conditions similar to those found closer to the burner. These pockets could then add some fullerene production downstream of where it would otherwise occur and extend the fullerene-producing region. Enough of these pockets of combustion aggregated together could significantly alter the fullerene concentration profile. Furthermore, flow vortices can help explain the erratic nature of the data as explained below.

Despite the confirmed presence of fullerenes and the increased region of production, it appears that turbulent flames under conditions studied here may not be so promising for commercial production. The primary reason is that the yields are much smaller than laminar flames in terms of fullerenes as a percentage of the total condensable material. Figures 3-11 through 3-13 show that laminar flames have yields consistently over $4 \%$ and up to $12 \%$ while turbulent flames have a maximum of $3 \%$ (Figure 3-23). Similar discrepancies are seen in terms of fullerene concentration in the cold sample gas. Figures 3-11 and 3-12 show gas concentrations greater than $1000 \mu \mathrm{g} / \mathrm{L}$ in general and up to $6000 \mu \mathrm{g} / \mathrm{L}$ for laminar flames while turbulent flames lag at less than $500 \mu \mathrm{g} / \mathrm{L}$ (Figure 3-24). This drop in yield may be the result of lower flame luminosity (indicating but not proving lower flame density), which was visually evident, or the flow eddies, which may pull more oxygen into the combustion zone and thus accelerate the consumption of the fullerenes. 
In addition to the low yields, the turbulent flame results are disappointing due to the absence of any fullerene maxima with respect to increasing height above burner or with respect to varying operating conditions. In contrast to the results for laminar flames, turbulent flames show no trends that would indicate the effect of residence time or operating parameters on fullerene synthesis. Again, the data sets seem quite erratic and even a bit random. The results of all four flames (Figure 3-23 and 3-24) lay on top of one another, cross each other on several occasions, and are clearly not separated as they are for laminar flames (Figures 3-11 through 3-13). One interpretation of this is that varying oxygen and diluent flowrates in turbulent flames has only limited effects on fullerene production. This is merely a postulation since only a small number of flames were tested but the results indicate that, if true, increasing fullerene yield in such types of flames will prove to be a difficult task.

The scatter in the data may be the result of the turbulent nature of the flames with the turbulence causing erratic and non-uniform flow patterns and concentrations that are reflected in the results. Consequently, reproduction of these results becomes unlikely and making predictions for other flame designs becomes highly speculative. Making accurate predictions and designing flames rich in fullerenes requires a detailed understanding of the effects of variations in operating conditions, which this data set unfortunately fails to provide.

The absence of any nanostructures in these turbulent flames is most likely the result of the general nature of turbulent flames and, more specifically, of the flow vortices. Nanostructures are generally found at longer residence times than fullerenes and the increased velocity of turbulent flames makes it difficult to take flame samples at suitable residence times. It is quite possible that nanostructures could be found at higher height above burner (HAB) than were studied here. Additionally, the flow vortices introduce and eliminate gases at all points along the flame changing effective residence times and preventing the accurate prediction of where nanostructures will occur. As mentioned above, the aggregation of the eddies could result in the significantly altered soot samples that lack nanostructures. 


\subsubsection{Summary}

The production of fullerenes in turbulent diffusion flames was tested in four different flame designs involving both high and low oxygen flowrates and high and low diluent flowrates. The results show that fullerenes can indeed be formed in such flames and that the fullerene-producing region is much greater than in laminar diffusion flames. However, the observed fullerene yields are significantly smaller than laminar flames and the data exhibit no detectable trends or maxima with respect to any parameter calling into question the presumed effect of changing the oxygen and diluent flowrates. It appears that even though turbulent flames can produce fullerenes, they are most likely unsuitable for commercial production as designing flames with higher yields based on the present data would be nearly impossible.

\subsection{Conclusions}

The study of diffusion flames for the production of fullerenes $\mathrm{C}_{60}$ and $\mathrm{C}_{70}$ has shown that while both laminar and turbulent flames are capable of making fullerenes, only laminar flames can make them in a predictable manner.

In laminar flames, the concentration of $\mathrm{C}_{60}$ and $\mathrm{C}_{70}$ increases with residence time until the point of fullerene maximum, which is just beyond the stoichiometric surface of the flame, after which it decreases. Curvature of carbon structures in the soot follows a similar pattern and nanostructures are found at much longer residence times than fullerenes. The fact the $\mathrm{C}_{60} / \mathrm{C}_{70}$ concentration declines after the stoichiometric surface, where soot production becomes prevalent, indicates that fullerenes may be consumed by soot in situ. Another possible explanation for the decline is oxidation, which may also be occurring. In terms of operating conditions, fullerene concentration goes through a peak with respect to increasing argon dilution, implying that increasing the dilution results in competing effects. Overall, shorter flames and flames that are hotter tend to have higher fullerene concentrations.

Turbulent flames do produce fullerenes but the increased gas flow velocity and the presence of flow vortices produce some unfavorable characteristics. The fullereneproducing region is larger than in laminar flames but the yields are much smaller and the data are erratic and unpredictable with respect to both residence time and operating 
conditions. Making predictions for productive turbulent flames from these data cannot be done with any accuracy.

It can be concluded that diffusion flames in general are promising for fullerene production given that fullerenes were found in every flame that was studied and the likelihood that diffusion flames are generally preferred for commercial production of materials. Laminar flames are more desirable due to their higher yields and distinct and predictable trends but turbulent flames would be easier for scale-up to commercial plant size. While this study accomplishes the thesis objectives for diffusion flames (Chapter 2), more study is needed to develop and optimize combustion systems for maximum fullerene production (Chapter 4). 


\section{$4 \quad$ Premixed flame studies}

Low-pressure premixed flames have been studied quite extensively in previous work and have identified a wide range of fullerenes and nanostructures in resulting condensable material [35-37, 85-88, 99]. However, results of recent studies on diffusion flames indicate that it may be possible to further increase the yield of premixed flames. In accordance, the previous studies were expanded to include conditions promising of high fullerene yields. These conditions consisted of two-stage flames with a premixed primary flame with varying equivalence rations and secondary feed. It was hypothesized that twostage flames would be highly fullerenic but the opposite was discovered to be the case.

\subsection{Apparatus and techniques}

The setup used for studying premixed flames was similar to that used in the diffusion flame study. Benzene was burned under sooting conditions to produce condensable material that was then analyzed for the presence and abundance of fullerenes. The fullerenes are extracted from the soot by sonication in toluene and then filtered before being injected into an HPLC instrument. Samples of the soot material were also analyzed by HRTEM prior to fullerene filtration.

\subsubsection{Flame chamber}

The premixed flame chamber, shown in Figure 4-1, is similar to the diffusion flame chamber discussed in the previous chapter. The premixed system, described previously $[99,117]$, consists of a burner mounted in the bottom of a cylindrical chamber housing that is sealed at the top. As this chamber is used only for laminar low-flow flames, which are generally shorter than diffusion flames, it has just one extension but does have a number of view- and auxiliary ports. Only the bottom section is shown in Figure 4-1. In this setup, the quartz sampling probe is inserted into the flame through the top plate, ensuring that flame sampling always occurs along the center axis of the flame.

The chamber is evacuated from the bottom with the same rotary vacuum pump as is used for the diffusion flame chamber (Mannesmann-Demag Model WPSO 53). The vacuum is maintained by bleeding air into the pumping system and is monitored by a pressure transducer (MKS Instruments Model 390HA-00100). 


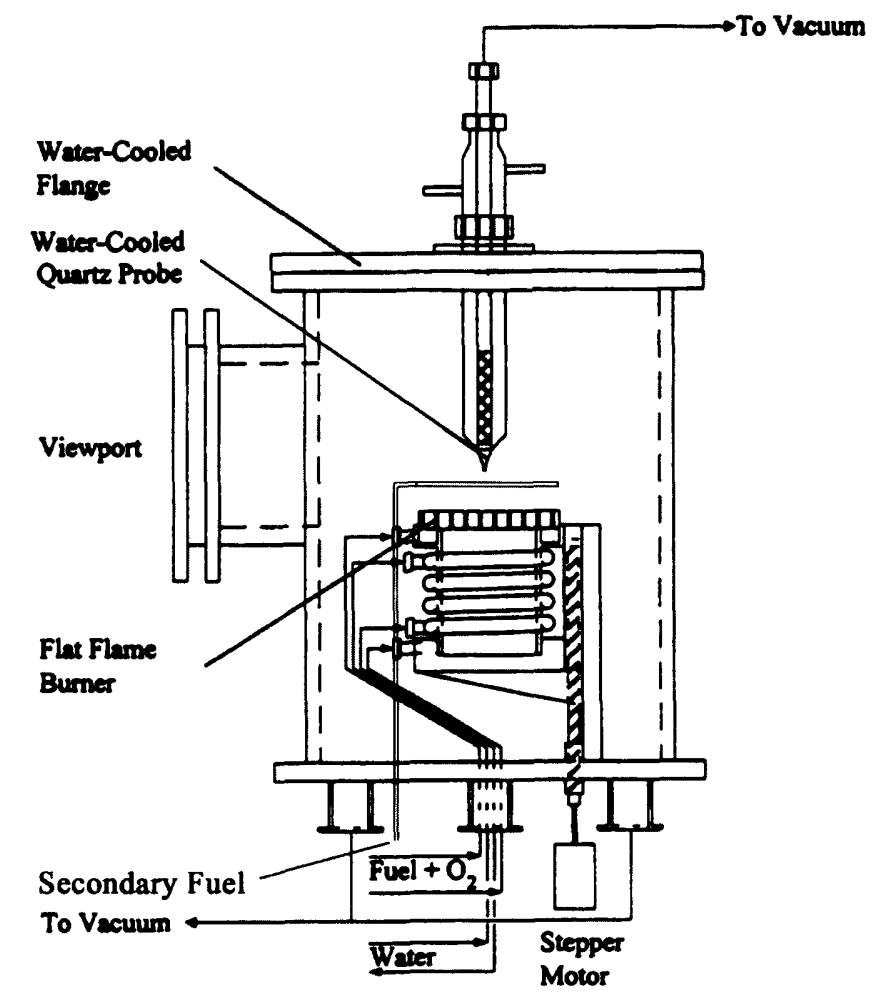

Figure 4-1. Premixed flame chamber with burner, top plate, secondary ring injector, and flame sampling probe (from Grieco [37]).

\subsubsection{Flame burner}

The burner, shown up close in Figure 4-2 with an accompanying top-view, is a premixed one-dimensional laminar flat flame burner. It is water-cooled to prevent melting and consists of a thick copper plate ( $100 \mathrm{~mm}$ diameter $\mathrm{x} 12 \mathrm{~mm})$ mounted on top of a cylindrical body through which the gases are fed. The plate is drilled with approximately $15001.0 \mathrm{~mm}$ holes through which the fuel, oxygen, and diluent flow. As seen at the top of Figure 4-2, the burner is divided into a 70-mm diameter core, where the main flame is stabilized, and a 15-mm wide annulus, which supports a non-sooting premixed thermally-shielding ethylene/oxygen flame.

The body of the burner is packed with stainless steel wool to quench any flashback of the flame and to distribute the gas flow uniformly across the diameter. The entire assembly is mounted on a vertical translation stage controlled with a stepper motor, mounted and controlled from outside the chamber. The motor allows the burner height to vary during a single flame run and also allows for a greater range of distance from the sampling probe. More importantly, since the changes in the height of the burner can be 
measured more accurately than those of the probe, moving the burner vertically instead of the probe results in a higher resolution of flame data.

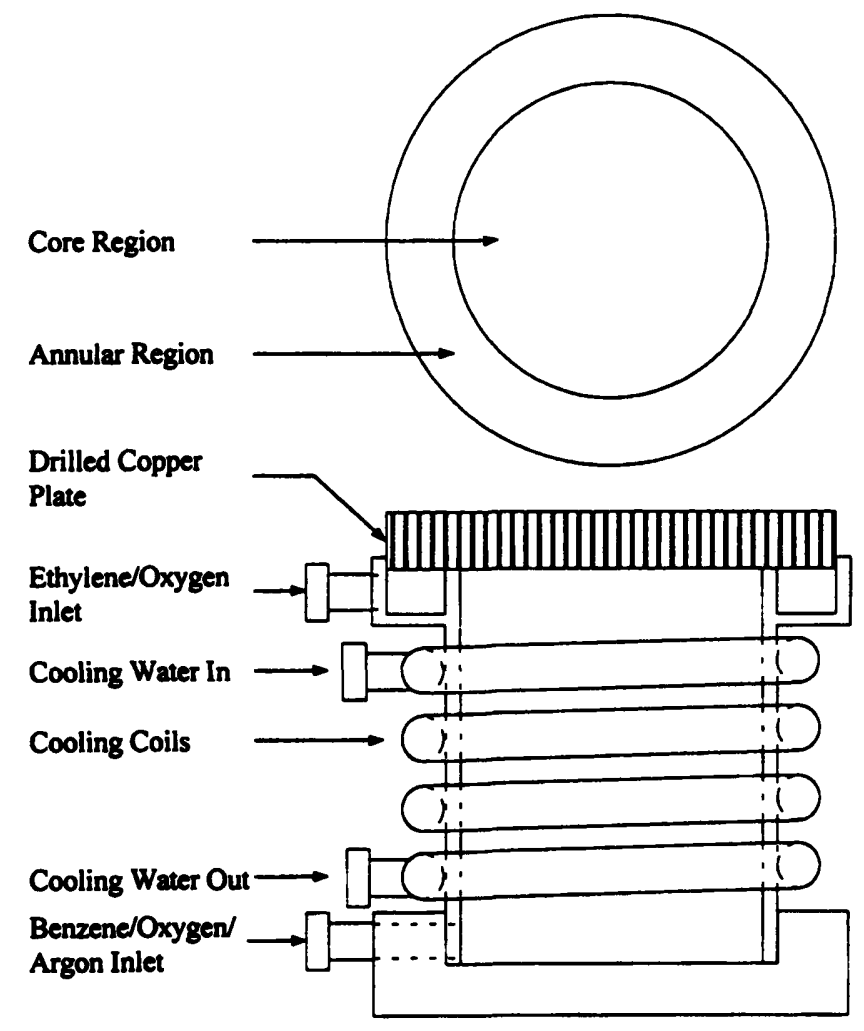

Figure 4-2. Premixed flame burner with top view (from Grieco [37]).

For two-stage flames, a secondary ring injector was mounted inside the chamber. The location and setup of the ring injector can be seen in Figure 4-1. The injector consists of a stainless steel tubular ring with injector holes that is supported by a stainless steel supply tube through which the secondary fuel is fed to the system. The supply tube passes through a feed port in the bottom of the chamber and can be adjusted vertically to allow for secondary injection at varying height above the burner.

In the two-stage flames studied here, two different ring injectors were used.

Figure 4-3 shows top views of both rings to highlight the differences. The large injector, Figure 4-3(a), has an inner diameter of $100 \mathrm{~mm}$ and contains four outlet holes that are evenly distributed around the inside of the ring and used for fuel injection. The small injector, Figure 4-3(b), has an inner diameter of $50 \mathrm{~mm}$ and has eight holes distributed about both sides of the ring. The holes in both rings have a diameter of 1/16 in. The larger ring has the advantage of disturbing the primary flame only minimally, if at all, as the 
ring is not immersed in the flame, but the smaller ring provides a more evenly distributed secondary fuel flow due to its greater number of injection holes and their configuration.

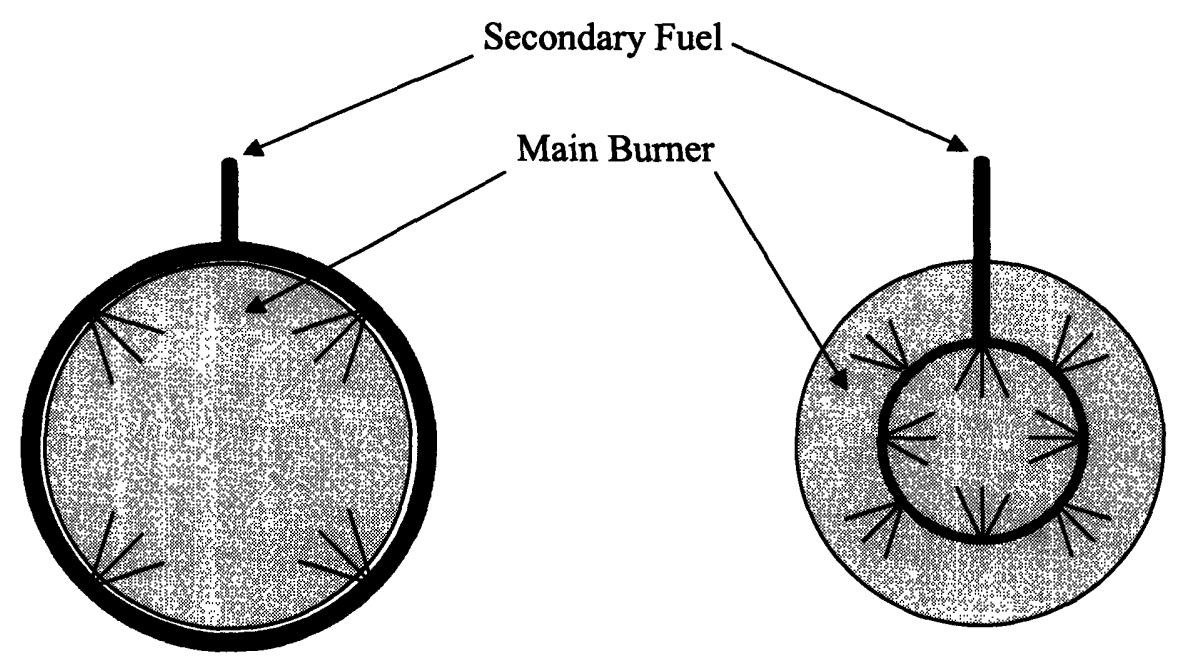

(a)

(b)

Figure 4-3. Top view of large (a) and small (b) secondary ring injectors

\subsubsection{Chamber bottom and extension}

The chamber consists of two sections, the top section of which is removable. Both sections are made from stainless steel, have inner diameters of $24 \mathrm{~cm}$ and have a combined height of 25 in. allowing flames up to $18 \mathrm{in}$. in height to be operated (the sum of the chambers less the burner height). The bottom section is already shown in Figure 41 and the top section is shown in Figure 4-4.

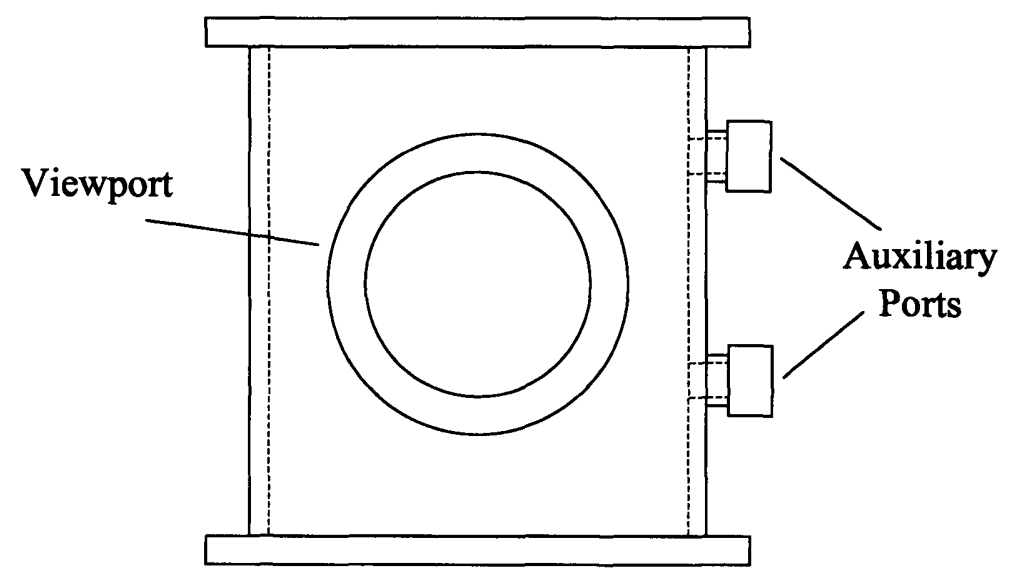

Figure 4-4. Top section of the premixed flame chamber. 
The bottom chamber has one viewport of 6 inch in diameter, and six smaller auxiliary ports, three with diameters of one inch and three with diameters of two inches (not shown in Figure 4-1). The auxiliary ports can be adapted to support feedthrough lines, thermocouples, transducers, and even optical windows for laser scatter or optical pyrometry. The bottom plate seals the chamber, has two $1 \frac{1}{2} \mathrm{in}$. diameter ports for chamber evacuation, and has multiple feedthroughs for the burner. The top section is nearly identical to the bottom, the only exception being fewer auxiliary ports. Again there is a large 6 inch diameter viewport and two one-inch auxiliary ports for various uses.

\subsubsection{Top plate}

The top plate for the premixed chamber is identical to the one used in the diffusion chamber and is thus not shown again here (see Figure 3-8). The only difference between the two is the use of the ports - the premixed chamber is evacuated from the bottom meaning that the two larger ports on the top plate are sealed off.

\subsubsection{Fuel delivery}

Vaporized benzene was the sole fuel used in the premixed flame burner. See Section 3.1.2 for a description of the vaporizer used in these experiments. All the flows (argon, oxygen, ethylene, acetylene) are controlled with critical orifices and upstream metering valves. Here, the pressures are monitored with similar Omega engineering pressure transducers.

\subsubsection{Soot sampling}

The quartz probing system described in Section 3.1.3 was used here with the probe entering from the top (Figure 4-1). In addition to samples obtained from the quartz probe, condensable material was collected after a flame had been extinguished from deposits on the chamber walls and the top plate. These deposits were sampled simply by scraping the surfaces with a spatula and collecting the material into a preweighed vial. More condensable material deposits on the top plate than on the chamber walls. This is because the top plate is water-cooled and the flame gases flow closer to the top plate than the chamber walls. The soot collected in this manner was most in providing material for the heat treatment experiments that are described in the next chapter. 


\subsubsection{Extraction and analysis}

The method of fullerene extraction and HPLC analysis was identical to that used in the diffusion flame studies. The details are provided in Sections 3.1.4 and 3.1.5 and not reproduced here. Qualitative analysis of soot material with HRTEM images was also performed on premixed two-stage flame soot material.

\subsection{Premixed flames}

Before beginning investigation into two-stage flames, a single-stage premixed flame was studied. This flame has previously been studied extensively and the objectives here were two-fold: reproduce earlier data and ensure the system is working properly; and provide a baseline to compare data taken under altered two-stage conditions. The flame was analyzed for fullerene concentration by HPLC; HRTEM analysis was not performed.

\subsubsection{Experimental conditions}

The baseline flame consisted of pure benzene fuel mixed with oxygen and argon diluent. The secondary ring injector was not used for this flame and hence, was removed from the system. The operating conditions of the flame were as follows: equivalence ratio, 2.4; cold gas velocity, $25 \mathrm{~cm} / \mathrm{s}$; pressure, 40 torr; argon diluent, $10 \mathrm{vol} \%$. This flame has been well-characterized and identified as fullerene forming [35-37, 85-88, 99]. The flame was designed by Tom McKinnon [117] and is often referred to as the McKinnon flame, as it will be called in the remainder of this chapter.

\subsubsection{Fullerene results}

Consistent with previous studies, the McKinnon flame was found to be very fullerenic with the concentration of fullerenes increasing with residence time. A plot of the $\mathrm{C}_{60}$ and $\mathrm{C}_{70}$ data is shown in Figure 4-5 and a plot of the soot data is shown in Figure 4-6. These data sets and a sample calculation are documented in Appendix 2.

It can be seen in Figure 4-5 that $\mathrm{C}_{60}$ and $\mathrm{C}_{70}$ concentrations follow similar trends. They are quite low in the primary flame zone $(5-20 \mathrm{~mm})$ but increase steadily with residence time above $20 \mathrm{~mm}$. A couple of oscillations are seen in the data as the concentration increases. This is most notable at 50 and $80-90 \mathrm{~mm}$ HAB. No data could be 
collected above $120 \mathrm{~mm} \mathrm{HAB}$ as the limit of the extended combustion chamber had been reached. It should be noted that even at the longest residence time studied here, a drop-off in the fullerene concentration was not observed. It is believed that measurements at higher $\mathrm{HAB}$ would result in similar increases in concentration due to cooling of the flame gases.

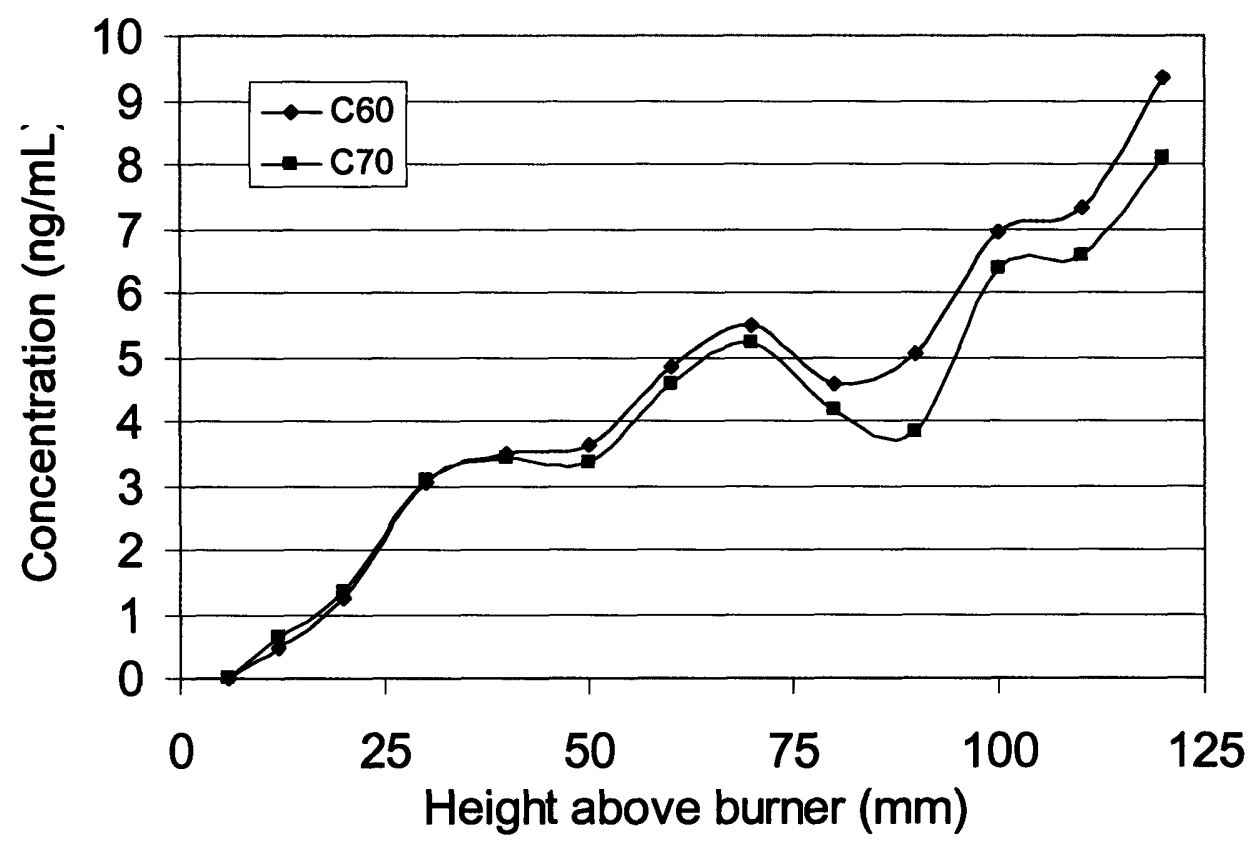

Figure 4-5. Concentration of $C_{60}$ and $C_{70}$ in a premixed benzene flame ( $p=40$ torr, $\varphi=$ 2.4 , velocity $=25 \mathrm{~cm} / \mathrm{s}$, dilution $=10 \%$ )

In Figure 4-6, it is seen that the soot concentration increases steadily with increasing residence time (with the exception of oscillations at $50 \mathrm{~mm}$ and $80 \mathrm{~mm} \mathrm{HAB}$ ). The oscillations coincide exactly with the oscillations in fullerene concentration seen in Figure 4-5 and could possible be related. Again, no drop-off in soot concentration was observed even at the longest residence time studied here.

Fullerene and soot data from Grieco's study of the same flame [89] are reproduced in Figures 4-7 and 4-8 for comparison to the present data. Figure 4-7 shows fullerene concentration against $\mathrm{HAB}$ while Figure 4-8 shows soot data, represented by DCM insoluble material, over the same region. In Figure 4-7, once can see a marked oscillation at $50 \mathrm{~mm}$ and a drop-off in fullerene concentration at $78 \mathrm{~mm}$. The data in Figure 4-8 indicate that soot concentration is low in the primary flame zone but 
significantly increases thereafter. The trend is almost exactly similar to that of fullerene concentration (Figure 4-7). The DCM soluble material in Figure 4-8 represents the sum of PAH, fullerenes, and other light gas species and is not important in the present study.

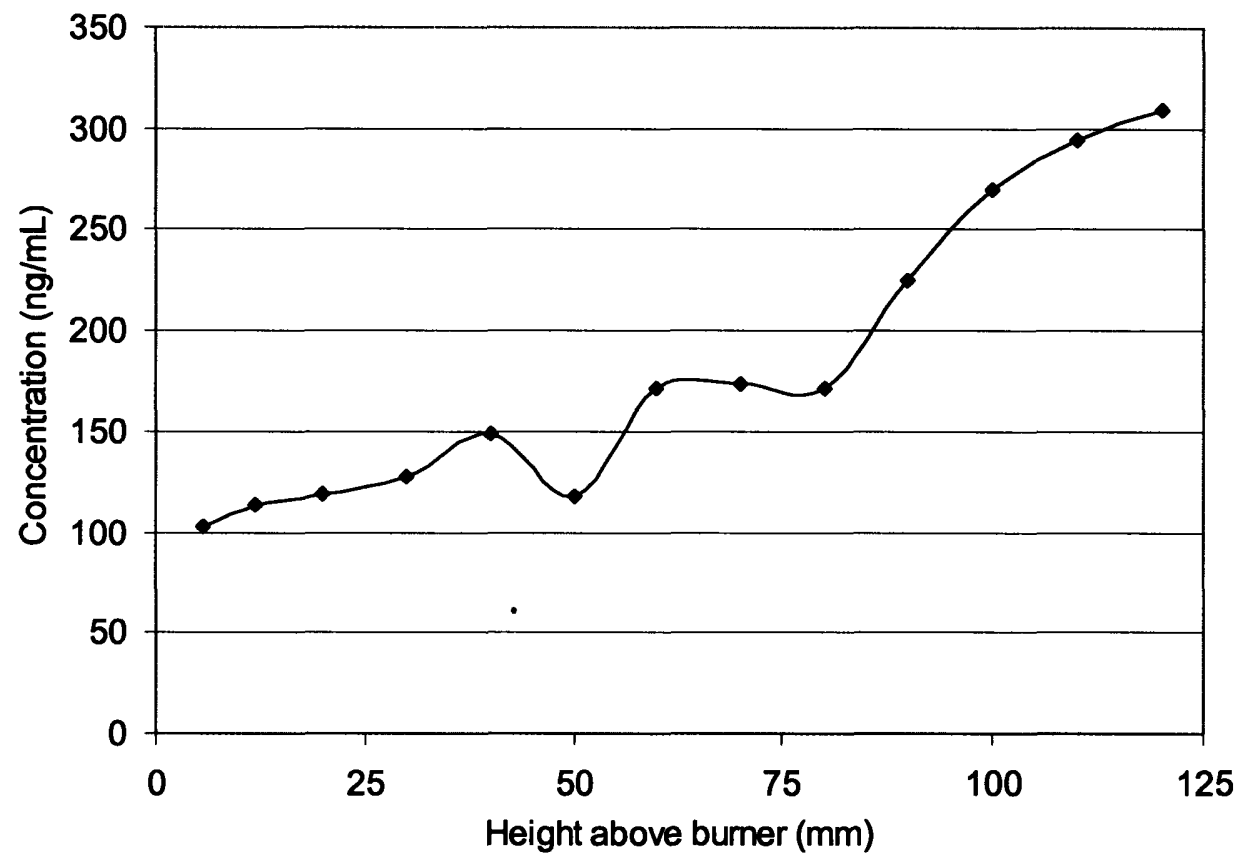

Figure 4-6. Concentration of soot in a premixed benzene flame ( $p=40$ torr, $\varphi=2.4$, velocity $=25 \mathrm{~cm} / \mathrm{s}$, dilution $=10 \%$ )

Comparison of Figure 4-5 with Figure 4-7 shows that the data from this experiment are greater than previous data by approximately a factor of two at all HAB along the flame and that there is no fullerene drop-off above $70 \mathrm{~mm}$ as does the previous data. In fact, the fullerene concentration keeps increasing until the limit of soot collection capabilities is reached.

By comparing Figure 4-6 with Figure 4-8, it can be seen that the present soot data are also greater than the previous soot data, but the difference here is approximately a factor of six at all HAB. Similar to the fullerene data, the present soot data show an oscillation at $50 \mathrm{~mm}$ but this data point is conspicuously absent in Figure 4-8, preventing a comparison. The soot concentration also continued to increase above $70 \mathrm{~mm}$ with another oscillation at $80 \mathrm{~mm}$. 


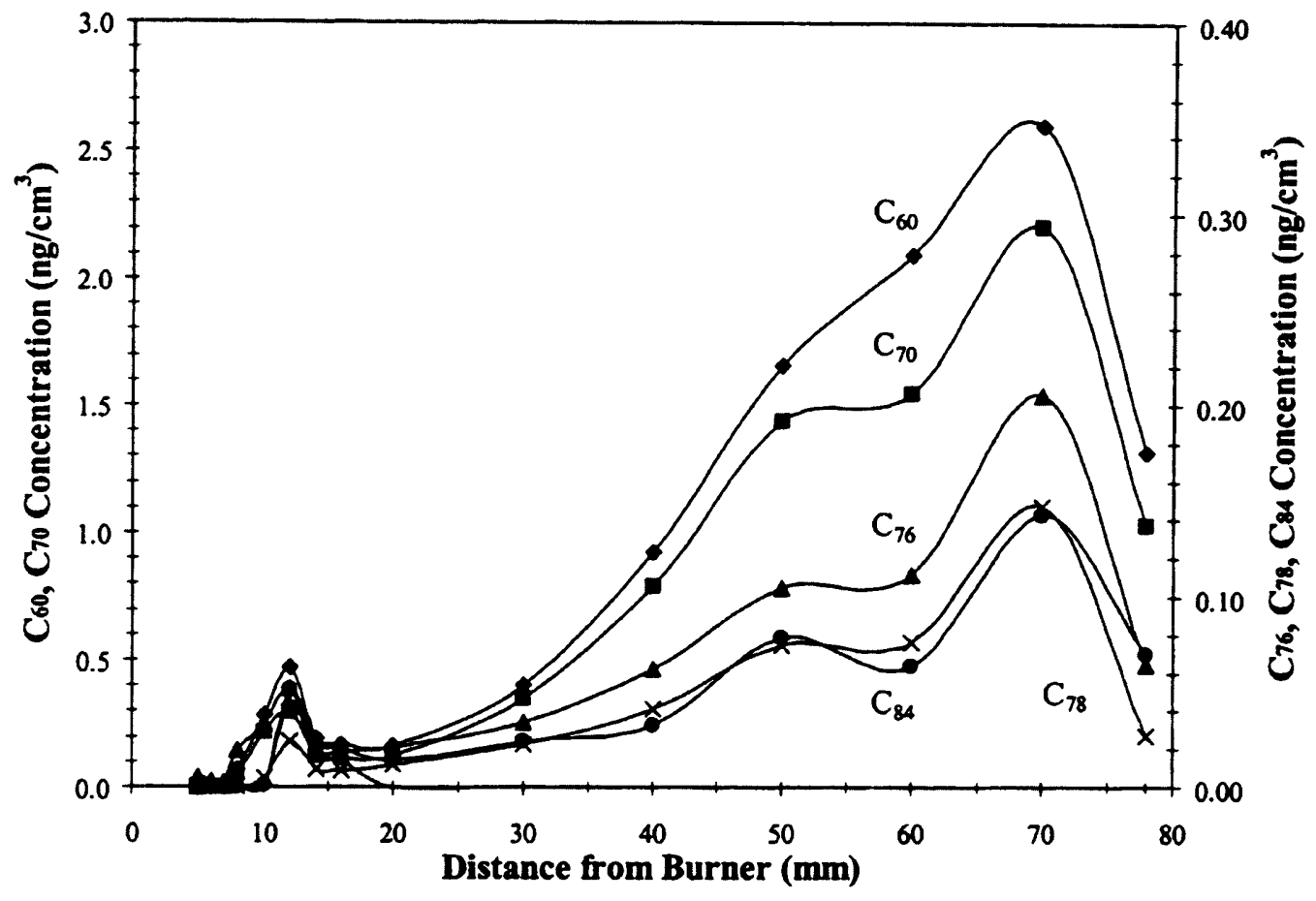

Figure 4-7. Concentration of fullerenes in a premixed benzene flame ( $p=40$ torr, $\varphi=$ 2.4 , velocity $=25 \mathrm{~cm} / \mathrm{s}$, dilution $=10 \%$ ) [89]

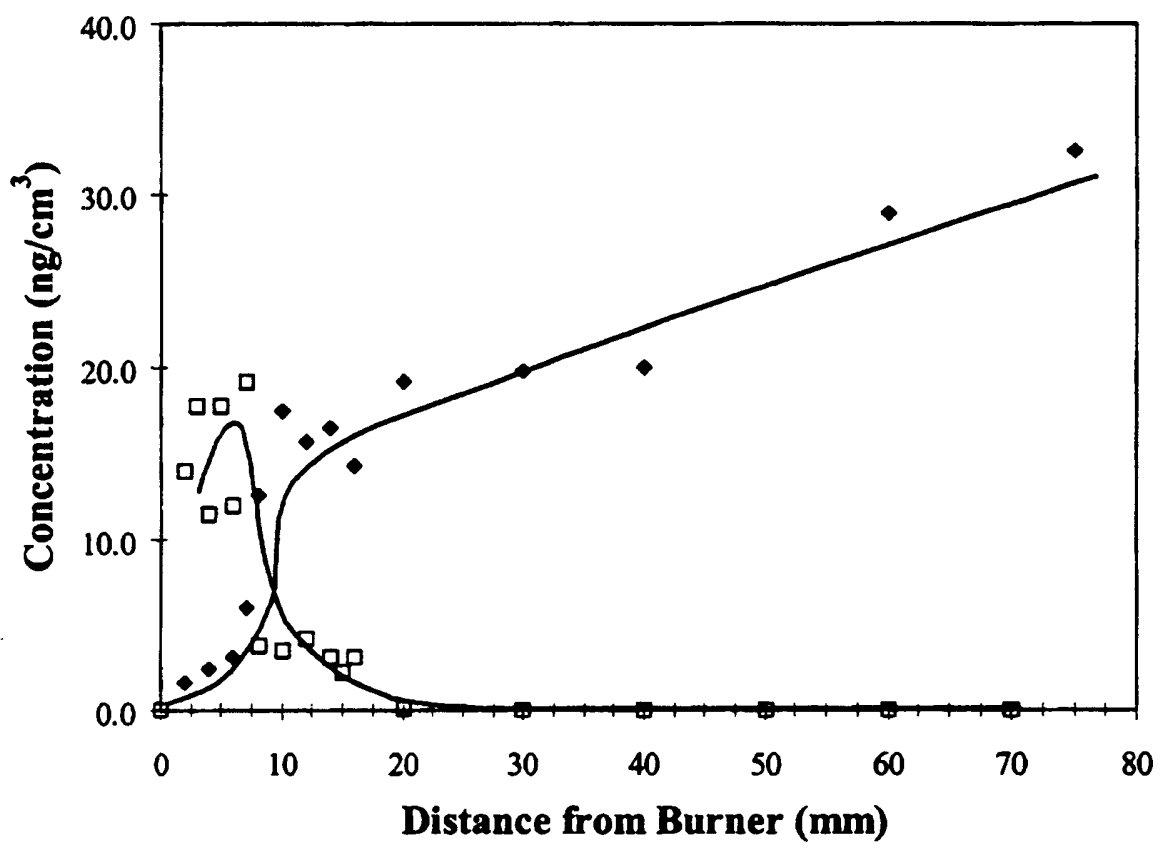

Figure 4-8. Concentration of DCM soluble material () and DCM insoluble material ( $\square$ ) in a premixed benzene flame ( $p=40$ torr, $\varphi=2.4$, velocity $=25 \mathrm{~cm} / \mathrm{s}$, dilution $=10 \%$ ) 
All data sets, however, exhibit similar trends up until $70 \mathrm{~mm}$ and the concentration oscillations coincide at $50 \mathrm{~mm}$. In fact, the similarity of the data otherwise is what would be expected for data that are collected from what are believed to be identical flames.

If Figure 4-5 is compared to the soot data in Figure 4-6, it becomes evident that significant fullerene production coincides directly with the increase in soot material. Fullerene concentration is almost insignificant below $20 \mathrm{~mm}$, which is the exact region where soot is relatively low. Beyond $20 \mathrm{~mm}$, where the primary flame reactions zone ends, a steep rise in soot concentration is observed and correspondingly, a rise in fullerenic material is seen. The increase in both materials continues until the limit of the flame data, i.e. the apparatus, is reached.

\subsubsection{Discussion}

As noted above, the major differences between the data from this experiment and the previous data are the disagreement in the magnitude of the fullerene concentration and the absence of a concentration drop-off above $70 \mathrm{~mm}$ in the present data. It was expected that reproducing the McKinnon flame would produce results that were in better agreement with previous data. The question then arises: what is causing the discrepancy. Why are the two data sets different when the flames are supposedly identical?

Although the true cause is still unknown, several possible explanations for this inconsistency can be stipulated. The first is that in the previous study, Grieco used a macroprobe sampling system [37], while a microprobe sample system described in Section 3.1.3 was used here. Although Grieco claims that the differences between the two sampling systems are negligible, there are important structural differences that can lead to inconsistent data. The macroprobe system is used in place of the chamber top plate. This means that the flame is significantly disturbed by the intrusion of the 'massive' probe and an obstruction just downstream of the sampling location. In contrast, the microprobe is relatively unintrusive and allows the flame gases to flow beyond the probe until they reach the top plate, which is significantly downstream. Additionally, since the macroprobe is part of the top plate, the extension must be moved to sample at any of the points that Grieco measured, meaning that the top plate could be quenching or otherwise 
affecting the flame reactions. Moving the microprobe, on the other hand, ensures that any top plate effects are always well downstream of the sample location. A disturbance in the flame such as the macroprobe could cause suppressed fullerene production as well as a significant unexpected drop-off.

Another explanation is the fact that different benzene vaporizer systems were used to deliver fuel to the chamber and burner. Grieco used an old vaporizer system that was rather unstable [118]. It consisted of a syringe pump delivering liquid fuel to a heating element that provided a continuous evaporation of the flowing benzene. Theoretically, the mass flow delivered to the burner was identical to that set on the syringe pump but visual observations showed that the flame was unstable and pulsated with variable fuel flow even several hours after start-up [118]. The system used here, described in Section 3.1.2, used a mass flow controller to meter in benzene that had been vaporized upstream of it. This system provides an accurate and steady flow for the duration of all experiments. No instability or strobing was observed in the present study. Although it is difficult to explain exactly how such a difference in delivery systems could result in the observed data discrepancy, it is reasonable to assume that this inconsistency may somehow be attributed to differences in the flames that are caused by their respective fuel vaporizers.

Despite this discrepancy, the data from the two flames are similar enough to conclude that the flame has effectively been reproduced and that a true McKinnon flame was studied here. It should be kept in mind that the differences in the data are only a factor of two, not an order of magnitude, and that the trends and oscillations exactly coincide until $70 \mathrm{~mm}$, which is beyond the primary region of interest. The results of the McKinnon flame have provided a reasonable baseline for which to compare two-stage flame data outlined in the next section.

The coincidence of the fullerene trend (Figure 4-5) and the soot trend (Figure 4-6) is interesting as it contradicts the assertion from diffusion flame data that soot consumes the fullerenes (Section 3.4). If Grieco's data are to be believed (Figure 4-7), then the decline between 70 and $78 \mathrm{~mm}$ could be attributed to consumption by soot which is consistent with the diffusion flame conclusion. However, the current data exhibits no such decline (neither does Grieco's data lower than $70 \mathrm{~mm} \mathrm{HAB}$ ), which is inconsistent 
with the hypothesis of fullerene consumption by soot. This could be explained by assuming that fullerene concentration is a net result of consumption and formation reactions and that the formation reactions are fast enough to overcome the consumption in the McKinnon flame but not so in the diffusion flames studied. Still, the observations in the McKinnon flame cast doubt on the consumption of fullerenes by soot mechanism. This doubt was part of the motivation for the more detailed study of the fullerene-soot reaction in Chapter 5.

\subsubsection{Summary}

The data from the McKinnon flame study were relatively consistent with those from a previous study by Grieco. A few discrepancies between the two data sets did arise but the major trends in the data are similar (within an order of magnitude) indicating that the flame has been reproduced. The current data provides a baseline to which two-stage flame data can be compared. Comparison of the fullerene data with previously collected soot concentration data indicated that fullerenes might not be reacting with the soot in a manner that consumes the fullerenes as was asserted by the diffusion flame data. At this point, no suitable explanation can be found for the inconsistency but the matter provides motivation for the fullerene-soot reaction study later in the thesis. The acquisition of new McKinnon flame data allows for further premixed flame study, which is presented in the remainder of this chapter.

\subsection{Premixed flames with secondary injections}

Benzene is used as the primary fuel here because, among the tested organic solvents, it provides the highest yield of fullerene material $[32,33]$. However, much of the benzene is used in providing energy for the combustion process instead of for producing fullerenes. It may be possible to use a different, perhaps cheaper, fuel for the energy supply than the benzene needed for product formation. Separating the combustion process from the fullerene production process (in a two-stage flame) has the potential to greatly increase the overall fullerene yield and also reduce costs. More carbon material may be consumed in the two fuel feeds but it is believed that a greater fullerene production will offset this consumption. This type of premixed combustion system had not been previously studied. 


\subsubsection{Experimental conditions}

The two-stage flames studied here utilized the secondary fuel ring injectors described in Section 4.1.1.1, which were positioned at various heights above burner for different flames. The location of the secondary fuel injection was always downstream of the primary flame reaction zone so as to only minimally disturb the primary flame.

Flames with both benzene and acetylene as secondary fuels were studied. Not only was the flowrate of the secondary fuel varied but the equivalence ratio of the primary flame was also varied to provide both sooting and non-sooting primary flames. All of the flames, however used the McKinnon flame as a baseline and incorporated variations to it involving the secondary injection. This means the pressure was held constant at 40 Torr and the argon flowrate was also held constant for all the flames.

Six different two-stage flames were studied. Their operating conditions are summarized in Table 4-1.

Table 4-1. Two-stage flame parameters.

\begin{tabular}{|c|l|l|l|c|c|}
\hline$\#$ & Secondary fuel & Location & Difference from McKinnon & Primary $\varphi$ & Total $\varphi$ \\
\hline 1 & Benzene & $35 \mathrm{~mm}$ & $25 \%$ more fuel & 2.4 & 3.0 \\
\hline 2 & Benzene & $35 \mathrm{~mm}$ & $25 \%$ rerouted & 1.8 & 2.4 \\
\hline 3 & Benzene & $15 \mathrm{~mm}$ & $43 \%$ rerouted & 1.37 & 2.4 \\
\hline 4 & Acetylene & $35 \mathrm{~mm}$ & $11 \%$ more fuel & 2.4 & 2.66 \\
\hline 5 & Acetylene & $35 \mathrm{~mm}$ & $25 \%$ replaced with $22 \%$ & 1.97 & 2.4 \\
\hline 6 & Acetylene & $35 \mathrm{~mm}$ & $25 \%$ replaced with $22 \%$ - small ring & 1.97 & 2.4 \\
\hline
\end{tabular}

In Table 4-1, the number in the first column is used for flame identification in the results in the next section. The 'Difference from McKinnon' documents explains how the McKinnon flame was altered to achieve that two-stage flame. For example, in flames 1 and 4, additional fuel was added beyond that used for the McKinnon flame while in the others, some of the McKinnon fuel was replaced by the secondary fuel. In flames 5 and 6 , $25 \%$ of the primary fuel was removed but only $22 \%$ was reinjected in the secondary feed. Note that flame 6 was the only one to use the small ring secondary fuel injector. The last 
two columns respectively give the equivalence ratio of the primary flame only and the overall equivalence ratio. The percentages given in Table 4-1 represent the percentages of carbon atoms being fed that were altered. The volume percentage changes for the benzene two-stage flames would be similar but those for acetylene would be tripled as one molecule of acetylene contains only $1 / 3$ as much carbon as one of benzene.

The collected sample material was analyzed by HPLC for fullerene concentration and the HPLC chromatograms were used to compare soot across different flames and make inferences as to the conditions present at the locations that those soot samples were taken. This chromatogram analysis provided another basis for comparing the results in additions to the $\mathrm{C}_{60}$ concentration. Analysis by HRTEM was also performed on selected soot samples.

\subsubsection{Fullerene Results}

The data from the six two-stage flames are shown in Figures 4-9 and 4-10. To avoid clutter, the data were split into two sets, and lines for the data sets were omitted. The $\mathrm{C}_{70}$ data, which follows the same trend as the $\mathrm{C}_{60}$ data, was also omitted. Figure 4-9 shows the results of the three secondary benzene flames while Figure 4-10 shows the three secondary acetylene flames (all tabulated in Appendix 2). In each plot, the $\mathrm{C}_{60}$ data from the regular McKinnon flame (Figure 4-5) is shown for comparison.

In Figure 4-9, it can be seen that the two-stage flames are very poor producers of fullerenes. The data, which begin at the point of secondary fuel injection, show a significant decrease in (flame \#1) or complete absence of (flames \#2 and \#3) fullerene concentration. It should be noted that the only two-stage flame produce fullerenes at all was \#1, which was the only one of these three to have a sooting primary flame. Still, even in flame \#1, while the fullerene concentration is comparable to the McKinnon flame at the location of secondary injection, it subsequently drops drastically and does not recover until much later in the flame. The other two flames do not even show a hint of fullerene production anywhere in the flame. 


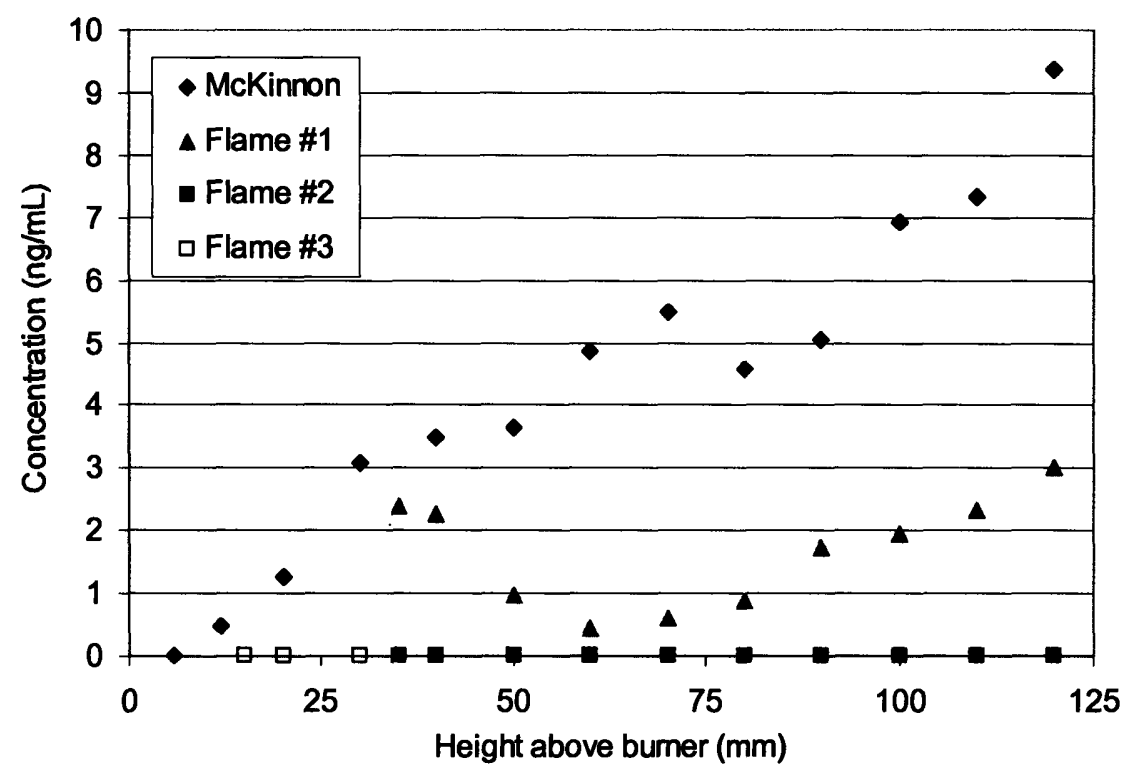

Figure 4-9. Flame gas concentration of $C_{60}$ in McKinnon flame and three two-stage flames with benzene as secondary fuel.

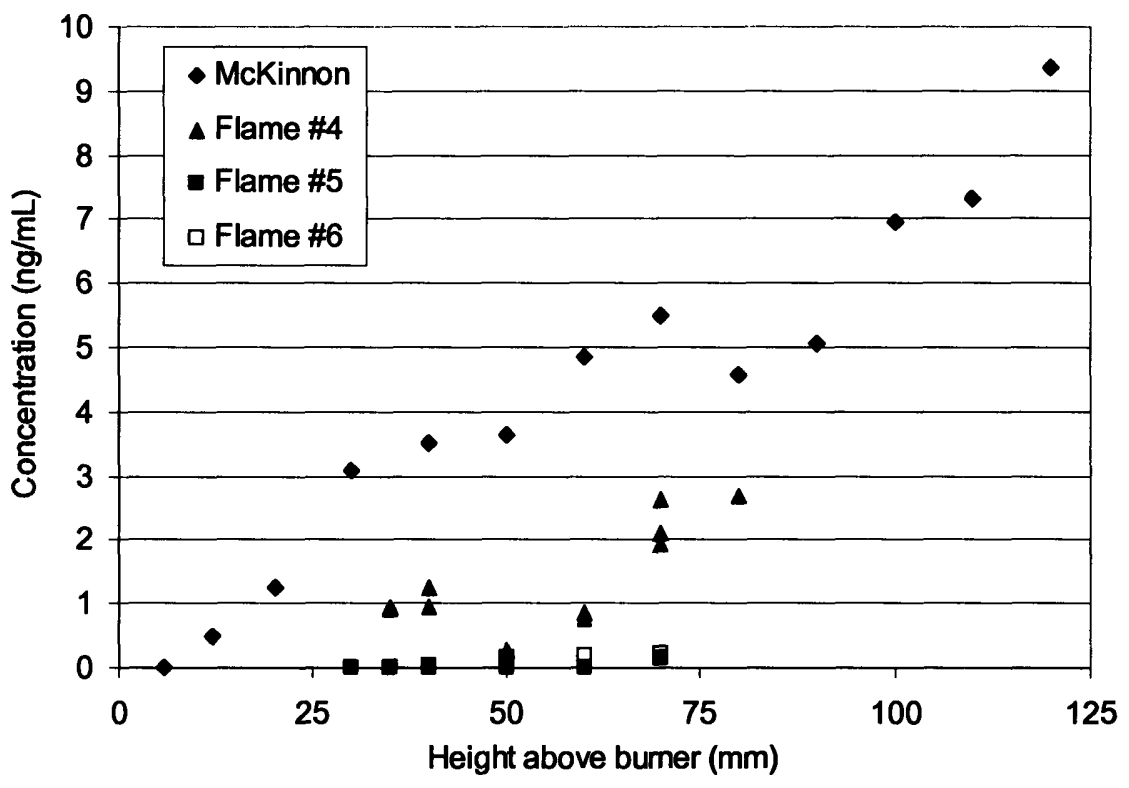

Figure 4-10. Flame gas concentration of $C_{60}$ in McKinnon flame and three two-stage flames with acetylene as secondary fuel.

Similar observations can be made for the three secondary acetylene flames shown in Figure 4-10. Flame \#4, which was the only with a sooting primary flame, also was the only one to show a significant amount of fullerene production. Again, though, the concentration levels are well below those observed in the McKinnon flame and begin to 
recover only downstream. The data in flame \#4 also show a more immediate effect of the secondary injection. Note that the concentration at $35 \mathrm{~mm} \mathrm{HAB}$, the location of the secondary injection, is already a fraction of the McKinnon flame's. Flames \#5 and \#6 do show some traces of fullerene formation at $50 \mathrm{~mm} \mathrm{HAB}$ and higher but these concentration levels are negligible and not interesting enough to warrant further characterization of the flames.

In fact, characterization of flames \#3-\#6 above $60-80 \mathrm{~mm}$ was not performed as it was believed that no more interesting data would be collected at these heights. Flame \#2 data provided uninteresting data at these heights and as such were used as justification for abandoning Flames \#3, \#5, and \#6. Flame \#4 could have been interesting at higher HAB but the small soot yields (and the disappointment in Flame \#1 results) precluded any interest in further characterizing that flame.

Qualitative HRTEM analysis was performed on three selected samples from the two-stage flames. However, no nanostructures or other interesting structures were found and thus, quantitative analysis was not performed.

\subsubsection{Discussion}

After examining all of the two-stage flame data collectively, it seems that secondary fuel has an adverse effect on the formation of fullerenes. Results from both the secondary benzene and secondary acetylene flames were quite similar and they indicate that fullerenes are formed only when the primary flame is sooting. It was seen that the injection of secondary fuel actually caused a decrease in fullerene concentration subsequent to the location of injection and that fullerene production recovered only at much higher $\mathrm{HAB}$, if at all.

The fact that only flames with sooting primary flames produced fullerenes at all indicates that the combustion process is necessary for making fullerenes. Simple pyrolysis of the fuel, which is presumably what is occurring to the secondary fuel, seems to prevent the formation of fullerenes. This pyrolysis may be preventing the breakdown of the fuel into radicals and molecules that are precursors to fullerenes. Without combustion to form these precursors, the secondary fuel has no mechanistic pathway for 
becoming fullerenes. Additionally, there may be some sort of barrier to fullerene formation that can only be overcome by combustion.

This scenario of a combustion process requirement is bolstered by the appearance that the secondary fuel is creating conditions very similar to the early stages of the McKinnon flame, prior to the primary flame reaction zone and to the onset of fullerene formation. Figure 4-11 through 4-13 show HPLC chromatograms of three extracted soot samples collected at various points along the McKinnon flame and two-stage flame \#3. Figure 4-11 shows the chromatogram from the extract of soot from the two-stage flame taken at $60 \mathrm{~mm} \mathrm{HAB}$, while Figure 4-12 is the McKinnon flame at $50 \mathrm{~mm} \mathrm{HAB}$ and Figure 4-13 is the McKinnon flame at 5.8 mm HAB.

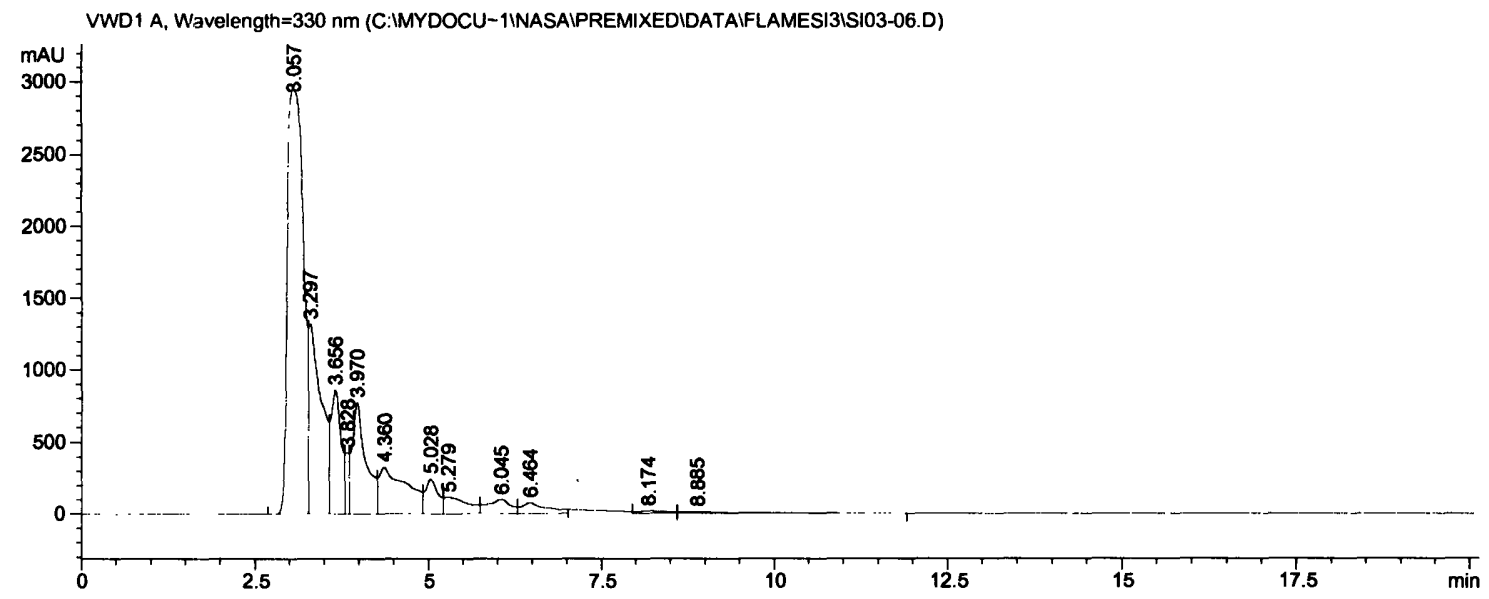

Figure 4-11. HPLC chromatogram of soot extract from two-stage flame \#3 at $60 \mathrm{~mm}$ $H A B$.

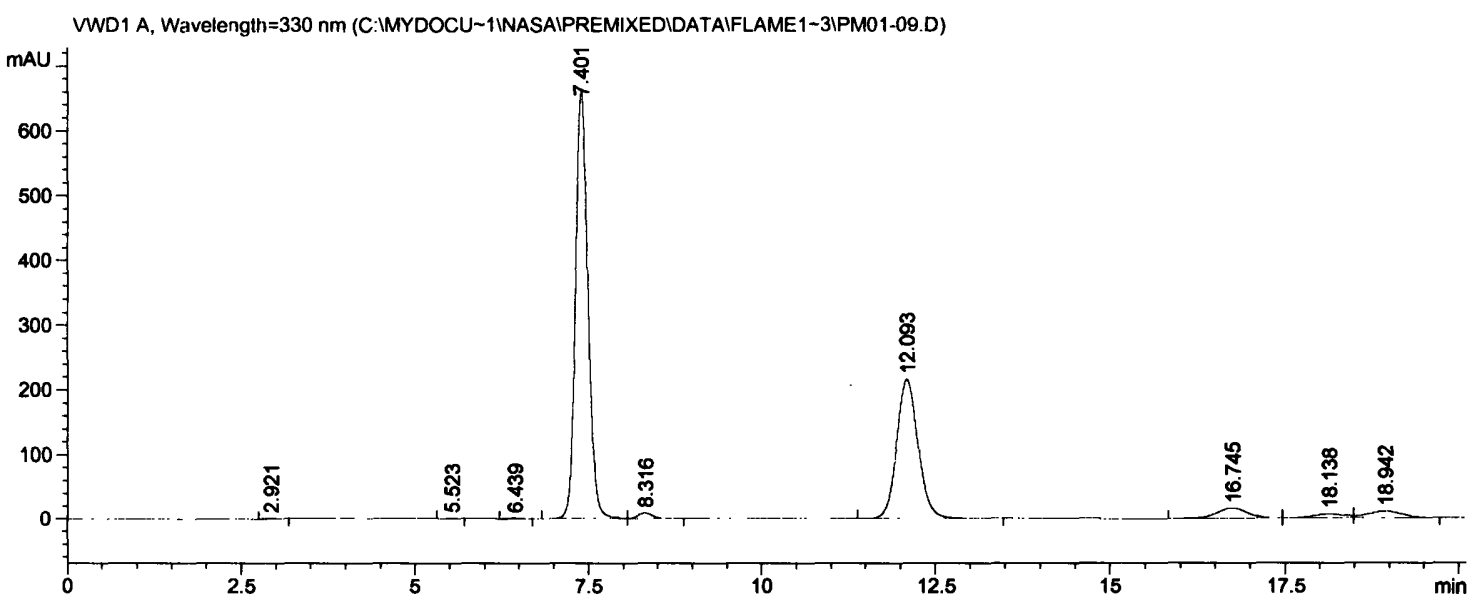

Figure 4-12. HPLC chromatogram of soot extract from McKinnon flame at $50 \mathrm{~mm} \mathrm{HAB}$. 


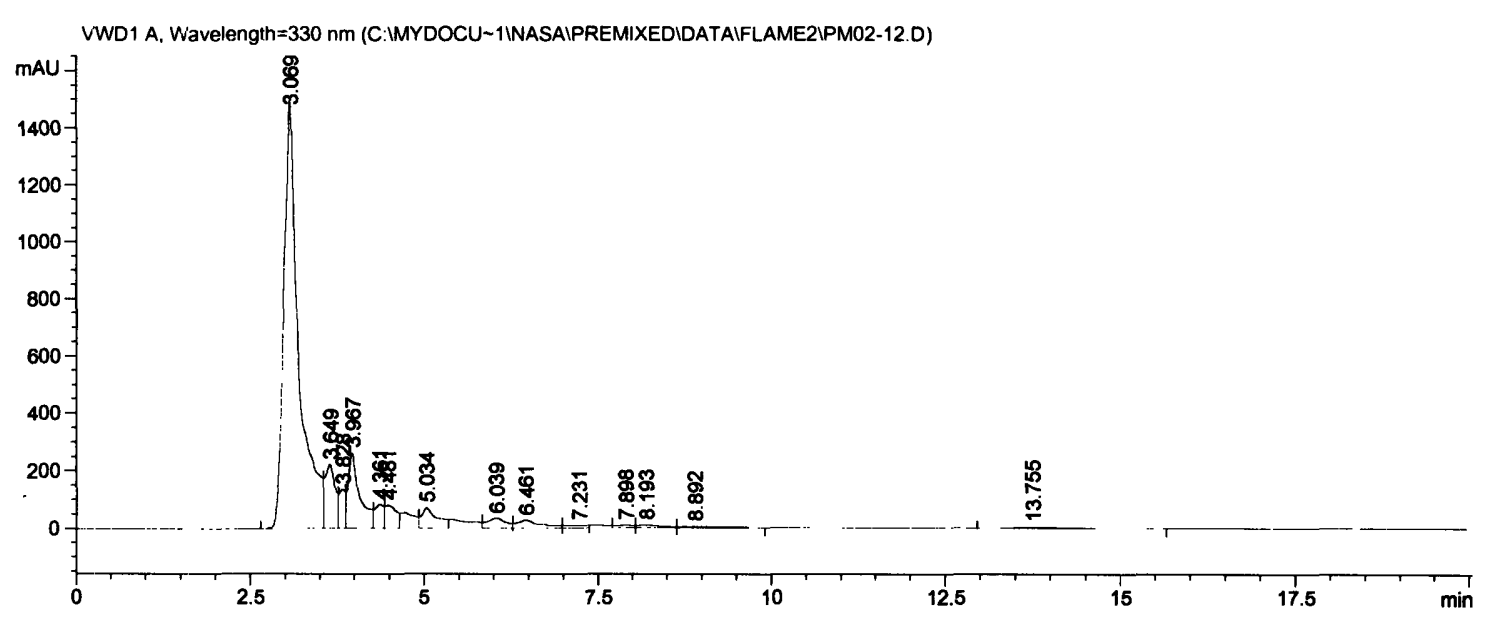

Figure 4-13. HPLC chromatogram of soot extract from McKinnon flame at $5.8 \mathrm{~mm} \mathrm{HAB}$.

Comparison of Figures 4-11 and 4-12 highlights the differences between the twostage flame and the McKinnon flame. Had secondary fuel been creating conditions similar to corresponding locations in the McKinnon flame, then these two chromatograms should have been roughly similar (accounting for the $15 \mathrm{~mm}$ HAB secondary fuel injection). However, it can be seen that they are starkly different. The two major peaks in Figure 4-12 at 7.5 and 12.0 minutes elution time correspond respectively to the presence of $\mathrm{C}_{60}$ and $\mathrm{C}_{70}$ molecules. No such peaks are seen in Figure 4-11. Had the absence of $\mathrm{C} 60$ concentration in the two-stage flame been simply a matter of magnitude, then the chromatogram should still have shown peaks, albeit much smaller ones, at 7.5 and $\mathbf{1 2 . 0}$ minutes. But in fact, the major peaks in Figure 4-11 all occur at less than 7.0 minutes indicating the presence of vast amounts of PAH. Since PAH are normally observed prior to fullerene formation [37], this indicates that the conditions even at later stages of the two-stage flame are similar to conditions in the early stages of the McKinnon flame.

This observation is strengthened by the comparison of Figure 4-11 with Figure 413. The chromatogram in Figure 4-13 shows the McKinnon flame at $5.8 \mathrm{~mm}$ HAB, which is prior to fullerene formation (Figure 4-5) and prior the primary reaction zone where combustion occurs. Figure 4-13 shows a prominence of peaks at less than 7.0 minutes, again indicating PAH like the peak in Figure 4-11 do. Beyond the mere appearance of peaks in the same region, these two figures show patterns of peaks that are remarkable similar. Both have their largest peaks at $\sim 3$ minutes with localized peaks at 
$\sim 3.65$ and $\sim 3.95$ minutes and a gradual tapering of the remaining signal. Such a strong similarity in peak patterns indicate the presence of similar concentrations of individual PAH species and thus similar flame conditions.

Such behavior and similarity may be expected as both chromatograms (Figures 411 and 4-13) come from regions of fuel pyrolysis. At such low HAB in the McKinnon flame, the fuel is being pyrloyzed prior to undergoing combustion slightly downstream. In the two-stage flame, the fuel is being pyrolyzed at much higher HAB as the oxygen has been mostly depleted when the fuel is injected and achieving pyrolysis is the whole goal of secondary fuel injection. As mentioned above, pyrolysis does not result in the formation of precursor molecules that may be necessary for fullerene formation. The combustion reactions necessary for these precursors occur in the McKinnon flame subsequent to $5.8 \mathrm{~mm} \mathrm{HAB}$ whereas no such combustion occurs higher than $60 \mathrm{~mm} \mathrm{HAB}$ in the two-stage flame. Additionally, combustion could possible have another effect as there may some barrier, energy or other, that prevents fullerene formation and can only be overcome by combustion. In any case, fullerene formation occurs in the McKinnon flame but not in flame \#3 even though their conditions are similar at the two selected points.

Based on these results, it seems that two-stage flames are not at all suitable for fullerene production. The data indicate that something is occurring in the combustion process that allows for fullerene formation, either through precursor generation or overcoming a barrier. The absence of fullerenes when fuel is injected into gases that are seemingly ideal for fullerene formation indicates that increasing fullerene yield in combustion requires a much more detailed understanding of the mechanisms involved with the production of fullerenes. This highlights the great need to understand what is occurring in a fullerene-forming flame and what reactions are involved in both the formation and consumption of fullerenes. This gap in understanding provides a major motivation for the fullerene reaction work outlined in the next chapter.

\subsubsection{Summary}

Six different two-stage flames were characterized for fullerene concentration and the data from all six indicate that secondary fuel has an adverse effect on the formation of 
fullerenes. In the two flames that had sooting primary flames, the concentration of fullerenes in the flame dropped immediately after the secondary fuel injection and never fully recovered to previous concentrations in the region studied. Those flames with a nonsooting primary flame, that is flames with no primary fullerene production, showed no fullerene production at all except for some trace amounts at long residence times. HPLC chromatograms for various samples indicated that the secondary fuel may be creating conditions that are very similar to the early stages of the McKinnon flame, a single-stage premixed flame known to be fullerene-forming. This similarity might be expected as both are regions of fuel pyrolysis. It is believed that such pyrolysis does not result in the precursors necessary for fullerene formation, which may be formed by combustion. Additionally, there may be some sort of barrier to forming fullerenes that can only be overcome by combustion. Consequently, it appears that the combustion process is necessary for making fullerenes even if the flame gases are otherwise ideal for fullerene formation. Two-stage flames seem to be unsuitable for the production of fullerenes. This highlights the need to better understand fullerene formation and consumption mechanisms before yield can be increased.

\subsection{Conclusions}

The present study of premixed flames extends and adds to the premixed studies performed previously by McKinnon and Grieco [37, 117]. Although there were some discrepancies between the current data and Grieco's data, the data trends and orders of magnitude were sufficiently similar to allow for further study with the confidence that the same flame was being investigated.

The McKinnon flame study was extended to include a characterization of several two-stage flames for fullerene concentration. The flames all used the McKinnon flame as a baseline but none of them were suitable for fullerene production and indicated that the secondary fuel was having an adverse effect on fullerene formation. Furthermore, HPLC chromatogram results indicate that the secondary fuel is creating pyrolysis conditions similar to those seen in the very early stages of the McKinnon flame where pyrolysis is also occurring. This supports the hypothesis that combustion is needed for fullerene production, either through precursor production or overcoming some sort of formation 
barrier. Because of the lack of promise in producing fullerenes, further and more detailed characterizations of two-stage flames were not performed.

The coexistence of soot and fullerenes in the McKinnon flame (when diffusion flame data indicated that fullerenes are consumed by soot) and the unexpected two-stage flame results emphasize the lack of understanding of fullerene formation and consumption reactions. Having a better understanding of such mechanisms would help in designing flames more promising for higher fullerene yields. Completing the objectives for premixed flames has provided the motivation and justification for investigating the fullerene mechanism in more detail by studying the reaction of fullerenes with soot. This is the focus of the next chapter. 


\section{Reaction rate coefficient of fullerene $\left(\mathrm{C}_{60}\right)$ consumption by soot}

The flame studies presented in the previous two chapters provide some evidence that can be interpreted to mean that fullerenes might be consumed by reaction with soot material in situ, but also provide some evidence that this is not the case. In diffusion flames, it was found that fullerene concentrations peak at the stoichiometric surface of the flame just prior to the onset of significant soot formation (Section 3.2.3.1). This observation can be interpreted to mean that fullerene molecules might be consumed by soot material. Similarly, in premixed flames, Grieco et al. [89] observed a severe drop-off in the flame concentration of fullerenes at relatively long residence times in the postflame zone of a sooting flame (Figure 4-6). The region of fullerene loss occurs within a region of continued soot production, suggesting that fullerenes might be consumed by soot. However, the present premixed study (Figure 4-5) along with Grieco's data at relatively short residence times suggest otherwise. They show that fullerenes continue to be formed even in the presence of soot, in apparent contradiction to the former theory.

Other studies have drawn conclusions about fullerene consumption by soot that appear to be conflicting or might be indicative of different behavior under different conditions. Mochida et al. $[119,120]$ found that fullerenes are carbonized at temperatures in the range of $473-2673 \mathrm{~K}$ by heat treatment of fullerene-containing soot produced by graphitic carbon vaporization in an electric discharge. However, Fuller et al. [121] and Burden et al. [97] observed the formation of fullerene molecules from the electron irradiation of soot also produced in an electric discharge.

To determine the behavior of fullerene molecules in the presence of combustiongenerated carbon particles pertinent to soot and to test the hypothesis that a consumption reaction occurs, fullerene molecules were exposed to commercially-produced carbon black, used as a well-characterized soot surrogate, at temperatures pertinent to the postflame zone of sooting flames. The resulting data sets were analyzed quantitatively to determine the rate coefficient of the observed fullerene-soot reaction. This rate coefficient was evaluated by comparing it to the theoretical upper limit from simple collision theory and it was used to help interpret previous evidence that fullerenes may be consumed by reaction with soot during the combustion synthesis of fullerenes. It was found that 
although fullerenes do react with soot in a manner that consumes them (consistent with first-order kinetics), the reaction rate is not enough to be significant when extrapolated to flame conditions, indicating that fullerenes are consumed primarily by other mechanisms.

\subsection{Experimental system and techniques}

The experiments consisted of subliming pure $\mathrm{C}_{60}$ fullerenes into a passing argon stream that was then passed through a soot-laden quartz wool bed. After passing through the bed, the gas contacted a water-cooled probe where any unreacted fullerenes condensed out. The reactor setup was designed to allow a continuous flow of gas over the heated sample and also to allow the collection of vaporized material downstream of the reaction bed.

\subsubsection{Reactor and furnace setup}

The setup for the heat-treatment experiments is shown in Figure 5-1. The reactor consists of a quartz tube ( $17 \mathrm{~mm} \mathrm{ID} \mathrm{x} 22 \mathrm{in}$.) which contains a combustion boat (Coors Model 60032) with pure $\mathrm{C}_{60}$ fullerenes upstream from a quartz-wool plug containing an approximately uniform distribution of carbon black. The quartz tube is positioned inside a $305 \mathrm{~mm}$ long x $50 \mathrm{~mm}$ ID tubular furnace (Lindberg Model 55036) whose edges are indicated in the figure.

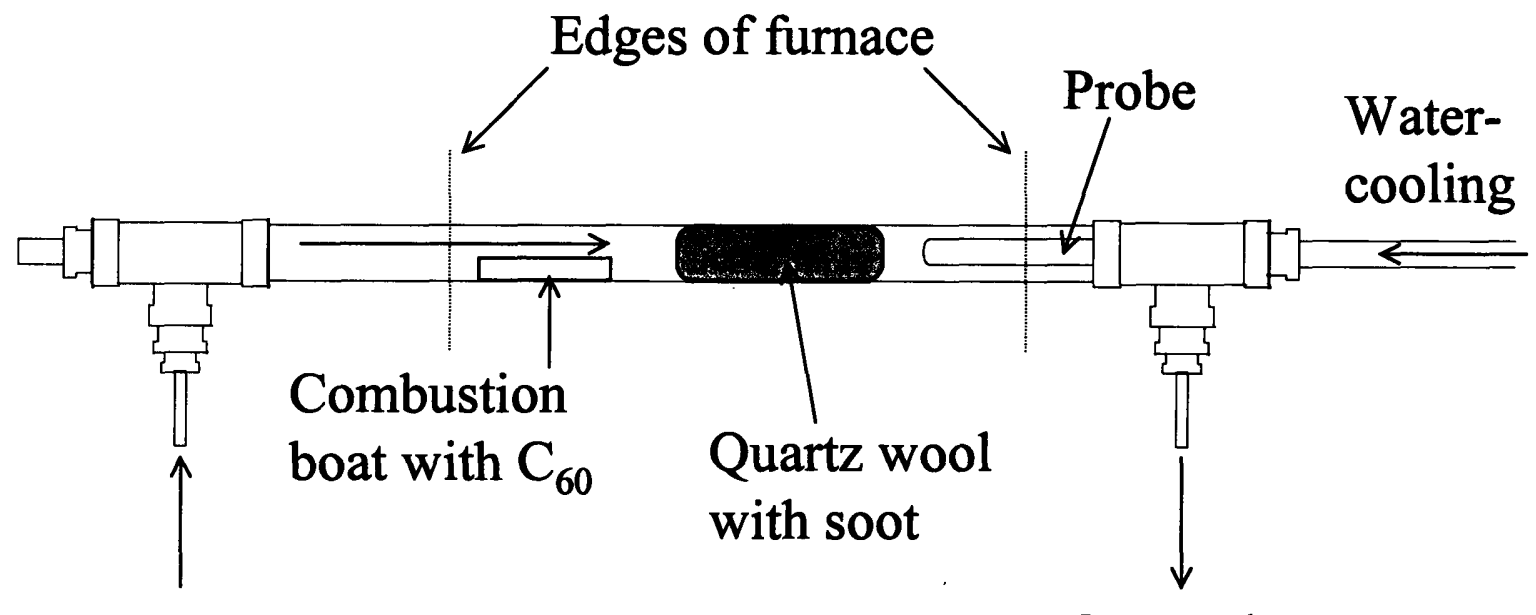

Gas inlet

Gas outlet

Figure 5-1. Schematic of equipment for study of fullerene-soot reaction 
The combustion boat was placed sufficiently upstream of the quartz wool to allow for radial diffusion of $\mathrm{C}_{60}$ prior to reaction. This arrangement ensured a uniform crosssectional profile of $\mathrm{C}_{60}$ concentration at the beginning of the reaction zone. The system temperature was monitored with a thermocouple located inside and midway along the furnace, between the quartz tube and the furnace tube. The residence time of fullerenes inside the reaction bed was calculated as the ratio of the bed length to the argon gas velocity.

\subsubsection{Vapor collection}

Argon gas flow is introduced at one end of the reactor while a water-cooled collection probe made of Pyrex $®$ glass (12 mm OD) is mounted at the other end upstream of the exhaust. As indicated by the arrows in Figure 5-1, the gas enters the reactor, passes over the combustion boat, and proceeds past the water-cooled probe, where condensable material is deposited. Gas flow to the reactor is controlled with a metering valve in conjunction with an upstream pressure regulator. The flow is measured and monitored with a mini-Buck Flow Calibrator (Model M-30).

Fullerenes sublime into the argon gas stream and are carried through the sootladen quartz wool. The fullerenes react with the soot along the length of the bed and any unreacted fullerenes physically condense, and are collected, on the water-cooled glass probe, whose temperature and surface area are designed to ensure the condensation of any vapor material.

\subsubsection{Analysis}

The initial mass of fullerenes used inside the combustion boat was measured gravimetrically; the post-reaction mass was determined by HPLC. Once the material is deposited on the probe, it can be collected at the conclusion of cool-down by submerging the probe in toluene and sonicating. These samples generally do not require extraction as species other than fullerenes are not observed. After collection, the toluene-fullerene samples are analyzed by HPLC in the same manner as described in Section 3.1.5. In these experiments, however, the total mass of fullerenes collected, not the concentration, was the primary result of interest. 


\subsubsection{Experimental conditions}

Prior to experimental runs, base case runs were performed with no quartz wool and with soot-free wool to determine the extent of $\mathrm{C}_{60}$ loss due to reactions other than those with the soot or due to filtration by the quartz wool. No such loss or reactions were observed. Additionally, it was observed that the sublimation of pure $\mathrm{C}_{60}$ resulted in a $20 \%$ residue of non-volatile material, consistent with previous studies [122]. The initial weight of the fullerenes in each subsequent run was corrected by this factor.

The fullerenes were $\mathbf{9 9 . 5 \%}$ pure $\mathrm{C}_{60}$ from Hoechst Corporation. Commercial carbon black was used as a surrogate for lab-generated soot because its physical properties are well-characterized and it is commercially-available for reproduction or extension of the experiments. The carbon black, Regal 330 from Cabot Corporation, had an average particle diameter of $33 \mathrm{~nm}$. The bulk density of carbon black in the plug of quartz wool in the reactor was $0.0083 \mathrm{~g} / \mathrm{cm}^{3}$, so the specific surface area of the carbon black in the bed, $S A_{\text {soot }}$, was $7551 \mathrm{~cm}^{2} / \mathrm{cm}^{3}$. The porosity of the quartz wool with deposited carbon black in the quartz tube was $98 \%$ and a new bed was used for every experimental run. An experiment time of nine minutes was used to ensure maximum $\mathrm{C}_{60}$ sublimation at all temperatures studied.

Experiments were run with a constant four-second residence time at different furnace temperatures in the range of 1023-1273 K. The reaction bed length was $6.1 \mathrm{~cm}$ and the argon flowrate for the difference experiments was in the range $50-60 \mathrm{~cm}^{3} / \mathrm{min}$ (at $273 \mathrm{~K}$ and $1 \mathrm{~atm})$.

\subsection{Reaction rate results}

It is assumed that the gas flow through the reaction cell is plug-flow, thus allowing the bed length to be correlated directly to residence time. To obtain a rate coefficient for the reaction of fullerenes with soot, the following first-order reaction rate expression was used:

$$
d\left[C_{60}\right] / d t=-k\left[C_{60}\right] S A_{\text {soot }}
$$


where $\left[C_{60}\right.$ ] is the concentration of $\mathrm{C}_{60}, t$ is time, $k$ is the rate coefficient, and $S A_{\text {soot }}$ is the soot surface area per unit volume. To simplify the expression, it is also assumed that the fullerene-soot reaction is heterogenous with $\mathrm{C}_{60}$ being the limiting reactant and soot surface area constant. This allows the incorporation of the soot surface area into the rate constant, giving

$$
d\left[C_{60}\right] / d t=-k^{\prime}\left[C_{60}\right]
$$

where $k^{\prime}=k S A_{\text {soot }}$. Integration of Equation 5-2 in terms of fullerene mass instead of concentration gives

$$
\ln \left[M\left(C_{60}\right) d M\left(C_{60}\right)_{d} d=k^{\prime} t\right.
$$

where $M\left(C_{60}\right)_{o}$ is the initial mass of fullerenes, $M\left(C_{60}\right)_{f}$ is the mass of unreacted fullerenes collected downstream from the reactor, and $t$ is the gas residence time in the reactor bed. Defining $k^{\prime}=A \exp \left(-E_{a} / R T\right)$ and taking the natural logarithm of both sides, the final rate expression is

$$
\ln \ln \left[M\left(C_{60}\right)_{d} M\left(C_{60}\right)_{d} d-\ln t=\ln A-E_{a} / R T\right.
$$

According to Equation 5-4, a plot of the left side of the equation against $1 / T$ should give a straight line with a slope of $-E_{a} / R$ and a y-intercept of $\ln A$.

A plot of the collected data is shown in Figure 5-2. It can be seen that the data fall into a linear trend, thus allowing the parameters of the reaction rate coefficient to be calculated from the graph. Using the quoted value of $S A_{\text {soot }}$ and accounting for the scatter of the data with error limits of one standard deviation, the following expression for $k$ is obtained:

$$
k=0.0042( \pm 0.0002) \exp [-4914( \pm 580) / T] \mathrm{cm} \mathrm{s}^{-1}
$$


The data in these experiments were collected over two different days and the daily data sets are represented individually in Figure 5-2. It can be seen that data collected on the same day are perfectly collinear but the slopes of lines are somewhat different between the two days' data. The reason for this difference is unknown. Thus the two sets of data yield similar but not exactly the same kinetic rate coefficients. The parameters given in Figure 5-2 are obtained from a regression of all the data together.

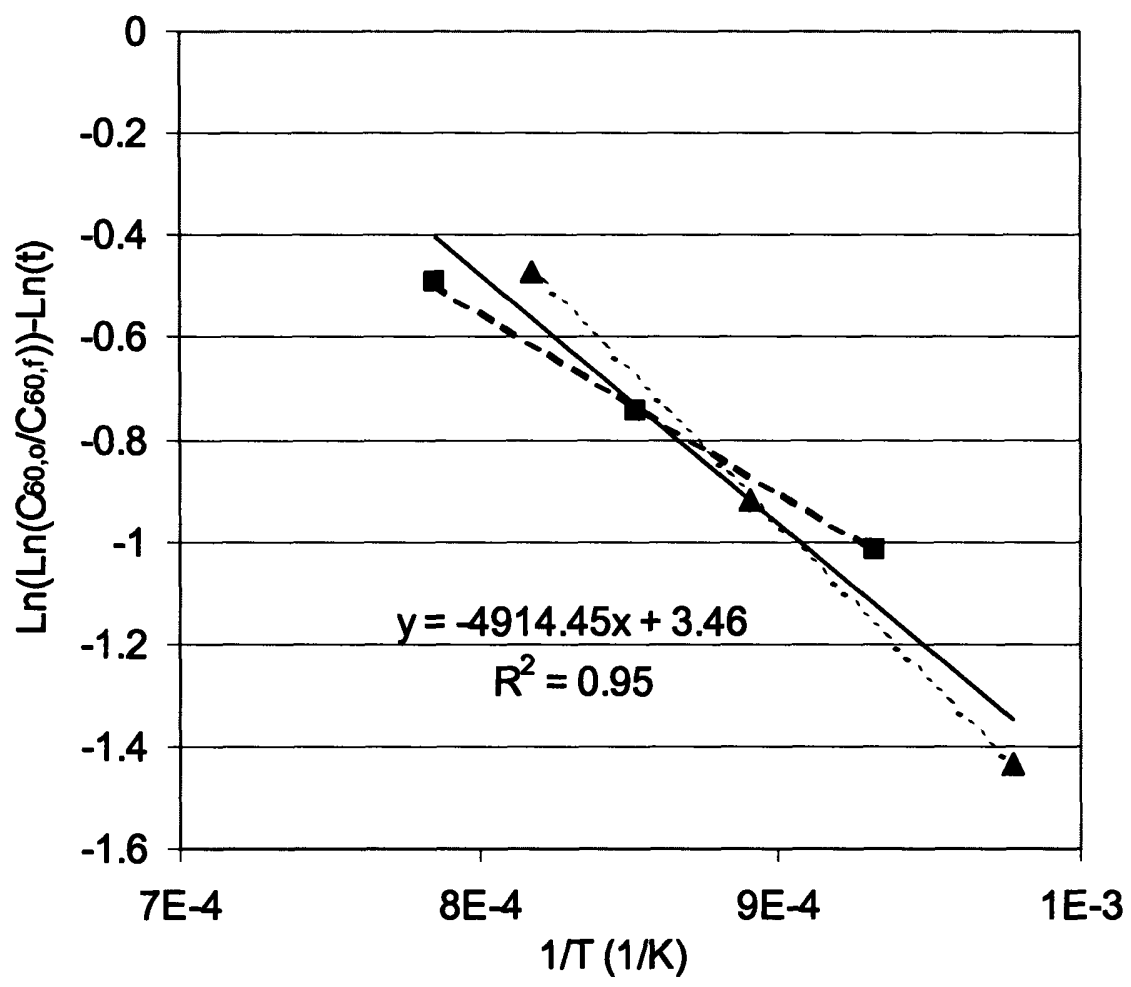

Figure 5-2. Fullerene-soot reaction rate plot - Day one data (๓) with regression (longdashed line), Day two data $(\mathbf{\Delta})$ with regression (short-dashed line), and full regression (solid line) with equation.

\subsection{Discussion}

Consistent with the diffusion flame study (Chapter 3 ) and the latter portion of Grieco's premixed flame study, the results from the plot shown in Figure 5-2 indicate that fullerenes are indeed being consumed by soot and that the reaction is dependent on temperature in a manner that is consistent with first-order kinetics. It might be possible that the decline in fullerene mass is due in part to fragmentation of the fullerene molecules in addition to consumption by soot. However, previous studies have shown that $\mathrm{C}_{60}$ is thermally stable in inert gas up to $1720 \mathrm{~K}[123,124]$. As the temperature in 
these experiments did not exceed $1300 \mathrm{~K}$, it is unlikely that any unimolecular fragmentation occurred. Similarly, Whetten and Yeretzian found that fragmentation of fullerenes due to surface collision, independent of the surface or temperature, required an unusually high impact energy [125]. This makes it unlikely that any collisional fragmentation of the fullerene molecules occurred. The exclusion of these two mechanisms leaves reaction with soot as the apparent mechanism for observed fullerene consumption.

To determine whether the fullerene-soot reaction observed in this experiment could explain the rates of fullerenes consumption previously observed in the McKinnon flame and tentatively attributed to a possible fullerene-soot reaction, and also to see if consumption is significant in the present premixed study (Section 4.2), the present data were used in the following analysis. From simple collision theory, the collision rate of $\mathrm{C}_{60}$ with the surface of soot particles is:

$$
k_{\text {coll }}=\left[k_{B} T /(2 \pi m)\right]^{1 / 2}\left[C_{60}\right]
$$

where $k_{B}$ is Boltzmann's constant and $m$ is the mass of one molecule of $\mathrm{C}_{60}$. If $T$ is expressed in degrees $\mathrm{K}$ and $\left[C_{60}\right]$ in $\mathrm{mol} / \mathrm{cm}^{3}$, Equation 5-6 can be expressed as

$$
k_{\text {coll }}=135.5 T^{1 / 2}\left[C_{60}\right] \mathrm{cm} \mathrm{s}^{-1}
$$

Using the collision rate as a basis for expressing the reaction rate and defining $\gamma$ as the fraction of the collisions resulting in reactions, or collision efficiency, the reaction rate can be written

$$
d\left[C_{60}\right] / d t=-k_{\text {coll }} / S A_{\text {soot }}
$$

Eliminating $k_{\text {coll }}$ between Equations 5-7 and 5-8, we have

$$
d\left[C_{60}\right] / d t=-135.5 T^{1 / 2}\left[C_{60}\right] \gamma S A_{\text {soot }}
$$


Comparison of Equations 5-1 and 5-9 reveals that

$$
k=135.5 T^{1 / 2} \gamma
$$

Eliminating $k$ between Equation 5-5 (without the uncertainties) and Equation 5-10 and solving for $\gamma$ :

$$
\gamma=3.1 \times 10^{-5} T^{-1 / 2} \exp [-4914 / T]
$$

Collision efficiencies computed from Equation 5-11 are less than unity as they should be and they rise with temperature qualitatively as might be expected, but the magnitude is very small at any temperature of interest. The efficiency is only $2 \times 10^{-8}$ at the upper temperature limit of this experiment, i.e. $1273 \mathrm{~K}$. This rate would be insignificant on the time scale of flame reactions. If the observed temperature dependence remained valid up to flame temperature, the indicated collision efficiency would still be insignificant at flame temperatures, e.g., $\gamma=6 \times 10^{-8}$ at $2000 \mathrm{~K}$, as described below.

The measured collision efficiency was applied to fullerenes data from Grieco's premixed flame study and the present premixed study of the McKinnon flame. Soot particle size data obtained by McKinnon [117] were utilized in conjunction with fullerene concentration, temperature, and soot mass concentration data from Grieco et al. [89] and from Chapter 4 (Figures 4-5 and 4-6) to see if the measured reaction rate could explain the rate of fullerenes consumption corresponding to the observed decline in fullerene concentration in post-flame zone (Figure 4-7) and to see if the reaction is significant during the observed increases in fullerene concentration (Figure 4-5). Of course, Grieco's soot concentration data was used for calculating his fullerene consumption rates while the present soot data was used for consumption rates in the present flame. Although the soot particle size data only extend $50 \mathrm{~mm}$ above the burner while the drop-off in fullerenes concentration is observed in the region of approximately $70-80 \mathrm{~mm}$, size information in the latter region was obtained by extrapolation. Soot surface area per unit volume of flame gases at different distances from the burner was computed from the profiles of soot mass concentration and particle size, taking into account approximately the reduction in 
surface area due to particle agglomeration using electron micrscope images of the soot [99].

The consumption rates of $\mathrm{C}_{60}$ at different heights above the burner were computed using Equations 5-9 and 5-11 and the experimental temperature profile [117]. The results for Grieco's McKinnon flame data are shown in Figure 5-3 and those for the present McKinnon flame data are shown in Figure 5-4. It is seen that the data in both of these figures follow the general trends of the respective data on which they are based. The decline in consumption above $80 \mathrm{~mm} \mathrm{HAB}$ in Figure 5-4 is primarily due to the lower temperatures observed later in the flame. The differences in the consumption rates are also reflective of the relative differences between the two fullerene and soot concentration data sets (Figures 4-5 to 4-8). However, the overall magnitude of both consumption rate data sets is still $\sim 10^{-17}$ or $10^{-16}$.

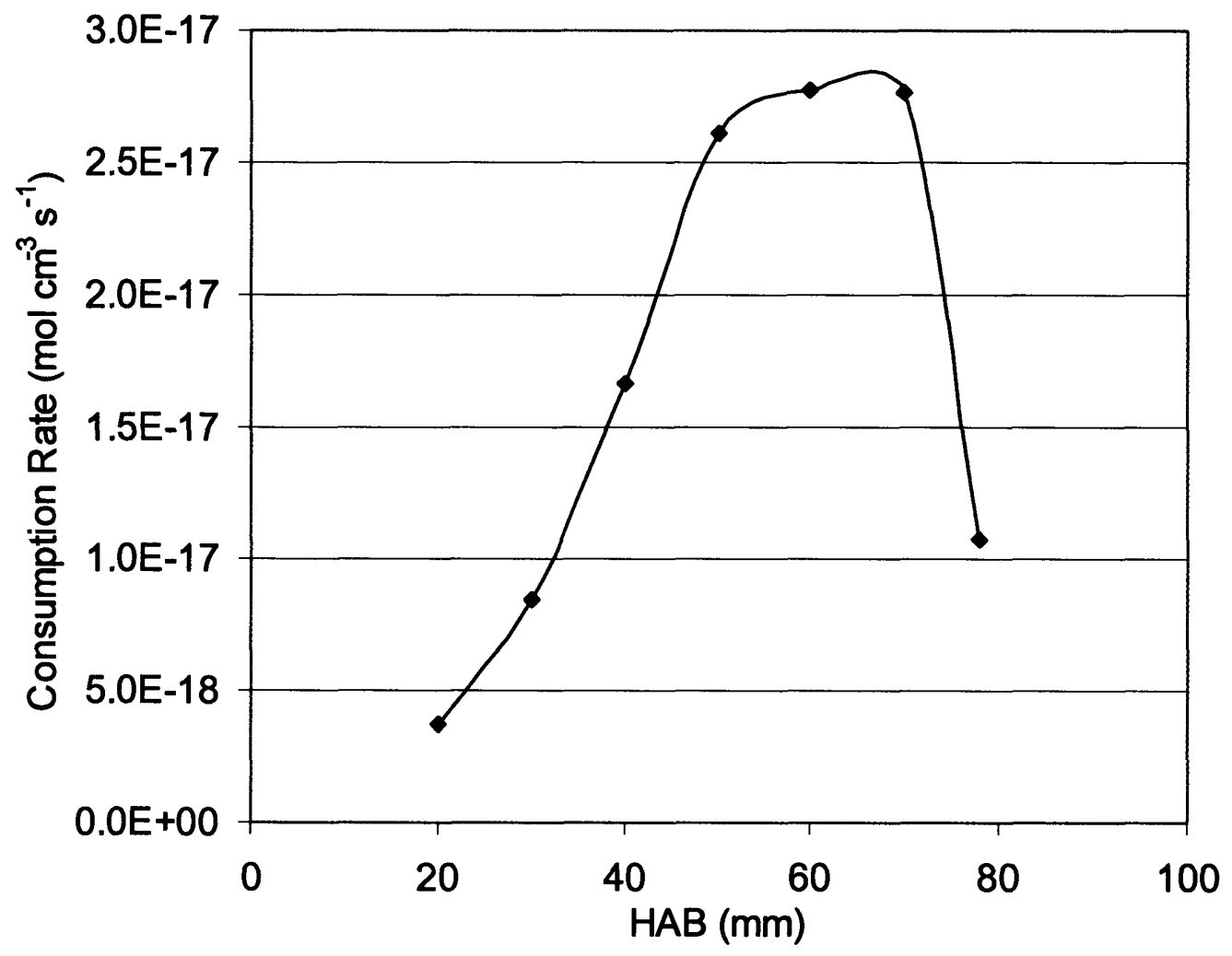

Figure 5-3. Predicted consumption rate of fullerenes by soot in the McKinnon flame using fullerene and soot data from Grieco's study [89]. 
Although the validity of Equation 5-11 and hence the accuracy of the calculation are unknown at temperatures above $1273 \mathrm{~K}$ and the flame temperature varies from about $2000 \mathrm{~K}$ to about $1350 \mathrm{~K}$ over the region $20-80 \mathrm{~mm}$ above the burner where the main fullerenes formation and consumption occur (Figures 4-5 and 4-6), the results are nevertheless of interest for primary analysis. In Grieco's data (Figure 4-6), in the region of the fullerene concentration drop-off at 70-80 $\mathrm{mm}$ above the burner, the net rate of fullerene $\mathrm{C}_{60}$ consumption calculated from the data is about $2 \times 10^{-10} \mathrm{~mol} \mathrm{~cm}^{-3} \mathrm{~s}^{-1}$ which is a factor of $10^{7}$ larger than the rate calculated for that region of the flame based on the data of this study (Figure 5-3).

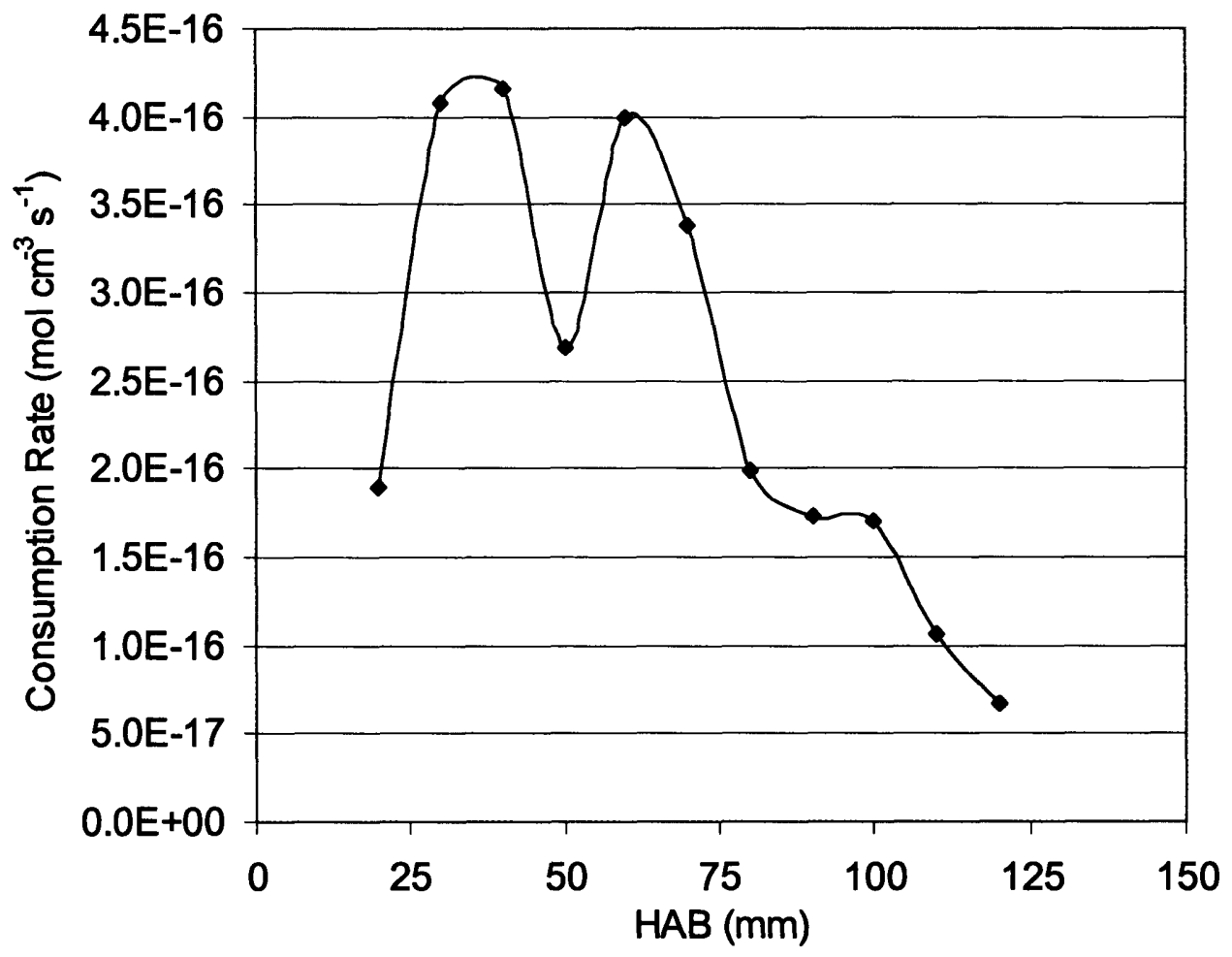

Figure 5-4. Predicted consumption rate of fullerenes by soot in the McKinnon flame using fullerene data from the present study.

Evidence for fullerenes consumption can also be seen earlier in the flame where fullerenes concentration is increasing in the region $20-70 \mathrm{~mm}$ above the burner, as well as in the entire range of data in Figure 5-4. Although the net rate of fullerenes reaction in these regions is clearly formation, it can be assumed that consumption reaction such as that evidenced at $70-80 \mathrm{~mm}$ in Figure 5-3 is also occurring in the other regions where they compete with, and are overshadowed by, formation reactions. As shown by 
Kronholm et al. [94] in the case of soot formation, analysis of the competition between formation and destruction can account for oscillations in the concentration profiles such as those seen for fullerenes in Figure 4-5 and 4-7. Accordingly, the net rate of fullerene formation is a relatively small difference between two large rates, i.e., the sum of all consumption reactions and the sum of all formation reactions. Subtle changes in either of these summed rates can result in significant changes in the net fullerenes reaction rate and account for the observed concentration oscillations. It can be calculated from the $\mathrm{C}_{60}$ concentration data in Figures 4-5 and 4-7 that the increase in $\mathrm{C}_{60}$ consumption rate that would be required to explain the decreased net rate of formation between about $40 \mathrm{~mm}$ and about $60 \mathrm{~mm}$ above the burner, and hence to account for the oscillation in the $\mathrm{C}_{60}$ concentration profile in that region, is approximately six orders of magnitude larger than the consumption rate estimated for that region based on the present data (Figures 5-3 and 5-4).

Thus the fullerenes-soot reaction studied in this experiment is much too slow and its observed temperature dependence is much too small to allow the rates predicted for the flame to be of any significance in the region of major net fullerenes consumption or the earlier region of smaller changes in the net rates of fullerenes reaction. If a fullerenessoot reaction were to be responsible for the fullerenes consumption rates in the flames, it would have to be a mechanism whose rate increases by a factor of $10^{6}$ or more going from the conditions of the experiment to flame conditions. For example, calculations show that temperature dependence of the mechanism would have to be equivalent to an overall activation energy of about $160 \mathrm{kcal} / \mathrm{mol}$ to increase the rate of the fullerenes-soot reaction observed here to the incremental $\mathrm{C}_{60}$ consumption rate possibly reflected by the above-mentioned oscillation in the $\mathrm{C}_{60}$ concentration profile in the region $40-53 \mathrm{~mm}$ from the burner (Figures 4-5 and 4-7) where the temperature is about $1700 \mathrm{~K}$. Similar calculations for the 70-80 mm region where $\mathrm{C}_{60}$ concentration drops (Figure 4-7) and the temperature is about $1380 \mathrm{~K}$ show that the required overall temperature dependence would have to be the equivalent of a $490 \mathrm{kcal} / \mathrm{mol}$ activation energy to increase the fullerenes consumption rates observed in this experiment to that seen in the flame. The latter value is so large as to suggest that the mechanism of the fullerene-soot reaction 
studied in the present experiment would not play a significant role in the fullerenes consumption observed in the region of the large decrease of concentration.

The mechanism of fullerenes consumption by soot under the conditions of the present experiment has not been established. The magnitude of the observed activation energy, i.e., $E_{a}=9.8 \mathrm{kcal} / \mathrm{mol}$, would not be inconsistent with surface diffusion. Such a process might involve a reversible chemisorption of fullerene molecules on the soot with simultaneous surface diffusion to active sites where reactions with the soot material occurs. Given that the observed reaction rate is a small fraction of the collision rate, e.g., $y=2 \times 10^{-8}$ at $1273 \mathrm{~K}$, most of the fullerenes arriving at the soot surface would be either reflected or quickly desorbed according to this mechanism. An alternative explanation of the data might be a heterogeneous reaction between fullerenes and soot. In this interpretation, the observed activation energy would be the energy barrier for chemisorption, and this value along with the small magnitude of the observed collision efficiency would imply that a substantial fraction of the collisions have sufficient energy for reaction but that the probability of colliding at a suitable site in the soot structure is exceedingly low. In either mechanism, one might expect the population of radical centers or active sites in the soot structure to be important, and the role of reactions between the soot and flame radicals could be important. Accordingly, one might expect the temperature dependence of the concentration of radicals, which can be the equivalent of overall activation energies of around $50-70 \mathrm{kcal} / \mathrm{mol}$ in fuel-rich flames of interest in fullerenes formation, to give rise to a higher temperature sensitivity than was observed in this work. Therefore, if the mechanisms being considered were to be important, the overall activation energy of the fullerenes-soot reaction would be expected to exceed substantially the value observed here. The possibility of such higher activation energies and possibly a higher reactivity of in situ flame soot as compared to the collected carbon black used here might leave open the possibility of a significant role for the fullerenessoot reaction in flames. Another possibility for explaining the observed consumption of fullerenes in flames is the reaction of fullerenes with other combustion intermediates and products such as PAHs, carbon monoxide, flame radicals, etc. Investigations of these different possibilities are interesting topics for further research. 


\subsection{Conclusions}

Fullerene $\mathrm{C}_{60}$ reacts with soot represented by a commercially available carbon black in the temperature range 1023-1273 $\mathrm{K}$ with the fullerenes being consumed in accordance with first-order kinetics. The collision efficiency of the reaction is very small, of the order $10^{-8}$ at the temperatures studied, and the activation energy is $9.8 \mathrm{kcal} / \mathrm{mol}$. These characteristics would be consistent with reaction rate control by a surface diffusion controlled process or a heterogeneous reaction. Extrapolation of the observed consumption rates to flame temperatures using the observed activation energy would give a rate too small by a factor of $10^{6}$ to $10^{7}$ to explain the rate of fullerenes consumption observed in a fullerene-producing flame. The temperature dependence that would be required to allow the observed fullerene-soot reaction when extrapolated to flame temperatures to be significant compared to observed rates in flames is so strong as to suggest that mechanisms other than those studied here must control fullerenes consumption in flames. Such other mechanisms are reactions of fullerenes with other flame species and heterogeneous soot-fullerenes reactions with the reactivity of the soot dependent on soot reactions with other flame species, especially radicals. 


\section{Observations of fullerenes tethered to carbon black particles}

In the HRTEM images that have been analyzed thus far in support of the work done in this thesis, various observed carbon structures have been assumed to be fullerene molecules. For example, the circular structures seen in the inset of Figure 3-14 and in Figure 3-15 (particularly 3-15 (b)) were all believed to have been actual fullerene molecules. However, it is not yet certain that these structures are in fact fullerenes. Furthermore, up until this point, it has not been proven that fullerenes can definitively be seen by HRTEM analysis.

There is actually some debate currently in the literature as to whether circular structures seen in HRTEM are fullerenes or not. Several previous studies $[121,126]$ show HRTEM images of circular structures and treat them as though they were fullerene molecules. In contrast, Ajayan et al. [127] argue that a similar structure must be a singlewalled nanotube aligned perpendicular to the plane of the image because its contrast is similar to that of a polygonized carbon tube structure. Burden et al. [97] counter that such structures must be isolated fullerenes, rather than nanotubes, because nanotubes are unlikely to form in the conditions and manner described. Although it's quite possible that all the authors are correct in their assessments drawing on the differences in their respective experimental conditions, it still does not conclusively answer the question of whether fullerenes can or cannot be observed by HRTEM.

To answer this question, the present study was performed where pure fullerene molecules were chemically bound, or tethered, to the surface of carbon black particles and then analyzed quantitatively by HRTEM. Comparison of these analyses with an analogous analysis of pure carbon black indicates that fullerenes are successfully being tethered to the carbon black and furthermore, that observed circular carbon structures are indeed fullerenes proving definitively that they can be observed under HRTEM.

\subsection{Experimental}

Three different samples were prepared for investigation by HRTEM. The first sample consisted of pure carbon black suspended in a toluene solution. The carbon black used here is commercially available as Regal 330 carbon black from the Cabot Corporation. 
A second carbon black-toluene solution was prepared to which a specified amount of functionalized fullerene molecules were added. The functionalized fullerenes, obtained from the University of Kentucky [128], consisted of dichloromethano[60]fullerene. The properties of this compound and similar ones, such as dibromomethano[60]fullerene, have been studied extensively [128]. The functional group bridges two carbon atoms on the fullerene molecule and the halides can be readily substituted to provide a chemical tether to the compound of interest.

After a uniform dispersion was ensured with vigorous mixing, the toluene was allowed to evaporate and the resulting dry powder mixture was sealed inside an argonfilled glass tube. The entire unit was then placed inside a tubular furnace (Lindberg Model 55036) and heat treated at approximately $400^{\circ} \mathrm{C}$ for $4 \frac{1}{2}$ hours.

After sufficient cooling, the mixture was sonicated into toluene for 13 minutes to dissolve any untethered fullerenes. The sample was then extracted through a $0.45-\mu \mathrm{m}$ nylon filter under vacuum. Prior to extraction, however, a fraction of the sample was drawn-off to be analyzed as a pre-extraction sample. The remaining extracted solid was analyzed as the post-extraction sample.

All three samples were deposited onto lacey carbon grid supports and the toluene was allowed to evaporate in each case. The samples were then analyzed in a JEOL 2010 electron microscope operating at $200 \mathrm{kV}$.

The images obtained from the microscope were qualitatively analyzed for the presence of fullerene-type structures, or structures that appear fullerenic, meaning completely closed cages. Only the perimeters of photographed particles were analyzed as the internal carbon density is too great to allow for accurate structural characteristic assessment when a fullerene has a supporting particle below it [99]. In each image, the number of fullerene-type structures per length of perimeter was counted and the diameter of each of those structures was measured. The data was then aggregated across all the images of a particular sample to provide linear fullerene concentration data and fullerene size distribution data. 


\subsection{Tethering results}

Figures 6-1 and 6-2 show two images that are representative of the images analyzed from the different samples. Figure 6-1 is an image of a particle taken from pure carbon black while Figure 6-2 shows a particle from the post-extraction sample.

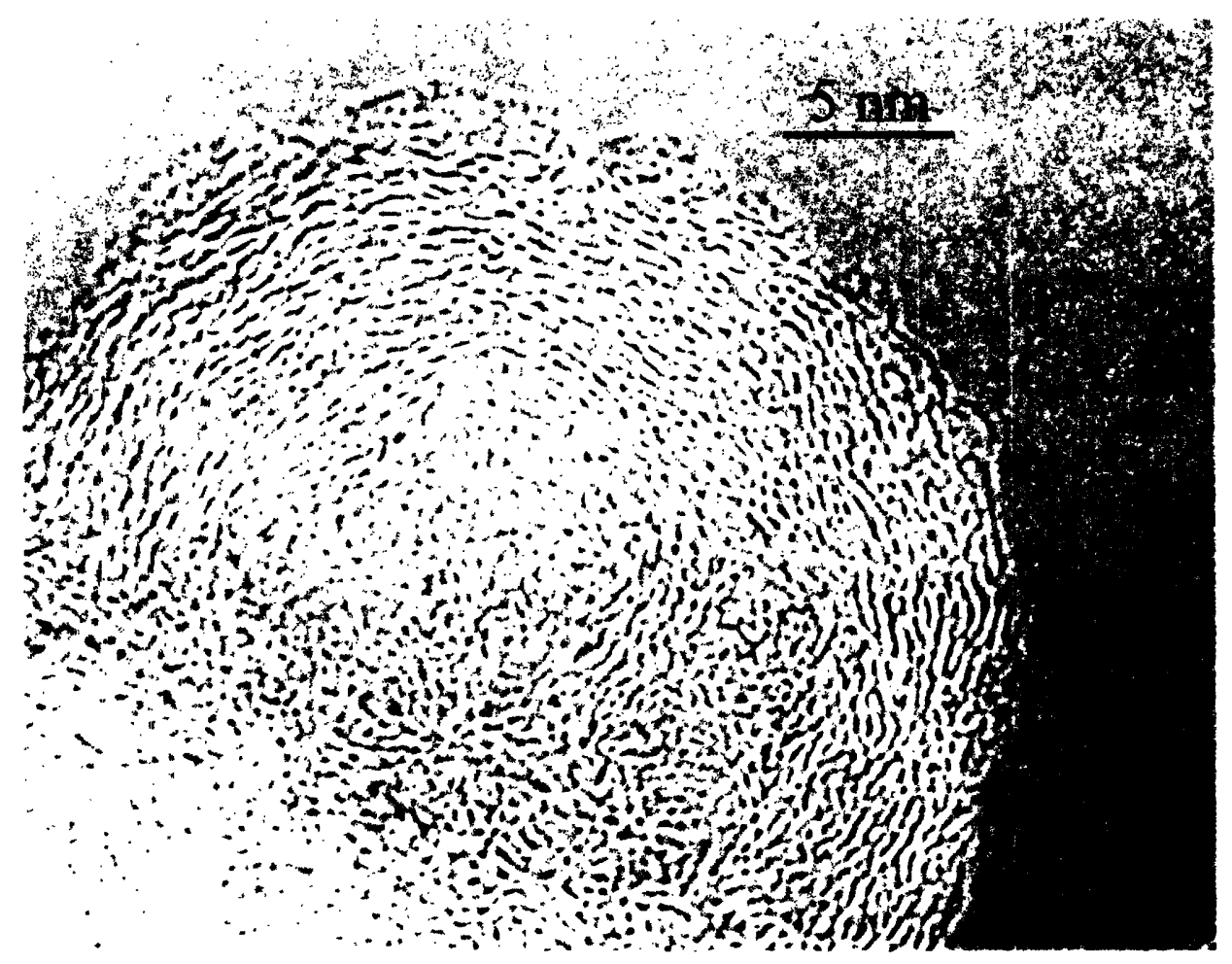

Figure 6-1. HRTEM images of particle from pure carbon black sample.

The black dashes in Figure 6-2 are user-added indications of structures that were deemed to be fullerenic and included in the concentration and size data. The absence of black dashes in Figure 6-1 indicates the lack of fullerene-type structures in the carbon black sample. Qualitatively, the images show quite clearly that the carbon black doped with tethered fullerenes has many more fullerene-type structures than the pure carbon black particles.

Quantitative analyses of the same images reinforces the qualitative observation. Table 6-1 gives a summary of the fullerene concentration data; Figure 6-3 shows the fullerene size distribution of the observed structures. 


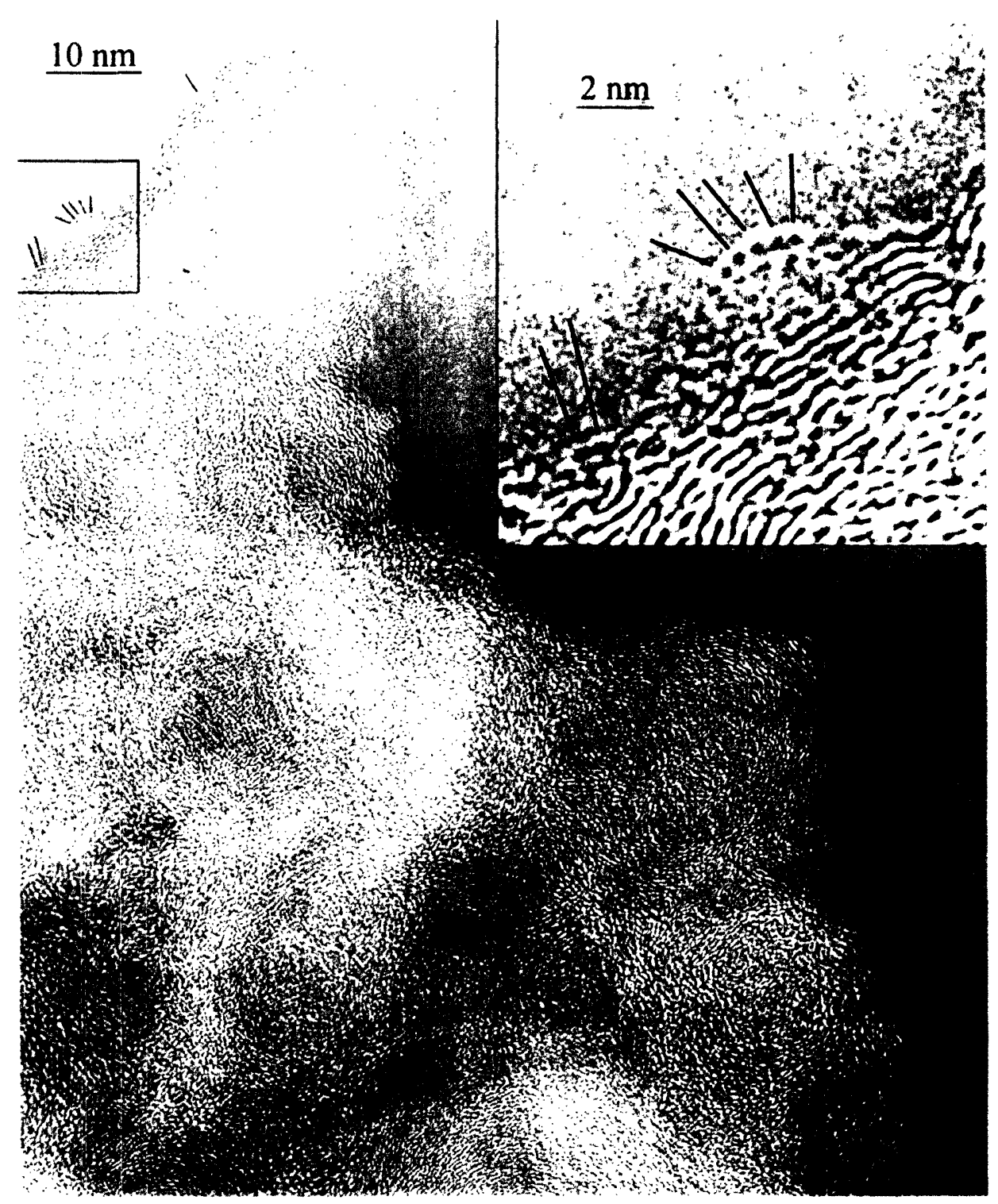

Figure 6-2. HRTEM images of particle from post-extraction tethered fullerene sample with user-entered dashes to identify fullerene-type structures.

Table 6-1. Fullerene-type structure linear concentration data.

\begin{tabular}{|l|c|c|c|}
\hline \multicolumn{1}{|c|}{ Sample } & $\begin{array}{c}\text { Fullerenic } \\
\text { Structures }\end{array}$ & $\begin{array}{c}\text { Perimeter } \\
\text { Length }(\mathrm{nm})\end{array}$ & $\begin{array}{c}\text { Fullerenic structures/ } \\
\text { perimeter length }\left(\mathrm{nm}^{-1}\right)\end{array}$ \\
\hline Carbon Black & 21 & 1775 & 0.012 \\
\hline Tethered $\mathrm{C}_{60}-$ Pre-extraction & 209 & 2220 & 0.094 \\
\hline Tethered $\mathrm{C}_{60}-$ Post-extraction & 172 & 1970 & 0.087 \\
\hline
\end{tabular}




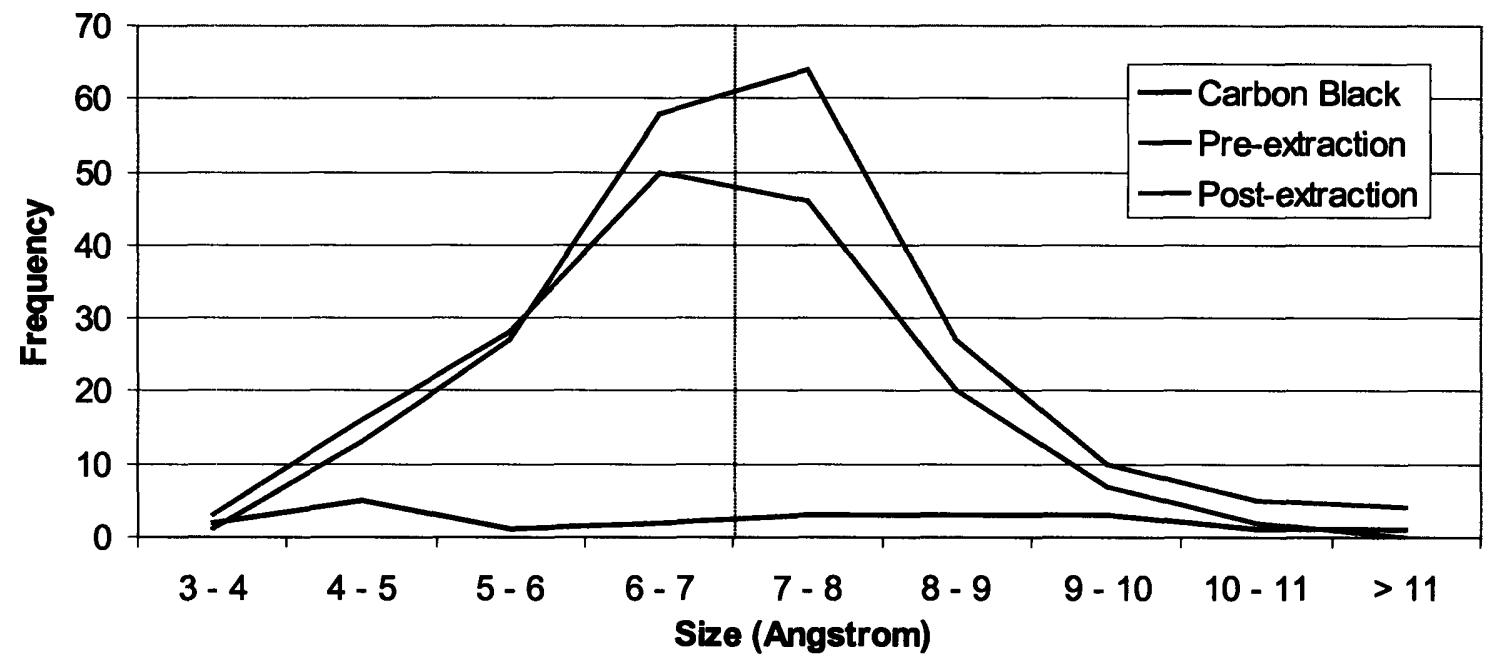

Figure 6-3. Fullerene-type structure diameter distribution data on an absolute basis.

From Table 6-1, it is seen that both samples containing tethered fullerenes have a fullerene concentration almost an order of magnitude greater than the pure carbon black sample. It should be noted that the post-extraction sample does have a slightly lower concentration than the pre-extraction samples. This is not surprising as it is expected that less than $100 \%$ of the functionalized fullerenes would react with the carbon black, leaving some untethered fullerenes to be separated during extraction.

It should also be noted that both the pre- and post- extraction samples exhibit concentrations less than what would be expected from theoretical calculations. From the relative amounts of carbon black and functionalized fullerenes utilized, and assuming a uniform surface distribution, it is expected that the area concentration of fullerenes would be 0.25 fullerenes $/ \mathrm{nm}^{2}$, translating to a linear density of 0.50 fullerenes $/ \mathrm{nm}$. Both tethered samples yield a density approximately $20 \%$ of this expected value indicating that many of the fullerenes are not observed. Possible explanations for this are discussed in the following section.

The size distributions in Figure 6-3 show that while the carbon black sample has no significant peaks, the tethered fullerene samples show strong peaks at $7 \AA$, which is the diameter of a $\mathrm{C}_{60}$ molecule and represented in the figure by the dotted line. Figure 6-3 
also shows that the carbon black distribution is, in total, significantly lower than the other two samples.

The size distributions indicate that most of the differences in concentration data between the carbon black and fullerene-doped samples are due to the addition of actual fullerene molecules.

\subsection{Discussion}

Previous researchers [126] have observed $\mathrm{C}_{60}$ in magnesium oxide in an experimental situation similar to that used in the present work. Given this precedent and the data presented above, we believe that the contrast observed in Figure 6-2, for instance, is consistent with single $\mathrm{C}_{60}$ molecules. The order of magnitude increase in observed fullerenic-type structures is strengthened by the emergence of a significant size distribution peak at $7 \AA$ in the tethered fullerene samples compared to the pure carbon black sample. This coupled with the qualitative observations indicates quite strongly that fullerenes have indeed been tethered to the carbon black surface, and furthermore, that these fullerenes are observable with HRTEM.

Nonetheless, precautions must be taken to reduce the influence of radiation damage and/or beam heating on the observations. Such influences include degradation of the sample [129], incorporation of smaller structures into larger ones, and migration of molecules. All three of these scenarios have been observed during HRTEM imaging and all can contribute to an artificially low frequency of fullerene observations. For example, we have observed $\mathrm{C}_{60}$, and other fullerenic molecules, migrating "behind" the carbon black under some observation conditions, in accord with earlier reports [121].

Precautions must also be taken to analyze images taken of a particular soot area at all possible focal lengths. The position of the soot particles along the optic axis of the instrument will create variations in contrast and image characteristics which can render some structures uninterpretable. This effect can compound errors due to other influences discussed above and again, can suppress successful observation of fullerenic structures. Through focal series can help to alleviate this source of error.

While care is taken to minimize the effects of these imaging artifacts, some error will still be incorporated into the imaging results. This reduction in fullerenic structure 
observations gives a plausible explanation as to why, as mentioned in the previous section, the observations account for only $20 \%$ of the expected theoretical value. Normally, a 20\% agreement would be cause for concern but given the fact that we still observe an order-of-magnitude increase in fullerenic structures with tethered fullerenes, our conclusions are not weakened.

\subsection{Conclusions}

Pure fullerene $\mathrm{C}_{60}$ molecules were functionalized with a dichloromethano group and this functional group was used to tether the $\mathrm{C}_{60}$ molecules to the surface of commercially available carbon black particles. Two different samples of the tethered compound were taken, one prior to toluene extraction and one subsequent, and both were analyzed by HRTEM for the abundance of fullerene-type structures and the size distribution of the diameters of those structures. The results were compared to similar observations of a pure carbon black sample and it was found that both tethered fullerene samples had approximately an order-of-magnitude greater occurrence of fullerene-type structures. Furthermore, the size distributions of the tethered samples both show a strong peak at $7 \AA$, the diameter of $\mathrm{C}_{60}$, while that of the pure carbon black shows no such peak. This indicates that the functionalized fullerenes have, in fact, tethered themselves to the carbon black particles and, more importantly, that these fullerenes are observable under HRTEM. The linear density of fullerenes in the tethered fullerene samples are about $20 \%$ of the expected value from experimental calculations, raising some concern. However, the well-documented imprecision and artifacts of HRTEM imaging, combined with the observed order-of-magnitude differences, preclude any weakening of the stated conclusions. 


\section{Economic cost modeling of commercial fullerenic soot plant}

The overall goal for combustion synthesis research is to provide an alternative to graphite vaporization for the commercial production of fullerenes. As discussed in Section 1.4, combustion offers several advantages over vaporization including cheaper raw materials, lower operating costs, and adaptability to large-scale systems. Any combustion process proven to be efficient and productive in fullerene formation would need to be scaled-up for macroscopic production.

Although the details of such a process were unfortunately not identified for pure fullerenes through the course of this work, it was observed that fullerenic soot (soot containing fullerenic character) could be efficiently made through combustion. The prevalence of carbon black (conventional flame-generated particulate carbon synthesized in industry with no fullerenic content) to make rubber, tires, inks, and other products [130] indicates a potential niche market for fullerenic soot may exist if it can prove to be economically competitive and superior in desired bulk properties, which it has for certain applications [82].

To evaluate the cost of a large-scale fullerenic soot plant, an incremental cost model was developed based on existing carbon black technology. This model examines the extra cost incurred by taking an existing carbon black production line and converting to make fullerenic soot. In this manner, it becomes relatively straightforward to determine the competitiveness of fullerenic soot with conventional carbon black.

Sensitivity analyses performed on the model indicate that fullerenic soot will be more expensive than carbon black, even given optimal operating conditions and expected increases in efficiency. However, the price may be low enough to justify production for separate applications and markets. Limitations on the model, though, leave room for and necessitate a more detailed study before the same conclusions can be drawn for a pure fullerene product. More study is also needed to identify optimal parameter settings before a commercial fullerenic soot plant can actually be built. 


\subsection{Model basis}

The economic cost model was based on current carbon black production technology as outlined by Donnet et al. [130]. 'The model involved analyzing Donnet's plant $^{\dagger}$ and determining changes necessary to convert it into a fullerenic soot producing plant. The costs of these changes were estimated and totaled to provide the incremental cost of producing fullerenic soot above and beyond the cost of producing carbon black. The main reasons for doing such an analysis instead of estimating the cost from scratch were that the two plants are nearly identical making many of the calculations redundant and information concerning the cost of a carbon black plant is not readily available.

A schematic of Donnet's plant is shown in Figure 7-1. In this figure, the reactor (1) is fed with fuel feedstock, hot air, and natural gas fuel. The gas and the air are fed upstream of the fuel feedstock, which usually consists of liquid hydrocarbon oils. This configuration allows for the combustion of the gas, which provides the energy and temperature for the subsequent product formation and also consumes most of the available oxygen. This leads to the pyrolysis of the feedstock into carbon black.

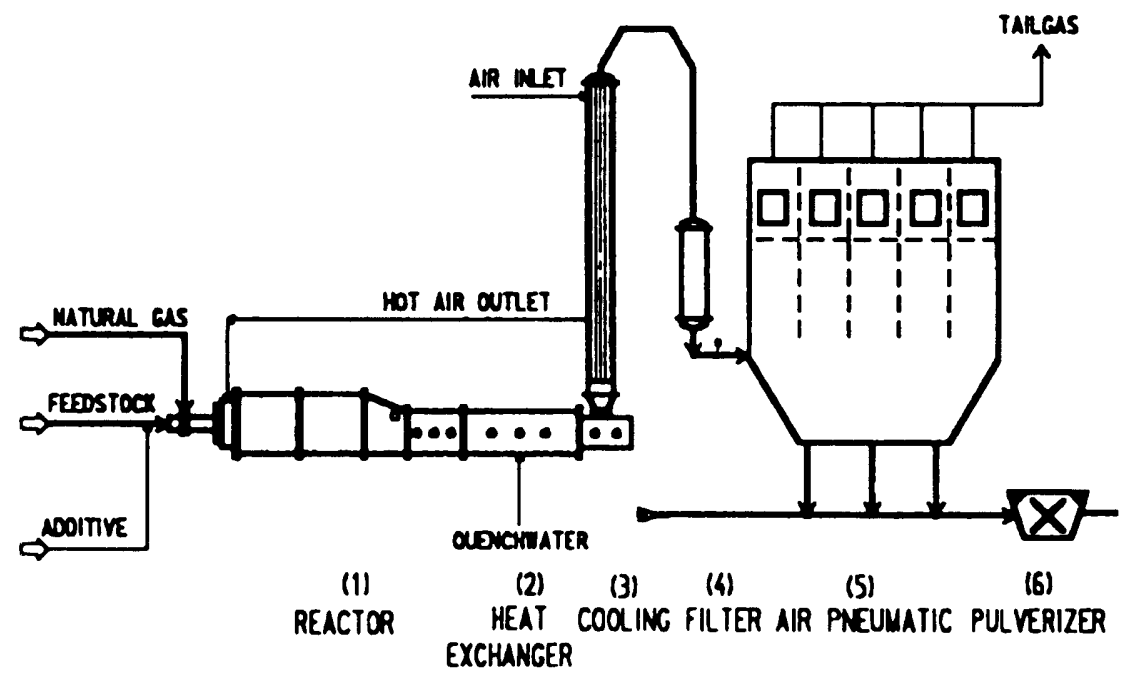

Figure 7-1. Schematic of commercial carbon black plant

\footnotetext{
* All the information in this section is taken from the Donnet et al. reference cited here. Thus, this citation is meant cover the entire section.

'For the remainder of the chapter, the plant described by Donnet et al. will be referred to as 'Donnet's plant' for clarity.
} 
Further downstream, the reaction is quenched with water and the carbon black is formed and cooled. The heat exchangers (2) provide further cooling (3), and the carbon black is sent to a filter (4) where it is separated from the tail gas. The filtered product is then conveyed pneumatically (5) to the pulverizer where all particles are reduced to an acceptable size with a hammer mill (6). Although not shown in Figure 7-1, the carbon black continues on through a pelletizer, drier, another filter, and size separator before being discharged into a packing unit or a loading station.

The major parameters of Donnet's plant that are of importance in the cost model analysis are listed in Table 7-1 along with their numerical values. In Table 7-1, $\mathrm{Nm}^{3}$ stands for normal cubic meters, that is volume at normal room temperature and pressure.

Table 7-1. Major parameters for Donnet's carbon black production plant

\begin{tabular}{|c|c|}
\hline Parameter & Value \\
\hline Plant product capacity & $3000 \mathrm{~kg} / \mathrm{hr}$ \\
\hline Soot yield & $0.70 \mathrm{~g}$ soot $/ \mathrm{g}$ carbon fed \\
\hline Air feed rate & $10,000 \mathrm{Nm}^{3} / \mathrm{hr}$ \\
\hline Air consumption factor $(k)$ & $65 \%$ \\
\hline $\begin{array}{l}\text { Stoichiometric air consumption } \\
\text { factor for methane }\left(f_{s}\right)\end{array}$ & 9.55 \\
\hline Gas feed rate & $681 \mathrm{Nm}^{3} / \mathrm{hr}$ \\
\hline Feedstock feed rate & $4285 \mathrm{~kg} / \mathrm{hr}$ \\
\hline Wet tail gas & $20,000 \mathrm{Nm}^{3} / \mathrm{hr}$ \\
\hline Reactor pressure & 760 Torr \\
\hline
\end{tabular}

The only major modifications to Donnet's plant that were made in this approximate representation of fullerenic soot production were operation of the reactor at low pressure and the use of liquid benzene as the fuel feedstock. These changes, along with other assumptions taken in the model and adjustments to the parameters in Table 7-1 are further discussed in the next section. A more detailed description of Donnet's plant can be found in the first chapter of the Donnet reference for this section. 


\subsection{Assumptions}

In developing the cost model, several simplifying and clarifying assumptions had to be made. These assumptions fall into two major categories: those taken in identifying what modifications would be needed to convert Donnet's plant into an approximate representation of a fullerenic soot plant; and those taken with respect to the operating conditions and production capabilities of the new plant. This work appreciates that erroneous or misguided assumptions can often lead to inaccurate results and model predictions. However, it is believed that all of the assumptions listed here are reasonable approximations that do not alter the outcomes of the model or the conclusions drawn from it.

\subsubsection{Incremental changes and additions}

Converting a carbon black plant into a fullerenic soot plant first and foremost requires that the operating pressure in the reactor be dropped to pressures suitable for fullerene formation - generally in the 20-100 Torr range. To achieve this, it was assumed that high-capacity vacuum pumps would be used but that the filtering of the soot product would be accounted for upstream and separately from these pumps. A filtering system is already utilized to collect carbon black in the current configuration [130] making it unnecessary to add this to incremental cost figures.

In addition, low-pressure operating requires the system to be sealed and tight-fit to prevent air leaks, which could alter the yield, and to minimize the power needed to keep the system under pressure. However, Donnet et al. indicate that carbon black reactors are already sealed tight for insulation purposes to prevent thermal heat loss and to allow material flow to be closely controlled [130]. Again, this needs not be accounted for in the cost model.

Finally, the secondary fuel feedstock used for the majority of the soot production is assumed to be benzene instead of the more conventional oils as studies have shown that benzene fuel, in terms of organic materials, is the most favorable for fullerene formation $[32,33]$. Although the identity of the fuel was altered, it was not necessary to modify the feeding system as the carbon black fuel oil is also a liquid feedstock and has physical properties similar to those of benzene. 


\subsubsection{Operating parameters}

Many assumptions were made with respect to the operation and specific details of the fullerenic soot plant. These assumptions, along with the justification and explanation of each, are listed below:

1. The production rate of soot is equal to the typical capacity of a carbon black plant. To make cost figures more comparable, the output of fullerenic soot was set equal to that of Donnet's plant at 3 metric tons/hour.

2. The yield of soot is equal to $3 \%$ of the carbon fed. Although this is significantly smaller than Donnet's yield of $70 \%$, it is equal to the that observed in the premixed sooting flame experiments discussed earlier in this work, and was confirmed by thermodynamic equilibrium calculations performed by STANJAN (see Appendix 3 for the results of both calculations). This yield provides a more realistic assessment of plant performance.

3. Primary flame feed gas is pure methane. Although natural gas is generally used for this purpose, methane was assumed to simplify calculations. Given that methane comprises about $85 \%$ of natural gas anyway, this assumption seems reasonable.

4. Volume flows into the reactor are scaled up to compensate for the lower operating pressure. Since it is desired to keep the production rate constant, mass flows must be maintained at present levels. However, this requires the volume flows to scale inversely with pressure and affects the size of the reactor.

5. The air and gas feed rates for the primary flame are identical to Donnet's plant. The primary flame is not used for soot production but mainly energy generation so it is safe to assume that this flame will not be altered.

6. The amount of conventional wet tail gas in the effluent is equal to the 20,000 $\mathrm{Nm}^{3} / \mathrm{hr}$ that is in the carbon black plant. Although there will be much more tail gas in the fullerenic soot plant due to the low yield, the effect of this is isolated and calculated separately as a tail gas resulting from non-yield fuel. 
Independent of the excess fuel, it is thought the tail gas would be similar in volume and composition to the current effluent.

7. The reactor temperature is similar to the carbon black reactor temperature. Given the similarity of the two systems and the difficulty of predicting temperatures in a theoretical combustion setup, it is reasonable to assume the temperature would not change appreciably between the two plants. This eliminates the temperature from consideration in reactor volume calculations.

8. The furnace tail gas is ideal and undergoes adiabatic compression across the vacuum pump. The ideal gas law is still valid for gases in the pressure and temperature ranges seen here so it can be applied without consequence. The adiabatic assumption is less solid but given that the entire system is insulated and the gases will cool prior to the pump, heat loss should be minimal.

9. Vacuum pump efficiency is $80 \%$. This is an efficiency typically seen in industrial-sized pumps for converting electrical energy into pumping power.

10. The heat of combustion of conventional tail gas is $2500 \mathrm{~kJ} / \mathrm{m}^{3}$ and that of tail gas due to low product yield is $3141 \mathrm{~kJ} / \mathrm{mol}$. The conventional tail gas energy is supplied Donnet et al. [130], while the low yield tail gas energy is calculated from a rough approximation that the heating value of leftover feedstock is in the same proportion to the initial heating value of the fuel as the difference between the stoichiometric oxygen rate and the amount of oxygen fed is to the stoichiometric oxygen rate. This relationship is shown in Equation 7-1:

$$
\frac{[\text { TailGasHeatingValue }]}{[\text { FeedstockHeatingValue }]}=\frac{O_{2, \text { stoich }}-O_{2, \text { fed }}}{O_{2, \text { stoich }}}=1-\phi^{-1}
$$

where $\phi$ is the equivalence ratio of the flame. The tail gas consists mostly of light gaseous species meaning that condensation and coking should be minimal. It is appreciated that the value computed is significantly higher than what would be expected for combustible tail gas. However, this is an artifact of the extremely low soot yield assumed in the calculation - meaning the excess energy from the high benzene flow is assumed to be passed on to the 
tail gas. A more accurate value is needed but the absence of experimental data at such yields prevents such a calculation.

11. The conversion efficiency of electricity generation is $40 \%$. This is a typical observed efficiency for converting fuel energy into electricity.

12. The cost of electricity is broken down into fuel cost and generation cost based on industrial electricity costs for southern states. The plant would most likely be located in the south where electricity is the cheapest. The fuel cost is based on a fuel price of 50\& per gallon [131] with an energy of 160,000 BTU per gallon and a $40 \%$ efficiency - all industry norms.

13. The capital equipment lifetime (vacuum pump and new reactor) is ten years with operation during 24 hours per day for 350 days per year. These are also accepted industry figures for capital usage and duration. These values provide a project lifetime basis for cost comparison.

As mentioned, these parameter assumptions will all affect cost predictions. However, with the justifications provided, it is believed that corresponding effect of any inaccuracies will be minimal. As a confirmation, where possible, the values were allowed to vary in a specified range to test the sensitivity of the price to certain parameters. The results of the most significant outcomes are discussed in the sensitivity analyses part of the next section.

\subsection{Cost model predictions}

The cost model was constructed in Excel and used to make predictions as to the final cost of producing fullerenic soot. Calculations were made both with base case values representing most likely or current figures and with certain parameters varied in a specified range to give sensitivity information. A reproduction of the spreadsheet for the base case predictions is shown in Appendix 4. A text explanation of the logic and calculations behind the lines in the model is also given in Appendix 4 following the spreadsheet. All of the dollar and cent values given here are adjusted to Year 2000 dollars. The results indicate that fullerenic soot can be competitive with conventional carbon black given the right conditions and advances in technology. 


\subsubsection{Base case cost estimate}

The base case cost model shows that the incremental cost to produce fullerenic soot come to $\$ 6.543$ per pound. Given that the current cost of carbon black is $\$ 0.35$ per pound, it seems that fullerenic soot could not be commercially competitive under such a setup. Possibilities for reducing this cost are discussed in the next section. The cost is broken down into its individual components in Table 7-2.

Table 7-2. Base case incremental costs for fullerenic soot production

\begin{tabular}{|l|c|c|}
\hline \multicolumn{1}{|c|}{ Component } & Absolute cost (\$) & Cost per pound (\$/lb) \\
\hline Reactor cost & 311,702 & 0.0006 \\
\hdashline Fuel cost & $43,146 / \mathrm{hr}$ & 6.524 \\
\hdashline Pumps cost & 546,933 & 0.0010 \\
\hdashline Power cost & $118 / \mathrm{hr}$ & 0.0178 \\
\hline Total & & 6.543 \\
\hline
\end{tabular}

As can be seen from Table 7-2, 99.7\% of the total incremental cost comes from the vast amounts of relatively expensive benzene fuel that must be fed to the system to compensate for the low yield in the system. This indicates that the cost can be dramatically reduced simply by using a cheaper fuel and/or improving the product yield. Both of these options are explored further in the following section.

The cost breakdown also shows that the capital costs (the pumps and the reactor) are negligible in the final cost, as is often the case with capital equipment. This means that neither the cost of the pumps required to come down to pressure nor the size of the reactor are limiting factors in fullerenic soot production. Prior to the construction of this model, these had been concerns as it was believed that the lower pressure would result in massive volumes of gas needing to be reacted and pumped away. The results suggest that the reactor can be as large as necessary and as many pumps as necessary can be installed without adversely affecting the final soot price.

Finally, it is also seen that electricity consumption is not an insurmountable cost. Again, this was an early concern as large pumps require large energy inputs. It is 
recognized that the energy requirement here is indeed quite big, but it is not unmanageable. Additionally, there is potentially 50 times more energy in the effluent tail gas than is required to run the pumps. Although not accounted for in this model, this energy could be sold or used in other parts of the plant to help offset fuel costs.

\subsubsection{Sensitivity to parameter adjustments}

Many parameters in the model were adjusted to elucidate their effect on the final product price but only four parameters were found to noticeably change the final values. These four parameters were soot yield, fuel price, reactor pressure, and pump lifetime. Of these, only two - soot yield and fuel price - were found to affect the price by more than a couple of cents per pound. In fact, adjustments to these two variables dropped the price to something that can be competitive with commercial carbon black.

Soot yield in the cost model was varied between $3 \%$ and $60 \%$. This range was chosen because the yield couldn't be much lower than it already is and while $60 \%$ is much higher than we can hope to achieve, extending the range gives us a good indication of the trends. The fuel price was varied between $\$ 0.50$ and $\$ 1.50$ per gallon. Again, at $\$ 1.50$, benzene is one of the most expensive organic fuels so it represents the top of the scale. Other organics can be even cheaper than $\$ 0.50$ but the chosen range is sufficient for our purposes. The results of the sensitivity analyses with respect to soot yield and fuel price are shown in Figure 7-2.

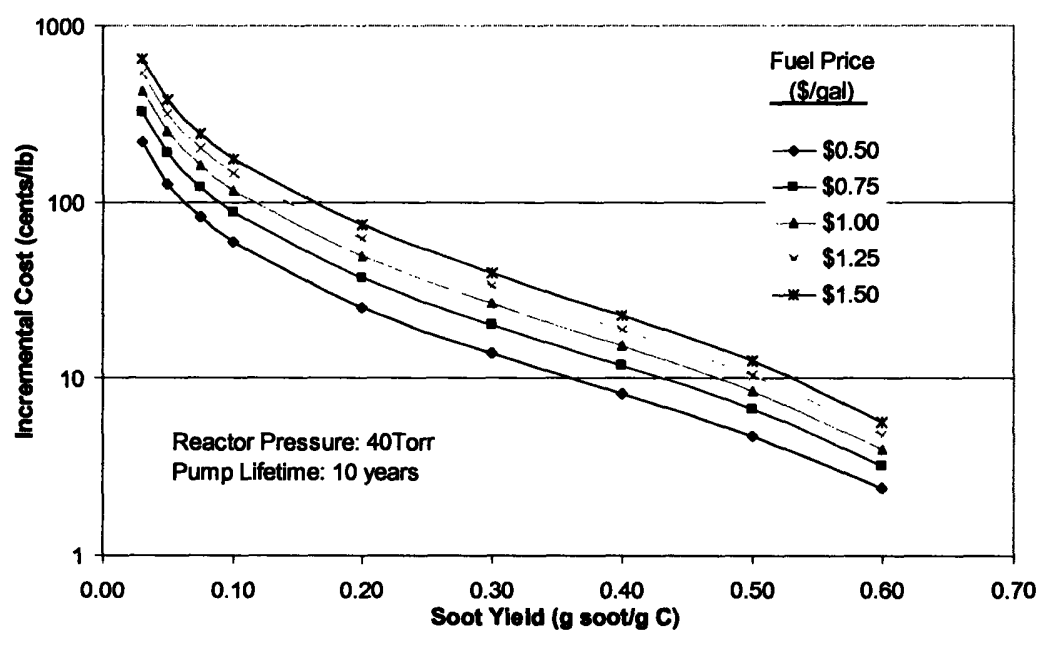

Figure 7-2. Senstivity of incremental fullerenic soot cost to soot yield and fuel price (reactor pressure $=40$ Torr and pump lifetime $=10$ years) 
For the data in Figure 7-2, the reactor pressure was kept constant at 40 Torr and the pump lifetime was fixed at 10 years. As can be seen, improving the fuel price alone can drop the price to $\$ 2.19$ per pound - only $1 / 3$ of the original cost. Bigger savings can be realized by increasing the yield even slightly. As shown, a yield of just $20 \%$, which seems feasible, could potentially drop the cost to just $25 \notin$ per pound, bringing it into a range where it can competitive with carbon black. Of course, increasing the yield further can bring the cost down to just a few pennies per pound. Such an extreme scenario seems highly unlikely as there would be tradeoffs with product quality (which are discussed in the Section 7.5) but the results do indicate that the incremental fullerenic soot price need not stay at $\$ 6.54$ per pound.

Sensitivity analyses with respect to reactor pressure and pump lifetime are shown in Figure 7-3. In these analyses, the reactor pressure is varied between 10 and 90 Torr and the pump lifetime from 4 to 12 years. Again, these ranges were chosen because they represent feasible parameter values sufficient to illustrate their respective effects. The soot yield and fuel price in these data were kept at $3 \%$ and $\$ 1.50$ per gallon, respectively.

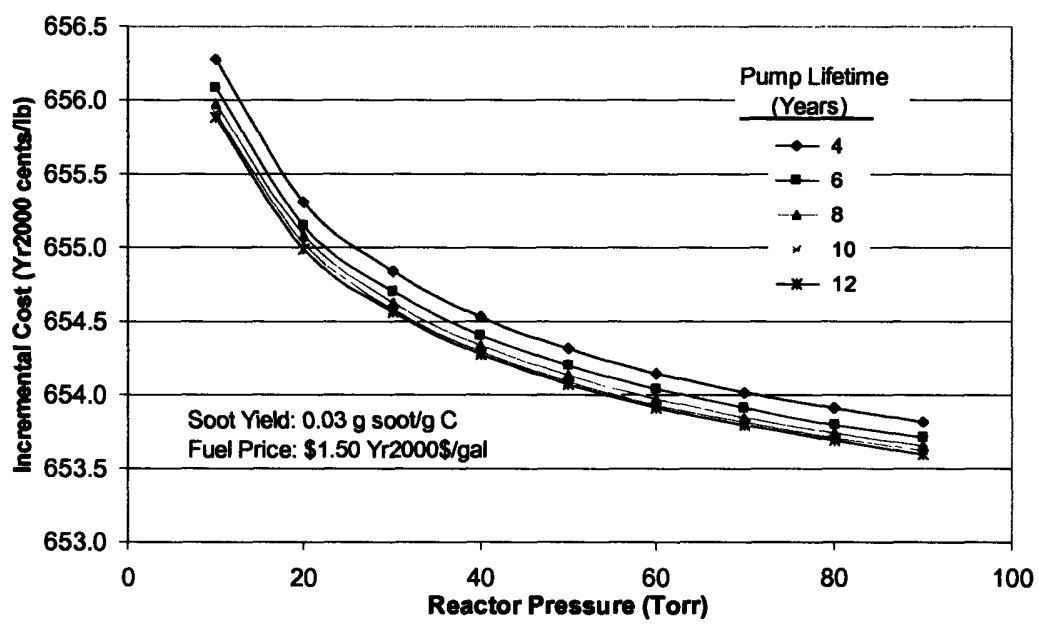

Figure 7-3. Senstivity of incremental fullerenic soot cost to reactor pressure and pump lifetime (soot yield $=3 \%$ and fuel price $=\$ 1.50$ per gallon)

As can be seen in Figure 7-3, changing the pump lifetime alters the price by less than a penny while the reactor pressure variations have a maximum effect of only three cents. Compared to the absolute price, these changes are insignificant.

To test if this was still the case at a lower absolute price, these analyses were performed again using a soot yield of $20 \%$ and a fuel price of $\$ 0.50$ per gallon. It was 
thought that the effect of pressure and lifetime may be amplified enough at a lower overall cost to make the relative changes significant. If so, knowing the magnitude of the effects would be helpful in determining the best possible line conditions. The results of this sensitivity analyses are presented in Figure 7-4.

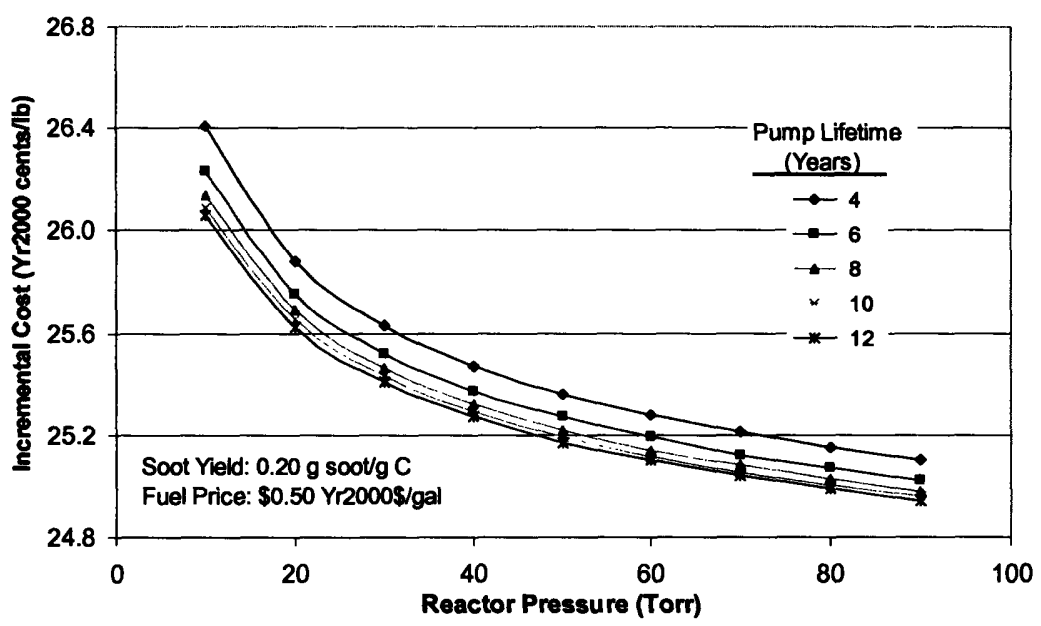

Figure 7-4. Senstivity of incremental fullerenic soot cost to reactor pressure and pump lifetime (soot yield $=20 \%$ and fuel price $=\$ 0.50$ per gallon)

As can be seen in Figure 7-4, the sensitivity to both pump lifetime and reactor pressure actually decrease slightly. In fact, the total variation in price is now only $1.5 \phi$. At the lower absolute price, this variation does comprise a greater percentage of the price but is still not enough to warrant any serious concern when the designing the fullerenic soot production system. In fact, the most important result from Figures 7-2 and 7-3 is that there exists a large degree freedom when choosing the pressure at which to operate the system. This becomes important as soot yield, one of the parameters identified as being of great importance, is often proportionally related to the operating pressure. This effect is discussed in more detail in the Section 7.5.

\subsection{Model limitations}

As with any theoretical model, there a number of factors not taken into account that could affect the final model results if included and should be kept in mind when examining them. The major factors not accounted for in this model can be divided into the two categories of making the final product more expensive and making it less expensive. While the factors in the former category outnumber those in the latter, it 
seems that there are enough of both to prevent a prediction on the final effect on price if all are considered simultaneously.

Factors that could increase cost include maintenance costs and associated labor, extra physical space required, and a method for efficiently changing the product filter at low pressure. The additional pumps and larger reactor will require regular maintenance and servicing to remain operational. Depending on reliability, these costs could be nontrivial. Similarly, the additional capital and low-pressure conditions may require another operator to oversee line operation, which could drive up labor costs. The increased capital will also require more physical space and thus more land. Although land is relatively cheap in the south where the plant would be built, it could still add to the overall cost. Lastly, changing the filter for product collection will also incur a cost. The carbon black filter system (described in Section 7.1) will not be suitable because of the subatmospheric operating pressure. A new system would need to be designed to account for the low system pressure and minimal pressure drop across the filter. While it is believed it can be similar to current technology, an extra cost will almost certainly be realized.

A cost savings could be seen, though, if several other factors are also considered. These factors include profits from the sale of excess energy in the tail gas and also increased revenue from the higher price that fullerenic soot will presumably command in the market. A rough approximation of the tail gas energy profits using base-case data in the model comes to over $\$ 11,000$ per hour which offsets over $25 \%$ of the fuel cost. Additionally, if this energy could not be sold, it may be feasible to recycle the excess benzene back to the feedstock thereby offsetting the fuel costs in a different manner. It's quite possible that with enough recycling, the soot yield could increase without any other advances in efficiency. The increased revenue is difficult to estimate as it would be based on market conditions but a somewhat higher price would have to be charged to turn a profit on a more expensive product.

As mentioned, all of these factors will affect the bottom line of fullerenic soot production and all need to be carefully evaluated before the final true incremental cost can be known. However, it is thought that these factors will not alter the conclusions drawn from the data presented above. In any case, the model still highlights some important implications and recommendations for the emerging fullerenic soot industry. 
Turning our attention for a moment to a pure fullerene product, this model does not make any estimations for the cost associated with extracting fullerenes from the fullerenic soot. In current commercial production of pure fullerenes, this is often a significant contributing factor to fullerene price. A detailed study of a large-scale separation process would need to be considered before any of the results here could be extended to pure fullerenes production.

\subsection{Implications for fullerene price and fullerenic soot industry}

There are two major conclusions that can be drawn from the results of the cost model: fullerenic soot produced from combustion can be competitive with conventional commercial carbon black; and, more importantly, purified fullerenes produced from this method can be significantly cheaper than those currently sold commercially.

Considering first the pure fullerene price, conservative estimates of production costs and fullerene selectivity reveal figures that are substantial improvements over the current price. Taking the base-case scenario for soot production $(\$ 6.54 / \mathrm{lb})$ and assuming a $10 \%$ selectivity for $\mathrm{C}_{60}$ (observed in the diffusion flames in Section 3.2.2.1), gives a base fullerene price of about $\$ 65 / \mathrm{lb}$. Taking another conservative estimate that extracting and purifying the fullerenes will cost the same as production [111], a final fullerene price tag of $\$ 130 / \mathrm{lb}$ is estimated, which is consistent with previous analysis [108].

Given the current commercial price of approximately $\$ 9000 / \mathrm{lb}[106]$, this estimated figure represents a tremendous price reduction of over $98 \%$. This estimate is more striking given that it was calculated using fairly conservative production figures. The price could be reduced even further if more efficient scenarios are realized during production. Selling fullerenes at such low prices will greatly expand their market in both academic and industrial settings as $\mathrm{C}_{60}$ would become cheap enough for use in consumer goods as well as research. Consequently, it appears that combustion is well-poised to become a suitable alternative to the current commercial production method.

Now considering the production of fullerenic soot, some important studies need to be completed before it can be competitive with carbon black. The most important of these studies is to determine the effect of pressure on soot yield and fullerenic content. In general, as pressure rises, soot yield also rises but fullerenic content falls resulting in a 
frustrating trade-off. Similarly, using a cheaper fuel can also drive down cost but again, there is an undesirable trade-off. Fuels other than benzene, while cheaper, tend to produce soot with lowered fullerenic content and altered characteristics. Determining the cheapest fuel and highest soot yield possible that still make desirable soot will hopefully lower costs. Additionally, other methods exist for improving the soot yield. These methods, such as using different fuels, changing the character of the primary flame, or adjusting the reactor size and residence time, all need more testing in an industrial setting. Most likely, trade-offs will again exist between yield and fullerenic content so a detailed study will be valuable in identifying the optimal operating conditions.

Applications for fullerenic soot must also materialize to make its production economically desirable. It could compete directly with carbon black if the price is sufficiently low. One example is its use in the formation of vulcanized rubber for tires, where its bulk properties are superior to those of carbon black as shown in a Goodyear patent [82]. This represents a huge market as rubber is by far the single greatest use of carbon black worldwide. Carbon black is also made in various grades and prices and fullerenic soot could steal market share from the more expensive grades. However, fullerenic soot can fill niche markets unavailable to conventional carbon black. These markets include serving as an adsorbent for hexavalent chromium and metal cations [132, 133] and dioxins [83]. In aggregate, these applications could create a market large enough to absorb the production of as much soot as is presented here. In the absence of such a demand though, the plant could be scaled down, to as little as $10 \%$ of the current size, without significant cost increases.

The fact that fullerenes and fullerenic soot are produced concurrently is, while detrimental to fullerene production, actually a boon to soot production. Even after fullerene extraction, the soot remains fullerenic with significant fullerene characteristics. This implies that the profit realized from fullerene sales could be used to subsidize the fullerenic soot, thus making it even cheaper. If the costs of production can be recovered from fullerene sales alone, then the soot could be sold cheaper than carbon black and potentially take the entire market. Given this possibility, it seems that this production method can be economically competitive not only with commercial fullerene production but also with conventional carbon black. 


\section{Conclusions}

The work presented in this thesis has resulted in conclusions that have implications for industry as well as for further fundamental research. While not all of the conclusions were expected, or even favorable, they all nonetheless have added to the database of fullerene and nanostructure formation through combustion. The major conclusions of the thesis are summarized as follows.

\section{- Diffusion flames}

- Both laminar and turbulent-like diffusion flames are capable of making fullerenes but only laminar flames can make them in a predictable and documented manner.

- In laminar flames, fullerene concentration increases with residence time until just beyond the stoichiometric surface, after which it decreases.

- The evolution of carbon structure curvature in laminar flames follows trends similar to those of fullerene concentration.

- Nanostructures are found at very long residence times in laminar flames.

- Fullerenes may be consumed by soot in situ.

- Increasing dilution in laminar flames leads to competing effects.

- For a constant cold gas velocity, shorter flames and hotter flames tend to have higher fullerene concentration.

- The fullerene-producing region in turbulent flames is much larger than in laminar flames but yields are much smaller.

- Making predictions for fullerene production in turbulent flames cannot be done with any accuracy.

- Diffusion flames are promising for fullerene production and laminar flames are more desirable than turbulent flames.

- Premixed flames

- Secondary fuel in two-stage flames has an adverse effect on fullerene formation. 
- Secondary fuel is creating pyrolysis conditions similar to those seen in the very early stages of the Mckinnon flame, where pyrolysis is also occurring.

- Combustion is necessary for fullerene production, either through precursor generation or overcoming a formation barrier.

- Two-stage flames with pure fuel secondary feeds are not suitable for fullerene production.

- Coexistence of soot and fullerenes in the one-stage Mckinnon flame indicates that fullerenes are not consumed by soot in situ.

- Understanding formation and consumption mechanisms better would help in designing flames more promising of fullerenes.

- Fullerene reaction with soot

- Fullerene $\mathrm{C}_{60}$ reacts with soot in the temperature range 1023-1273 $\mathrm{K}$ in accordance with first-order kinetics.

- Collision efficiency and activation energy are consistent with a reaction controlled by surface diffusion or a heterogenous reaction.

- The reaction rate extrapolated to flame conditions is too small to account for the observed rate of fullerenes consumption in a fullerene-forming flame.

- Mechanisms other than fullerene-soot reaction must be responsible for fullerene consumption in flames

- Fullerenes tethered to carbon black particles

- HRTEM analyses of samples containing fullerenes $\left(\mathrm{C}_{60}\right)$ tethered to carbon black show an order-of-magnitude greater occurrence of fullerenetype structures than a similar analysis of pure carbon black.

- Diameter size distributions of the tethered samples show a strong peak at the diameter of $\mathrm{C}_{60}$ while the carbon black sample does not.

- Circular structures seen in HRTEM images of combustion-generated soot are in fact fullerene molecules. 
- Fullerenes can indeed be directly observed by HRTEM analysis.

- Cost modeling of a commercial fullerenic soot plant

- Increasing soot yield is the most important factor in driving down costs of fullerenic soot.

- The price of fuel is also quite significant in determining the soot price; using a cheaper fuel than benzene could result in massive savings.

- Capital costs and power costs are negligible in the final product price.

- By minimizing trade-offs, fullerenic soot can be competitive on a cost basis with conventional commercial carbon black.

- Pure $\mathrm{C}_{60}$ can be produced inexpensively and sold at a cost saving of up to $98 \%$ compared to current commercial production methods.

- Overall

- Combustion has not yet been optimized for fullerene production

- The combustion process for the formation of fullerenes from organic fuels is necessary but not yet well understood.

- Much more study is needed to effect a better understanding of fullerene formation and consumption mechanisms in flames.

- Combustion holds immense promise to be a much cheaper and more efficient alternative to the current method of commercial fullerene production. 


\section{Recommendations}

There are many studies that can be performed to build on and complement the conclusions presented in the previous chapter. Possibilities for future work include experimental flame studies to identify conditions of optimal fullerene production as well as more specific mechanistic studies to effect a detailed understanding of fullerene interactions. There also exist several opportunities for industrial research to provide more profitable commercial fullerene production plants. The recommendations arising from this thesis work are outlined below.

- Characterize a greater range of conditions for fullerene-forming flames to identify optimal fullerene production conditions both in terms of fullerene yield and fullerene price. Both diffusion and premixed flames need more study under varying parameters such as higher pressure, greater dilution, and more heavily sooting. In addition, systems with completely different flame configurations should be tested. These configurations can include:

- Thermal insulation surrounding the flame to minimize radiation loss and increase flame temperature. Preliminary experiments with insulation were performed as part of this thesis work but the results were inconclusive.

- Separating the fullerenes from the soot in situ through the use of an electric field or a soot filter to prevent any sort of consumption. With such separation, the soot/fullerene contact could be reduced essentially to zero, thereby eliminating any consumption by soot or soot byproducts.

- Two-stage flames with a premixed secondary injection consisting of fuel, oxygen, diluent, and/or fullerene precursor species rather than just fuel.

- Significantly increased residence times through flames in microgravity.

- Characterize fullerene-forming flames for PAH to identify fullerene precursor species. As mentioned, there is significant disagreement about fullerene formation mechanisms and identifying fullerene precursors would not only help reconcile these disagreements but also help with modeling efforts (discussed below). 
- The last configuration above about microgravity flames is actually more geared toward understanding mechanistic influences in fullerene formation. Such flames will test the effect of buoyancy independent of pressure, which will be helpful, but many more reactions and interactions need to be studied. Reactions that both produce and consume fullerenes should be studied to determine their reaction rate coefficients including activation energies and collision efficiencies. Studying these reactions in a detailed manner will eventually produce a database of rate constants pertinent to fullerenes much like those that exist for reactions involving PAH. Knowing fullerene precursors will indicate which reactions to study. Although it may be necessary to study individual reactions to determine these fullerene precursors, mechanistic studies will give a strong indication of which reactions and species are most important.

- Once these reactions have been studied, they can be used for modeling purposes to create simulations predictive of fullerene concentrations. Such models can be compared to flame characterizations for fullerene verification and then used to estimate fullerene formation in theoretical flames, greatly improving the likelihood of optimizing fullerene production. The development of a kinetic model to predict fullerene formation is vital to the intelligent design of flames for increased fullerene production.

- Characterize soot structure evolution in a more detailed manner in premixed flames. It is unknown whether similar conclusions for soot in premixed flames can be drawn as were drawn for diffusion flames. Knowing if soot structure follows the same pattern as fullerene concentration, like it does in diffusion flames, would have important implications about both the fullerene mechanism and nanostructure formation.

- In conjunction with the preceding recommendation, soot structure evolution should be studied during the heat treatment of combustion-generated soot. Such 
studies can provide insight to soot behavior in flames and again have mechanistic and nanostructure formation implications. If possible, it would be preferable to do this in an HRTEM microscope so that the same region of soot material can be continually monitored as it undergoes changes due to thermal treatment.

- Identify conclusively the presence of fullerenes smaller than $\mathrm{C}_{60}$ in HRTEM images. Such fullerenes have adjacent pentagons and their presence in soot would significantly alter the soot's bulk properties. Now knowing that $\mathrm{C}_{60}$ can be seen in HTREM, these species should also be verified. It would also be useful to identify a spectroscopic characterization method other than HRTEM for small fullerenes but no such method is currently available.

- Bulk properties of fullerenic soot should be tested to determine its utility in industrial applications. Although it has been shown that fullerenic soot can compete economically with certain carbon blacks and fullerenes from other methods, the desirability of the fullerenic soot must be demonstrated before a significant market will materialize.

- Other industrial research should be performed to determine the acceptable balances in trade-offs between fullerenic soot quality and price. These trade-offs exist, for example, in choosing the furnace fuel to produce the soot or in choosing the operating pressure as higher pressure leads to more soot but less fullerenes. This ties in also to bulk property testing as all qualities of fullerenic soot should be analyzed to determine their practicality.

- A pilot-plant scale fullerene and fullerenic soot facility should be constructed to perform this research. A pilot-plant will provide an inexpensive test bed to test fullerene production and investigate soots that would be expected to be produced in a commercial-size plant. Such plant will ultimately determine the feasibility of using combustion to synthesize fullerenes and fullerenic nanostructures. 


\section{References}

[1] Kroto, H.W., Heath, J.R., O'Brien, S.C., Curl, R.F., and Smalley, R.E. "C 60 : Buckminsterfullerene", Nature 318:162-163, (1985).

[2] Jarrold, M.F. "The smallest fullerene", Nature 407:26-27, (2000).

[3] Heath, J.R. "C 60 's smallest cousin", Nature 393:730-731, (1994).

[4] Prinzbach, H., Weiler, A., Landerberger, P., Wahl, F., Worth, J., Scott, L.T., Gelmont, M., Olevano, D., and Issendorff, B.V. "Gas-phase production and photoelectron spectroscopy of the smallest fullerene, $C_{20}$ ", Nature 407:60-63, (2000).

[5] Chai, Y., Guo, T., Jin, C., Haufler, R.E., Chibante, L.P.F., Fure, J., Wang, L., Alford, J.M., and Smalley, R.E. "Fullerenes with Metals Inside", Journal of Physical Chemistry 95:7564-7568, (1991).

[6] Clemmer, D.E., Shelimov, K.B., and Jarrold, M.F. "Gas-phase self-assembly of endohedral metallofullerenes", Nature 367:718-720, (1994).

[7] Gillan, E.G., Yeretzian, C., Min, K.S., Alvarez, M.M., Whettern, R.L., and Kaner, R.B. "Endohedral Rare-Earth Fullerene Complexes", Journal of Physical Chemistry 96:6869-6871, (1992).

[8] Heath, J.R., O'Brien, S.C., Zhang, Q.L., Liu, Y., Curl, R.F., Kroto, H.W., Tittel, F.K., and Smalley, R.E. "Lanthanum Complexes of Spheroidal Carbon Shells", Journal of the American Chemical Society 107:7779-7780, (1985).

[9] Iijima, S. "Helical microtubules of graphitic carbon", Nature 354:56-58, (1991).

[10] Qin, L.-C., Zhao, X., Hirahara, K., Miyamoto, Y., Ando, Y., and lijima, S. "The smallest carbon nanotube", Nature 408:50, (2000).

[11] Sun, L.F., Sie, S.S., Liu, W., Zhou, W.Y., Liu, Z.Q., Tang, D.S., Wang, G., and Qian, L.X. "Creating the narrowest carbon nanotubes", Nature 403:384, (2000).

[12] Wang, N., Tang, Z.K., Li, G.D., and Chen, J.S. "Single-walled $4 \AA$ carbon arrays", Nature 408:50-51, (2000).

[13] Iijima, S. "The $\mathrm{C}_{60}$-Carbon Cluster Has Been Revealed!" Journal of Physical Chemistry 91:3466-3467, (1987).

[14] Kratschmer, W., Lamb, L.D., Fostiropoulos, K., and Huffman, D.R. "Solid C60: a new form of carbon", Nature 347:354-358, (1990).

[15] Haufler, R.E., Conceicao, J., Chibante, L.P.F., Chai, Y., Byrne, N.E., Flanagan, S., Haley, M.M., O'Brien, S.C., Pan, C., Xiao, Z., Billups, W.E., Ciufolini, M.A., Hauge, R.H., Margrave, J.L., Wilson, L.J., Curl, R.F., and Smalley, R.E. "Efficient Production of $\mathrm{C}_{60}$ (Buckminsterfullerene), $\mathrm{C}_{60} \mathrm{H}_{36}$, and the Solvated Buckide Ion", Journal of Physical Chemistry 94:8634-8636, (1990). 
[16] Bunshah, R.F., Jou, S., Prakash, S., Doerr, H.J., Isaacs, L., Wehrsig, A., Yeretzian, C., Cynn, H., and Diedrich, F. "Fullerene Formation in Sputtering and Electron Beam Evaporation Processes", Journal of Physical Chemistry 96:68666869, (1992).

[17] Chibante, L.P.F., Thess, A., Alford, J.M., Diener, M., D., and Smalley, R.E. "Solar Generation of the Fullerenes", Journal of Physical Chemistry 97:86968700, (1993).

[18] Fields, C.L., Pitts, J.R., Hales, M.J., Bingham, C., Lewandowski, A., and King, D.E. "Formation of Fullerenes in Highly Concentrated Solar Flux", Journal of Physical Chemistry 97:8701-8702, (1993).

[19] Hare, J.P., Kroto, H.W., and Taylor, R. "Preparation and UV/visible spectra of $\mathrm{C}_{60}$ and $\mathrm{C}_{70}$ ", Chemical Physics Letters 177:394-398, (1991).

[20] Wilson, M.A., Pang, L.S.K., Quezada, R.A., Fisher, K.J., Dance, I.G., and Willett, G.D. "Fullerene production in alternative atmospheres", Carbon 31:393-396, (1993).

[21] Peters, G. and Jansen, M. "A New Fullerene Synthesis", Angewandte Chemie: International Edition in English 31:223-224, (1992).

[22] Yoshie, K., Kasuya, S., Eguchi, K., and Yoshida, T. "Novel method for $\mathrm{C}_{60}$ synthesis: A thermal plasma at atmospheric pressure", Applied Physics Letters 61:2782-2783, (1992).

[23] Taylor, R., Langley, G.J., Kroto, H.W., and Walton, D.R.M. "Formation of $\mathrm{C}_{60}$ by pyrolysis of naphthalene", Nature 366:728-731, (1993).

[24] Beck, M.T., Dinya, Z., Dombi, A., Fetzer, J.C., Keki, S., Papp, L., Szabo, P.T., Szepvolgyi, J., and Zsuga, M. "Comparative Study of Carbonization of Toluene upon Heating, Electric Discharges, Silent Electric Discharges, and in Radiofrequency Plasma", in Fullerenes: Recent Advances in the Chemistry and Physics of Fullerenes and Related Materials, K.M. Kadish and R.S. Roff, Editors. 1994, Electrochemical Society: Pennington, New Jersey. p. 80-91.

[25] Petcu, S., Cauchetier, M., Armand, X., Vociu, I., and Alexandrescu, R. "Formation of Fullerenes in the Laser-Pyrolysis of Benzene", Combustion and Flame 122:500-507, (2000).

[26] Ebbesen, T.W. and Ajayan, P.M. "Large-scale synthesis of carbon nanotubes", Nature 358:220-222, (1992).

[27] Iijima, S. and Ichihashi, T. "Single-shell carbon nanotubes of 1-nm diameter", Nature 363:603-605, (1993).

[28] Bethune, D.S., Klang, C.H., de Vries, M.S., Gorman, G., Savoy, R., Vazquez, J., and Beyers, $R$. "Cobalt-catalyzed growth of carbon nanotubes with single-atomic layer walls", Nature 363:605-607, (1993). 
[29] Hatta, N. and Murata, K. "Very long graphitic nano-tubules synthesized by plasma-decomposition of benzene", Chemical Physics Letters 217:398-402, (1994).

[30] Vander Wal, R.L. and Ticich, T.M. "Comparative flame and furnace synthesis of single-walled carbon nanotubes", Chemical Physics Letters 336:24-32, (2001).

[31] Cheng, H.M., Li, F., Su, G., Pan, H.Y., He, L.L., Sun, X., and Dresselhaus, M.S. "Large-scale and low-cost synthesis of single-walled carbon nanotubes by the catalytic pyrolysis of hydrocarbons", Applied Physics Letters 72:3282-3284, (1998).

[32] Ahrens, J., Bachmann, M., Baum, T., Griesheimer, J., Kovacs, R., Weilmunster, P., and Homann, K.-H. "Fullerenes and their ions in hydrocarbon flames", International Journal of Mass Spectrometry and Ion Processes 138:133-148, (1994).

[33] Richter, H., Fonseca, A., Thiry, P.A., Gilles, J.-M., Nagy, J.B., and Lucas, A.A. "Combustion Synthesis of Fullerenes", MRS 1994 Fall Meeting, (1994).

[34] Richter, H., Fonseca, A., Gilles, J.-M., Nagy, J.B., Thiry, P.A., Rozenberg, R., de Hoffman, E., and Pasch, $\mathrm{H}$. "Effect of $\mathrm{HBr}, \mathrm{HCl}$, and $\mathrm{Cl}_{2}$ on Fullerene Formation in Benzene/Argon/Oxygen Flames", Carbon 34:317-326, (1996).

[35] Howard, J.B., Mckinnon, J.T., Makarovsky, Y., Lafleur, A.L., and Johnson, M.E. "Fullerenes $\mathrm{C}_{60}$ and $\mathrm{C}_{70}$ in flames", Nature 352:139-141, (1991).

[36] Chowdhury, K.D., Howard, J.B., and Vander Sande, J.B. "Fullerenic nanostructures in flames", Journal of Materials Research 11:341-347, (1996).

[37] Grieco, W.J. "Fullerenes and Carbon Nanostructures Formation in Flames", Ph.D. Thesis, Massachusetts Institute of Technology (1999).

[38] Howard, J.B., Chowdhury, K.D., and Vander Sande, J.B. "Carbon Shells in Flames", Nature 370:603, (1994).

[39] Heymann, D., Chibante, L.P.F., Brooks, R.R., Wolbach, W.S., and Smalley, R.E. "Fullerenes in the Cretaceous-Tertiary Boundary Layer", Science 265:645-647, (1994).

[40] Daly, T.K., Buseck, P.R., Williams, P., and Lewis, C.F. "Fullerenes from a Fulgurite", Science 259, (1993).

[41] Fang, P.H. and Wong, R. "Evidence for fullerene in a coal of Yunnan, Southwestern China", Materials Research Innovations 1:130-132, (1997).

[42] Fang, P.H., Zhou, X., Tao, R., Wang, Q., Mu, C., and Wu, X. "Fullerenes discovered in coal mines in Yunnan, China", Innovations in Materials Research 1:129-134, (1996). 
[43] Buseck, P.R., Tsipursky, S.J., and Hettich, R. "Fullerenes from the Geological Environment", Science 25:215-217, (1992).

[44] Becker, L., Bunch, T.E., and Allamandola, L.J. "Higher fullerenes in the Allende meteorite", Nature 400:227-228, (1999).

[45] Becker, L., Bada, J.L., Winans, R.E., Hunt, J.E., Bunch, T.E., and French, B.M. "Fullerenes in the 1.85-Billion-Year-Old Sudbury Impact Structure", Science 265:642-645, (1994).

[46] di Brozolo, F.R., Bunch, T.E., Fleming, R.H., and Macklin, J. "Fullerenes in an impact crater on LDEF spacecraft", Nature 369:37-40, (1994).

[47] Kroto, H.W., Allaf, A.W., and Balm, S.P. "C 60 : Buckminsterfullerene", Chemical Reviews 91:1213-1235, (1991).

[48] Foing, B. and Ehrenfreund, P. "Spectral fingerprints of Fullerene $\mathrm{C}_{60}{ }^{+}$detected in the interstellar medium", Solar System News 14:2-3, (1994).

[49] Becker, L., Poreda, R.J., and Bunch, T.E. "Fullerenes: An extraterrestrial carbon carrier phase for noble gases", Proceedings of the National Academy of Sciences 97:2979-2983, (2000).

[50] Becker, L., Poreda, R.J., Hunt, A.G., Bunch, T.E., and Rampino, M. "Impact Event at the Permian-Traissic Boundary: Evidence from Extraterrestrial Noble Gases in Fullerenes", Science 297:1530-1533, (2001).

[51] Becker, L., Poreda, R.J., and Bada, J.L. "Extraterrestrial Helium Trapped in Fullerenes in Sudbury Imapct Structure", Science 272:249-252, (1996).

[52] Zimmerman, J.A., Eyler, J.R., Bach, S.B.H., and McElvany, S.W. "'Magic Number" carbon cluster: Ionization potentials and selective reactivity", Journal of Chemical Physics 94:3556-3562, (1991).

[53] Yoo, R.K., Buscic, B., and Berkowitz, J. "Vacuum ultraviolet photoionization mass spectrometric study of $\mathrm{C}_{60}$ ", Journal of Chemical Physics 96:911-918, (1992).

[54] Lichtenberger, L.L., Nebesny, K.W., Ray, C.D., Huffman, D.R., and Lamb, L.D. "Valence and core photoelectron spectroscopy of $\mathrm{C}_{60}$, buckminsterfullerene", Chemical Physics Letters 176:203-208, (1991).

[55] Pope, C.J. and Howard, J.B. "Thermochemical Properties of Curved PAH and Fullerenes: A Group Additivity Method Compared with MM3(92) and MOPAC Predictions", Journal of Physical Chemistry 99:4306-4316, (1995).

[56] Cioslowski, J., Rao, N., and Moncrieff, D. "Standard Enthalpies of Formation of Fullerenes and Their Dependence on Structural Motifs", Journal of the American Chemical Society 122:8265-8270, (2000). 
[57] Cataldo, F. "On the enthalpy of formation of the most known carbon allotropes", Fullerene Science and Technology 5:1615-1620, (1997).

[58] Doome, R.J. "New evidences for the anomalous temperature-dependent solubility of $\mathrm{C}_{60}$ and $\mathrm{C}_{70}$ fullerenes in various solvents", Fullerene Science and Technology 5:1593-1606, (1997).

[59] Ball, P. "Buckyball superconductors hot up", Nature, (2000).

[60] Cromie, W.J. "Carbon bits to revolutionize computer construction", Harvard University Gazette, 21 September, p. 1-4.

[61] Rotman, D. "Molecular Computing", Technology Review, May-June.

[62] "Space-age Soot", The Economist, 17 December.

[63] Dagani, R. "Building with BuckyBalls", Chemical and Engineering News 7742:54-59, (1999).

[64] Yeretzian, C., Wiley, J.B., Holczer, K., Su, T., Nguyen, S., Kaner, R.B., and Whettern, R.L. "Partial Separation of Fullerenes by Gradient Sublimation", Journal of Physical Chemistry 97:10097-10101, (1993).

[65] Liu, Y., O'Brien, S.C., Zhang, Q.L., Heath, J.R., Tittel, F.K., Curl, R.F., Kroto, H.W., and Smalley, R.E. "Negative carbon cluster ion beams: new evidence for the special nature of $\mathrm{C}_{60}$ ", Chemical Physics Letters 126:215-217, (1986).

[66] Curl, R.F. and Smalley, R.E. "Probing C60", Science 242:1017-1022, (1988).

[67] Zhang, Q.L., O'Brien, S.C., Heath, J.R., Liu, Y., Curl, R.F., Kroto, H.W., and Smalley, R.E. "Reactivity of Large Carbon Clusters: Spheroidal Carbon Shells and Their Possible Relevance to the Formation and Morphology of Soot", Journal of Physical Chemistry 90:525-528, (1986).

[68] Taylor, R. and Walton, D.R.M. "The chemistry of fullerenes", Nature 363:685693, (1993).

[69] Suzuki, T., Li, Q., Khemani, K.C., Wudl, F., and Almarsson, O. "Systematic Inflation of Buckminsterfullerene $\mathrm{C}_{60}$ : Synthesis of Diphenyl Fulleroids $\mathrm{C}_{61}$ to $\mathrm{C}_{66}$ ", Science 254:1186-1188, (1991).

[70] Rotello, V.M., Howard, J.B., Yadav, T., Conn, M.M., Viani, E., Giovane, L.M., and Lafleur, A.L. "Isolation of Fullerene Products from Flames: Structure and Synthesis of $\mathrm{C}_{60}$-Cyclopentadiene Adduct", Tetrahedron Letters 34:1561-1562, (1993).

[71] Giovane, L.M., Barco, J.W., Yadav, T., Lafleur, A.L., Marr, J.A., Howard, J.B., and Rotello, V.M. "Kinetic Stability of the $\mathrm{C}_{60}$-Cyclopentadiene Diels-Alder Adduct", Journal of Physical Chemistry 97:8560-8561, (1993).

[72] Krusic, P.J., Wasserman, E., Keizer, P.N., Morton, J.R., and Preston, K.F. "Radical Reactions of C60", Science 254:1183-1185, (1991). 
[73] Cataldo, F. "The role of fullerene-like structures in carbon black and their interaction with dienic rubber", Fullerene Science and Technology 8:105-112, (2000).

[74] Cataldo, F. "The role of fullerene-like structures in carbon black: theoretical consideration and experimental evidences", Third International Conference on Carbon Black, (2000).

[75] Yakobson, B.I. and Smalley, R.E. "Fullerene Nanotubes: $\mathrm{C}_{1,000,000}$ and Beyond", American Scientist 85:324-337, (1997).

[76] Dresselhaus, M.S., Dresselhaus, G., and Eklund, P.C., Science of Fullerenes and Carbon Nanotubes, Academic Press San Diego

[77] Ackerman, T. "Buckyball set to make mark in market/Uses are in treatment of AIDS, cancer", Houston Chronicle, 26 November, p. A37.

[78] Chen, B.-X., Wilson, S.R., Das, M., Couglin, D.J., and Erlanger, B.F. "Antigenicity of fullerenes: Antibodies specific for fullerenes and their characteristics", Proceedings of the National Academy of Sciences 10809-10813, (1998).

[79] Kouwenhoven, L. "Bouncing a $\mathrm{C}_{60}$ ball", Nature 407:35-36, (2000).

[80] Park, H., Park, J., Lim, A.K.L., Anderson, E.H., Alivisatos, A.P., and McEuen, P.L. "Nanochemical oscillations in a single $\mathrm{C}_{60}$-transistor", Nature 407:57-60, (2000).

[81] Gruen, D.M. "Nanocrystallline diamond films", Annual Review of Material Science 29:211-259, (1999).

[82] Lukich, L.T., Duncan, T.E., and Lansinger, C.M. "Use of fullerene carbon in curable rubber compounds", United States Patent \#5, 750,615; (1998).

[83] Long, R.Q. and Yang, R.T. "Carbon Nanotubes as Superior Sorbent for Dioxin Removal", Journal of the American Chemical Society 123:2058-2059, (2001).

[84] Gerhardt, P., Loffler, S., and Homann, K.-H. "Polyhedral Carbon Ions in Hydrocarbon Flames", Chemical Physics Letters 137:306-310, (1987).

[85] Howard, J.B., Mckinnon, J.T., Johnson, M.E., Makarovsky, Y., and Lafleur, A.L. "Production of $\mathrm{C}_{60}$ and $\mathrm{C}_{70}$ Fullerenes in Benzene-Oxygen Flames", Journal of Physical Chemistry 96:6657-6662, (1992).

[86] Howard, J.B., Lafleur, A.L., Makarovsky, Y., Mitra, S., Pope, C.J., and Yadav, T. "Fullerenes Synthesis in Combustion", Carbon 30:1183-1201, (1992).

[87] Howard, J.B. "Fullerenes Formation in Flames", Proceedings of the Combustion Institute 24:933-946, (1992).

[88] Richter, H., Taghizadeh, K., Grieco, W.J., Lafleur, A.L., and Howard, J.B. "Preparative-Scale Liquid Chromatography and Characterization of Large 
Fullerenes in Low-Pressure Benzene Flames", Journal of Physical Chemistry 100:19603-19610, (1996).

[89] Grieco, W.J., Lafleur, A.L., Swallow, K.C., Richter, H., Taghizadeh, K., and Howard, J.B. "Fullerenes and PAH in Low-Pressure Premixed Benzene/Oxygen Flames", Proceedings of the Combustion Institute 27:1669-1675, (1998).

[90] Hebgen, P. and Howard, J.B. "Fullerenes in Low Pressure Benzene/Oxygen Diffusion Flames", Joint Meeting of the British, German, and French Sections of the Combustion Institute, (1999).

[91] Hebgen, P. and Howard, J.B. "Synthesis of Fullerenes in Low Pressure Benzene/Oxygen Diffusion Flames", Fifth International Microgravity Combustion Workshop, (1999).

[92] Pope, C.J., Marr, J.A., and Howard, J.B. "Chemistry of Fullerenes $\mathrm{C}_{60}$ and $\mathrm{C}_{70}$ in Flames", Journal of Physical Chemistry 97:11001-11013, (1993).

[93] Baum, T., Loffler, S., Weilmunster, P., and Homann, K.-H. "Fullerene Ions and Their Relation to PAH and Soot in Low-Pressure Hydrocarbon Flames", Ber. Bunsenges. Phys. Chem. 96:841-857, (1992).

[94] Kronholm, D.F. and Howard, J.B. "Analysis of soot surface growth pathways using published plug-flow reactor data with new particle size distribution measurements and published premixed flame data", Proceedings of the Combustion Institute 28:2555-2561, (2000).

[95] Richter, H., Fonseca, A., Emberson, S.C., Gilles, J.-M., Nagy, J.B., Thiry, P.A., Caudano, R., and Lucas, A.A. "Fabrication of Fullerenes in Benzene/Oxygen/Argon and Benzene/Acetylene/Oxygen/Argon Flames", Journal Chimie Physica 92:1272-1285, (1995).

[96] Baum, T., Loffler, S., Weilmunster, P., and Homann, K.-H. The American Chemical Society, Division of Fuel Chemistry, 202 ${ }^{\text {nd }}$ ACS National Meeting 36:1533, (1991).

[97] Burden, A.P. and Hutchinson, J.L. "In situ fullerene formation - the evidence presented", Carbon 36:1167-1173, (1998).

[98] Gerhardt, P., Loffler, S., and Homann, K.-H. "The formation of polyhedral carbon ions in fuel-rich acetylene and benzene flames", Proceedings of the Combustion Institute 22:395-401, (1988).

[99] Grieco, W.J., Howard, J.B., Rainey, L.C., and Vander Sande, J.B. "Fullerenic Carbon in Combustion-Generated Soot", Carbon 38:597-614, (2000).

[100] Donnet, J.-B. "Black and White Fillers and Tire Compound", Rubber Chemistry and Technology 71:323-341, (1998).

[101] Donnet, J.-B., Wang, T.K., Wang, C.C., Monthious, M., Johnson, M.P., Norman, D.T., Wansborough, R.W., and Bertrand, P. "Carbon Black and Fullerenes - Part 
II: Precursor and Structure Identification", KGK Kautschuk Gummi Kunststoffe 5:340, (1999).

[102] Colbert, D.T., Zhang, J., McClure, S.M., Nikolaev, P., Chen, Z., Hafner, J.H., Owens, D.W., Kotula, P.G., Carter, C.B., Weaver, J.H., Rinzler, A.G., and Smalley, R.E. "Growth and Sintering of Fullerene Nanotubes", Science 266:1218, (1994).

[103] Diener, M., D., Nichelson, N., and Alford, J.M. "Synthesis of Single-Walled Carbon Nanotubes in Flames", Journal of Physical Chemistry 104, (2000).

[104] Richter, H., Hernadi, K., Caudano, R., Fonseca, A., Migeon, H.-N., Nagy, J.B., Schneider, S., Vandooren, J., and Van Tiggelen, P.J. "Formation of Nanotubes in Low Pressure Hydrocarbon Flames", Carbon 34:427-429, (1996).

[105] Bogdanov, A.A., Deininger, D., and Dyuzhev, G.A. "Development Prospects of the Commerical Production of Fullerenes", Technical Physics 45:521-527, (2000).

[106] Wong, R., President, SES Research, Houston, Texas, personal communication, 1999.

[107] Yadav, T. "Fullerene Synthesis and Processing, Part 1: Marketing Analysis", Proceedings of the Symposium on Recent Advances in Chemistry and Physics of Fullerenes and Related Materials, (1994).

[108] Withers, J.C., Loutfy, R.O., and Lowe, T.P. "Fullerene Commercial Vision", Fullerene Science and Technology 5, (1997).

[109] Yadav, T. "Fullerene Synthesis and Processing, Part 2: Engineering Analysis", Proceedings of the Symposium on Recent Advances in Chemistry and Physics of Fullerenes and Related Materials, (1994).

[110] Richter, H., Emberson, S.C., and Fonseca, A. "Fabrication Methods for Fullerenes: A Critical Review", Revue de Institut Francaise du Petrole, (1994).

[111] Wright, J.D., Haufler, R.E., Alford, J.M., Bell, W.L., and Copeland, R.J. "Combustion Synthesis of Fullerenes: Engineering Analysis and Cost of Production", Electrochemical Society Meeting, (1994).

[112] Richter, H., de Hoffman, E., Doome, R.J., Fonseca, A., Gilles, J.-M., Nagy, J.B., Thiry, P.A., Vandooren, J., and Van Tiggelen, P.J. "Fullerene Formation in Acetylene/Oxygen/Argon/Chlorine Flames", Carbon 34:797-803, (1996).

[113] Anacleto, J.F., Quilliam, M.A., Boyd, R.K., Howard, J.B., Lafleur, A.L., and Yadav, T. "Charge-transfer Ionspray Liquid Chromatography/Mass Spectrometry Analyses of Fullerenes and Related Compounds from Flame-Generated Materials", Rapid Communications in Mass Spectrometry 7:229-234, (1993).

[114] Anacleto, J.F., Boyd, R.K., Pleasance, S., Quilliam, M.A., Howard, J.B., Lafleur, A.L., and Makarovsky, Y. "Analysis of minor constituents in fullerene soots by 
LC-MS using a heated pneumatice nebulizer interface with atmospheric pressure chemical ionization", Canadian Journal of Chemistry 70:2558-2568, (1992).

[115] Bittner, J.D. and Howard, J.B. "Composition profiles and reaction mechanisms in a near-sooting premixed benzene/oxygen/argon flame", Proceedings of the Combustion Institute 18:1105-1116, (1981).

[116] Lafleur, A.L., Howard, J.B., Marr, J.A., and Yadav, T. "Proposed Fullerene Precursor Corannulene Identified in Flames Both in the Presence and Absence of Fullerene Production", Journal of Physical Chemistry 97:13539-13543, (1993).

[117] Mckinnon, J.T. "Chemical and Physical Mechanisms of Soot Formation", Ph.D. Thesis, Massachusetts Institute of Technology (1989).

[118] Hebgen, P., Post-doctoral associate, Massachusetts Institute of Technology, Cambridge, Massachusetts, personal communication, 2000.

[119] Mochida, I., Egashira, M., Koura, H., Dakeshita, K., Yoon, S.-H., and Korai, Y. "Carbonization of $\mathrm{C}_{60}$ and $\mathrm{C}_{70}$ Fullerenes to Fullerene Soot", Carbon 33:1186$1188,(1995)$.

[120] Mochida, I., Egashira, M., Korai, Y., and Yokogawa, K. "Structural changes of fullerene by heat-treatment up to graphitization temperature", Carbon 35:17071712, (1997).

[121] Fuller, T. and Banhart, F. "In situ observation of the formation and stability of single fullerene molecules under electron irradiation", Chemical Physics Letters 254:372-378, (1996).

[122] Ismail, I.M.K. and Rogers, S.L. "Comparisons between fullerene and forms of well-known carbons", Carbon 30:229-239, (1992).

[123] Kolodney, E., Tsipinyuk, B., and Budrevich, A. "The thermal stability and fragmentation of $\mathrm{C}_{60}$ molecule up to $200 \mathrm{~K}$ on the milliseconds time scale", Journal of Chemical Physics 100:8542-8545, (1994).

[124] Sommer, T., Kruse, T., and Roth, P. " $\mathrm{C}_{2}$ Formation during High-Temperature Pyrolysis of Fullerene $\mathrm{C}_{60}$ in Shock Waves", Journal of Physical Chemistry 99:13509-13512, (1995).

[125] Whetten, R.L. and Yeretzian, C. "Fullerenes Under Extreme Temperatures and Stress: Collisions of Fullerenes with Surfaces and With Other Fullerenes", International Journal of Modern Physics B 6:3801-3814, (1992).

[126] Cox, D.M., Behal, S., Disko, M., Gorun, S.M., Gleaney, M., Hsu, C.S., Kollin, E.B., Millar, J., Robbins, J., Robbins, W., Sherwood, R.D., and Tindall, P. "Characterization of $\mathrm{C}_{60}$ and $\mathrm{C}_{70}$ clusters", Journal of the American Chemical Society 113:2940-2944, (1991).

[127] Ajayan, P.M. and Iijima, S. "Smallest carbon nanotube", Nature 358:23, (1992). 
[128] Meier, M., Department of Chemistry, University of Kentucky, Lexington, Kentucky, personal communication, 2001.

[129] Wang, S. and Buseck, P.R. "Radiation damage of $\mathrm{C}_{60}$ crystals", Carbon 31:297298, (1993).

[130] Donnet, J.-B., Bansal, R.C., and Wang, M.-J., Carbon Black: Second Edition, Revised and Expanded, Marcell Dekker, Inc. New York, New York (1993).

[131] Energy Prices \& Taxes Quarterly Statistics (Third Quarter 2001), International Energy Agency (2001).

[132] Lalvani, S.B., Wiltowski, T., Hubner, A., Weston, A., and Mandich, N. "Removal of hexavalent chromium and metal cations by a selective and novel carbon adsorbent", Carbon 36:1219-1226, (1998).

[133] Mandich, N.V., Lalvani, S.B., Wiltowski, T., and Lalvani, L.S. "Selective Removal of Chromate Anion by a New Carbon Adsorbent", Metal Finishing May:39-44, (1998). 


\section{Appendices}

\subsection{Appendix 1-Tabulation of high-flow (turbulent) flame data}

Flame \#11

\begin{tabular}{|c|c|c|c|}
\hline $\begin{array}{c}\text { Height above } \\
\text { burner }(\mathrm{mm})\end{array}$ & $\begin{array}{c}\text { Percent of } \\
\text { condensable material }\end{array}$ & $\begin{array}{c}\text { Concentration } \\
\text { (ug/L) }\end{array}$ & C60/C70 \\
\hline \hline 352 & 2.82 & 1877.37 & 1.07 \\
\hline 367 & 1.05 & 266.91 & 0.92 \\
\hline 382 & 1.39 & 356.34 & 1.81 \\
\hline 406 & 0.93 & 175.39 & 1.55 \\
\hline 418 & 0.79 & 155.33 & 1.67 \\
\hline 436 & 0.52 & 104.16 & 1.30 \\
\hline 457 & 0.10 & 13.52 & 1.13 \\
\hline 482 & 0.45 & 36.61 & 0.95 \\
\hline 507 & 0.22 & 29.93 & 0.90 \\
\hline 531 & 2.02 & 99.45 & 0.94 \\
\hline
\end{tabular}

Flame \#12

\begin{tabular}{|c|c|c|c|}
\hline $\begin{array}{c}\text { Height above } \\
\text { burner }(\mathrm{mm})\end{array}$ & $\begin{array}{c}\text { Percent of } \\
\text { condensable material }\end{array}$ & $\begin{array}{c}\text { Concentration } \\
(\mathrm{ug} / \mathrm{L})\end{array}$ & $\mathrm{C60/C70}$ \\
\hline \hline 382 & 0.18 & 32.17 & 0.93 \\
\hline 400 & 1.70 & 200.38 & 1.16 \\
\hline 419 & 0.73 & 135.73 & 1.07 \\
\hline 441 & 1.60 & 174.88 & 1.00 \\
\hline 462 & 1.83 & 245.06 & 0.89 \\
\hline 482 & 0.76 & 143.41 & 0.88 \\
\hline 507 & 1.54 & 195.07 & 0.80 \\
\hline
\end{tabular}

Flame \#13

\begin{tabular}{|c|c|c|c|}
\hline $\begin{array}{c}\text { Height above } \\
\text { burner }(\mathrm{mm})\end{array}$ & $\begin{array}{c}\text { Percent of } \\
\text { condensable material }\end{array}$ & $\begin{array}{c}\text { Concentration } \\
(\mathrm{ug} / \mathrm{L})\end{array}$ & $\mathrm{C60/ \textrm {C70 }}$ \\
\hline \hline 307 & 0.62 & 256.79 & 1.15 \\
\hline 324 & 0.33 & 110.31 & 2.16 \\
\hline 341 & 0.36 & 104.28 & 0.77 \\
\hline 358 & 0.42 & 116.97 & 0.61 \\
\hline 375 & 0.63 & 127.90 & 0.98 \\
\hline 392 & 1.18 & 255.83 & 0.62 \\
\hline
\end{tabular}

Flame \#14

\begin{tabular}{|c|c|c|c|}
\hline $\begin{array}{c}\text { Height above } \\
\text { burner }(\mathrm{mm})\end{array}$ & $\begin{array}{c}\text { Percent of } \\
\text { condensable material }\end{array}$ & $\begin{array}{c}\text { Concentration } \\
(\mathrm{ug} / \mathrm{L})\end{array}$ & $\mathrm{C60/ \textrm {C } 7 0}$ \\
\hline \hline 362 & 1.00 & 306.97 & 1.87 \\
\hline 382 & 1.44 & 376.23 & 0.75 \\
\hline 402 & 1.63 & 453.08 & 1.55 \\
\hline 422 & 1.08 & 242.98 & 0.60 \\
\hline 442 & 0.85 & 140.15 & 2.72 \\
\hline 462 & 2.99 & 375.13 & 1.06 \\
\hline
\end{tabular}




\subsection{Appendix 2-Premixed flame data and sample calculation}

McKinnon Flame
\begin{tabular}{|c|c|c|c|}
$\begin{array}{c}\text { Height above } \\
\text { nozzle } \\
(\mathrm{mm})\end{array}$ & $\begin{array}{c}\text { Flame Concentration } \\
\left(\mathrm{ng} / \mathrm{cm}^{3}\right)\end{array}$ & $\begin{array}{c}\text { Soot Conc. } \\
\text { in flame } \\
\left(\mathrm{ng} / \mathrm{cm}^{3}\right)\end{array}$ \\
\hline 5.8 & 0 & 0 & 102.73 \\
\hline 12 & 0.47 & 0.63 & 113.93 \\
\hline 20 & 1.24 & 1.36 & 118.89 \\
\hline 30 & 3.06 & 3.08 & 126.95 \\
\hline 40 & 3.50 & 3.42 & 149.20 \\
\hline 50 & 3.62 & 3.35 & 117.39 \\
\hline 60 & 4.85 & 4.59 & 170.80 \\
\hline 70 & 5.48 & 5.22 & 173.69 \\
\hline 80 & 4.58 & 4.16 & 171.39 \\
\hline 90 & 5.05 & 3.82 & 224.98 \\
\hline 100 & 6.94 & 6.38 & 269.22 \\
\hline 110 & 7.32 & 6.59 & 294.82 \\
\hline 120 & 9.37 & 8.11 & 309.11 \\
\hline
\end{tabular}

Two-stage flames - all data are flame concentration $\left(\mathrm{ng} / \mathrm{cm}^{3}\right)$

\begin{tabular}{|c|c|c|c|c|c|c|c|c|c|}
\hline Height above & \multicolumn{3}{|c|}{ Flame \#1 } & \multicolumn{3}{c|}{ Flame \#2 } & \multicolumn{3}{c|}{ Flame \#3 } \\
burner (mm) & $\mathrm{C}_{60}$ & $\mathrm{C}_{70}$ & Soot & $\mathrm{C}_{60}$ & $\mathrm{C}_{70}$ & Soot & $\mathrm{C}_{60}$ & $\mathrm{C}_{70}$ & Soot \\
\hline \hline 15 & & & & & & & 0.00 & 0.00 & 680.18 \\
\hline 20 & & & & & & & 0.00 & 0.00 & 254.77 \\
\hline 30 & & & & & & & 0.00 & 0.00 & 183.11 \\
\hline 35 & 2.38 & 2.34 & 160.70 & 0.00 & 0.00 & 188.09 & & & \\
\hline 40 & 2.26 & 2.31 & 179.53 & 0.00 & 0.00 & 192.96 & 0.00 & 0.00 & 297.83 \\
\hline 50 & 0.98 & 0.76 & 237.71 & 0.00 & 0.00 & 156.07 & 0.00 & 0.00 & 307.14 \\
\hline 60 & 0.43 & 0.38 & 202.81 & 0.00 & 0.00 & 155.69 & 0.00 & 0.00 & 274.71 \\
\hline 70 & 0.58 & 0.49 & 153.38 & 0.00 & 0.00 & 135.39 & & & \\
\hline 80 & 0.87 & 0.71 & 151.89 & 0.00 & 0.00 & 115.96 & & & \\
\hline 90 & 1.72 & 0.93 & 146.82 & 0.00 & 0.00 & 88.95 & & & \\
\hline 100 & 1.94 & 1.93 & 175.53 & 0.00 & 0.00 & 81.93 & & & \\
\hline 110 & 2.31 & 2.08 & 197.81 & 0.00 & 0.00 & 82.57 & & & \\
\hline 120 & 3.01 & 3.08 & 230.86 & 0.00 & 0.00 & 79.06 & & & \\
\hline
\end{tabular}

\begin{tabular}{|c|c|c|c|c|c|c|c|c|c|}
\hline Height above & \multicolumn{3}{|c|}{ Flame \#4 } & \multicolumn{3}{|c|}{ Flame \#5 } & \multicolumn{3}{|c|}{ Flame \#6 } \\
burner (mm) & $\mathrm{C}_{60}$ & $\mathrm{C}_{70}$ & Soot & $\mathrm{C}_{60}$ & $\mathrm{C}_{70}$ & Soot & $\mathrm{C}_{60}$ & $\mathrm{C}_{70}$ & Soot \\
\hline \hline 30 & & & & 0.00 & 0.00 & 3.727 & & & \\
\hline 35 & 0.91 & 0.72 & 100.64 & 0.00 & 0.00 & -0.172 & 0.00 & 0.00 & 3.83 \\
\hline 35 & 0.95 & 0.72 & 76.97 & & & & & & \\
\hline 40 & 1.25 & 1.17 & 105.63 & 0.00 & 0.00 & 0.307 & 0.03 & 0.02 & 6.46 \\
\hline 40 & 0.95 & 0.92 & 64.55 & & & & & & \\
\hline 50 & 0.28 & 0.32 & 50.23 & 0.00 & 0.00 & 0.924 & 0.15 & 0.13 & 3.89 \\
\hline 60 & 0.77 & 0.60 & 43.41 & 0.01 & 0.01 & -0.609 & 0.17 & 0.13 & 1.00 \\
\hline 60 & 0.85 & 0.64 & 74.18 & & & & & & \\
\hline 70 & 1.91 & 1.48 & 43.61 & 0.14 & 0.08 & 1.149 & 0.20 & 0.12 & 1.16 \\
\hline 70 & 2.62 & 1.62 & 77.72 & & & & & & \\
\hline 70 & 2.10 & 1.32 & 88.93 & & & & & & \\
\hline 80 & 2.69 & 1.45 & 77.40 & & & & & & \\
\hline
\end{tabular}


Sample calculation for data at $70 \mathrm{~mm}$ height above nozzle in the Mckinnon flame:

Soot collected: $\quad 11.62 \mathrm{mg} \quad$ (measured by weight)

$\mathrm{C}_{60}$ collected: $\quad 366.92 \mu \mathrm{g} \quad$ (measured by HPLC)

$\mathrm{C}_{70}$ collected: $\quad 349.11 \mu \mathrm{g} \quad$ (measured by HPLC)

Sample gas collected: $\quad 758 \mathrm{~cm}^{3} \quad$ (measured in water column)

Lab temperature: $\quad 297.65 \mathrm{~K}$

Water column pressure: $\quad \mathbf{7 2 9 . 4}$ Torr

Flame pressure: $\quad 40$ Torr $\quad$ (experiment control)

Flame temperature at $70 \mathrm{~mm}: 1440 \mathrm{~K} \quad$ (from reference [37])

Adjust gas volume to flame conditions:

Volume of gas in chamber $=758 \mathrm{~cm}^{3} \times \frac{729.4 \text { Torr }}{40 \text { Torr }} \times \frac{1440 \mathrm{~K}}{297.65 \mathrm{~K}}=66900 \mathrm{~cm}^{3}$

Divide each mass value by gas volume to get the concentration:

$\mathrm{C}_{60}: \quad C_{60}$ concentration $=366.92 \mu \mathrm{g} \times \frac{1000 \mathrm{ng}}{1 \mu \mathrm{g}} \times \frac{1}{66900 \mathrm{~cm}^{3}}=5.48 \frac{\mathrm{ng}}{\mathrm{cm}^{3}}$

$\mathrm{C}_{70}: \quad C_{70}$ concentration $=349.11 \mu \mathrm{g} \times \frac{1000 \mathrm{ng}}{1 \mu \mathrm{g}} \times \frac{1}{66900 \mathrm{~cm}^{3}}=5.22 \frac{\mathrm{ng}}{\mathrm{cm}^{3}}$

Soot: $\quad$ Soot concentration $=11.62 \mathrm{mg} \times \frac{1000000 \mathrm{ng}}{1 \mathrm{mg}} \times \frac{1}{66900 \mathrm{~cm}^{3}}=173.69 \frac{\mathrm{ng}}{\mathrm{cm}^{3}}$ 


\subsection{Appendix 3-Calculations for fullerenic soot yield}

\subsubsection{Calculations from observed experimental data}

Data used below are taken from a height above burner of $120 \mathrm{~mm}$ in the regular McKinnon flame data (Figure 4-6). This data point was used because it is at the longest residence time and hence, closest to equilibrium. At this point, the soot concentration is $309.11 \mathrm{ng} / \mathrm{cm}^{3}$. The subsequent calculations are as follows:

Soot Conc. $=309.11 \frac{\mathrm{ng}}{\mathrm{cm}^{3}}$ at flame conditions

Soot Conc $=309.11 \frac{\mathrm{ng}}{\mathrm{cm}^{3}} \times \frac{760 \text { Torr }}{40 \text { Torr }} \times \frac{869.5 \mathrm{~K}}{273.15 \mathrm{~K}}=18695 \frac{\mathrm{ng}}{\mathrm{cm}^{3}} \quad$ at standard conditions

Gas Flow Rate $=2.785 S L P=2785 s c c m$ assuming no mole change

Soot Prod Rate $=18695 \frac{\mathrm{ng}}{\mathrm{cm}^{3}} \times 2785 \frac{\mathrm{cm}^{3}}{\mathrm{~min}}=52066722 \frac{\mathrm{ng}}{\mathrm{min}}=54.067 \frac{\mathrm{mg}}{\mathrm{min}}$

Carbon Feed Rate $=0.608 \frac{\mathrm{L}}{\mathrm{min}} \times \frac{1 \mathrm{~atm}}{0.08206 \frac{\mathrm{Latm}}{\mathrm{molK}} \times 273.15 \mathrm{~K}} \times 72 \frac{\mathrm{g}}{\mathrm{mol}}=1.952 \frac{\mathrm{g}}{\mathrm{min}}$

from benzene

Soot Yield $=\frac{52.067 \frac{\mathrm{mg}}{\mathrm{min}}}{1952 \frac{\mathrm{mg}}{\mathrm{min}}}=0.02667 \frac{\mathrm{mg} \text { soot }}{\mathrm{mg} \text { carbon fed }}=2.67 \% \approx 3 \%$

So the assumed observed fullerenic soot yield is approximately $3 \%$. 


\subsubsection{Thermodynamic equilibrium calculations by STANJAN}

A STANJAN equilibrium calculation was run at constant temperature and pressure with the following inputs in the following table. The inputs correspond to those present in the McKinnon flame. The Chemical Kinetics Library (CKLIB) in conjunction with CHEMKIN-II Version 4.5 was used for predicting equilibrium concentrations in this calculation.

Input Parameters

\begin{tabular}{|l|l|}
\hline Parameter & Value \\
\hline Pressure & $0.0526 \mathrm{~atm}$ \\
\hline Temperature & $1440 \mathrm{~K}$ \\
\hline Initial benzene & $0.2182 \mathrm{~mol}$ fraction \\
\hline Initial oxygen & $0.6818 \mathrm{~mol}$ fraction \\
\hline Initial argon & $0.1000 \mathrm{~mol}$ fraction \\
\hline
\end{tabular}

The important output parameters from the calculation are summarized in the next table. STANJAN calculates equilibrium values for the physical properties of the system as well as the mole fractions of all species involved. The table shows the ones that are used to calculate the soot yield.

Output Parameters

\begin{tabular}{|l|l|}
\hline Parameter & Value \\
\hline Initial average molecular weight & $4.286 \mathrm{~g} / \mathrm{mol}$ \\
\hline Final average molecular weight & $2.077 \mathrm{~g} / \mathrm{mol}$ \\
\hline Initial benzene & $0.2182 \mathrm{~mole}$ fraction \\
\hline Final solid carbon & 0.0195 mole fraction \\
\hline
\end{tabular}


The soot yield can be calculated from these values by calculating the moles of carbon fed and moles of solid carbon produced and comparing the two. The calculations follow:

Carbon Fed $=0.2182 \times 6 \frac{\mathrm{mol} \text { Carbon }}{\text { mol Benzene }}=1.3092 \mathrm{~mol}$

$$
\text { Solid Carbon Made }=0.019456 \times \frac{42.856 \frac{\mathrm{g}}{\mathrm{mol}}}{20.766 \frac{\mathrm{g}}{\mathrm{mol}}}=0.04015 \mathrm{~mol}
$$

Soot Yield $=\frac{0.04015 \mathrm{~mol}}{1.3092 \mathrm{~mol}}=0.030669=3.0669 \% \approx 3 \%$

So this confirms the observed fullerenic soot yield of approximately $3 \%$. 


\subsection{Appendix 4-Economic cost model for fullerenic soot plant}

\subsubsection{Spreadsheet for economic cost model - base case cost}

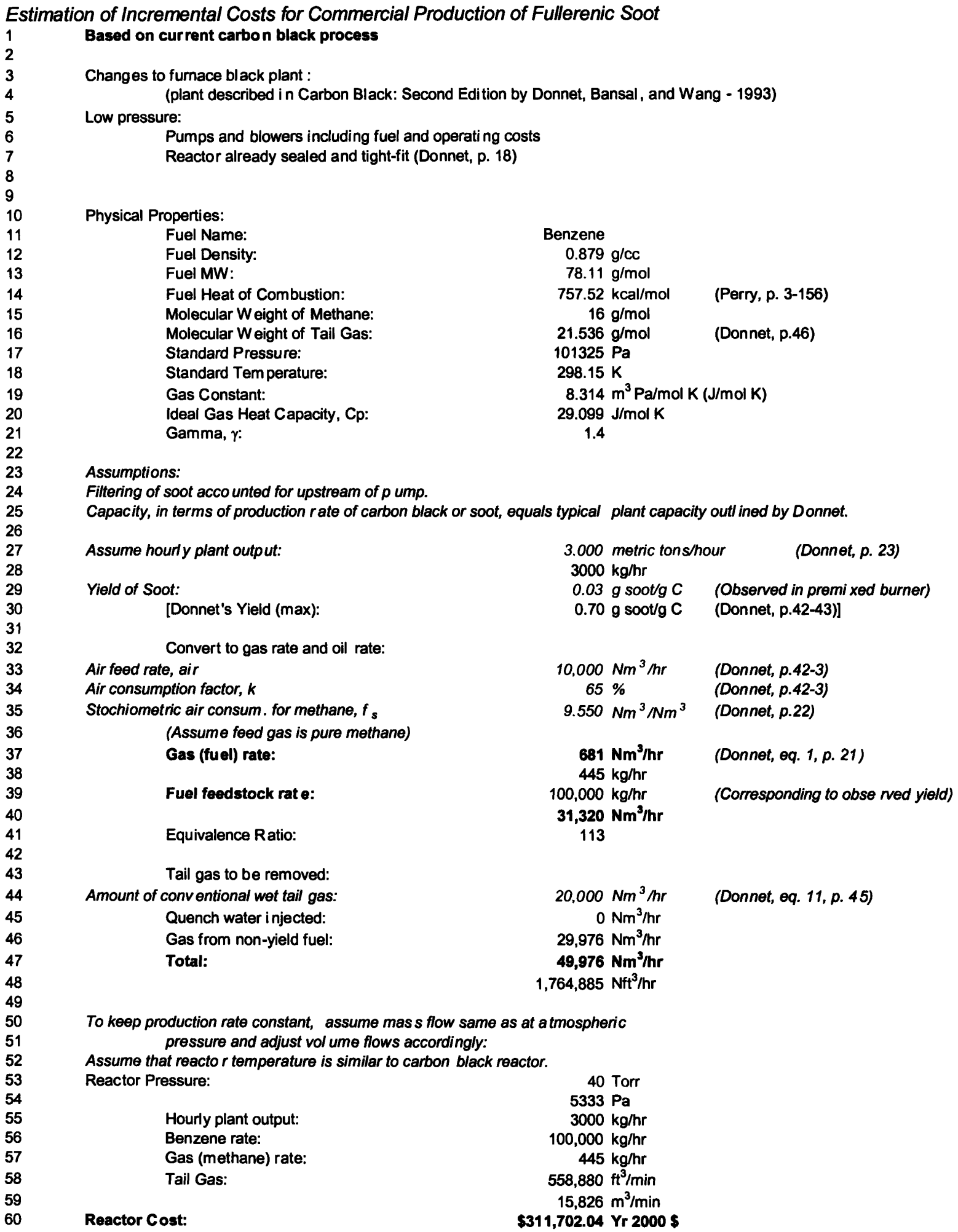


Pump Price Estimate for 12000 scfm capacity:

Dekker Vacuum Technology:

175,000 Yr2000\$

(Dekker Technology)

From Peters and Timmerhaus (1980):

Ten (10) Rotary compressors in parallel each with a capacity of 1200 scfm:

110,000 Yr1979\$

196,051 Yr1999\$

201,933 Yr2000s

Nash Engineering Company:

170,000 Yr2000s

Average

Pumps Needed:

$\$ 182,311$ Yr2000s

(Peters et al., p. 559)

(Marshall \& Swift)

(3\% inflation)

(Nash Engineering)

Power Requirements:

Assume adiabatic compression.

Assume tail gas is ideal.

Supplied Power:

Per hour

Pump Efficiency:

Power Required:

Heat of comb of conventional tail gas:

Heat of comb of low product yield tail gas:

Tail Gas Conversion Efficiency

Power converted from tail gas:

Power to be bought:

Excess power from tail gas:

Cost of Electricity:

Fuel Cost:

Generation Cost:

Power Cost:

\author{
$24,625 \mathrm{~J} / \mathrm{m}^{3}$ \\ $23,382,667 \mathrm{~kJ} / \mathrm{hr}$ \\ $80 \%$ \\ $29,228,334 \mathrm{~kJ} / \mathrm{hr}$ \\ $2500 \mathrm{~kJ} / \mathrm{m}^{3}$ \\ $3141 \mathrm{~kJ} / \mathrm{mol}$ \\ $40 \%$ \\ $1,559,631,685 \mathrm{~kJ} / \mathrm{hr}$ \\ $0 \mathrm{~kJ} / \mathrm{hr}$ \\ $1,530,403,351 \mathrm{~kJ} / \mathrm{hr}$
}

(McCabe, p. 209)

0.0267 Yr2000\$/kWhr

0.0146 Yr2000s/kWhr

$\$ 118.32$ Yr2000\$/hr

Translate Costs to per kg basis:

Pump lifetime:

Hours operation per day:

Day operation per year:

Total Cost:

Total Production:

Cost per kg:

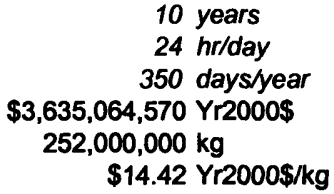

Total Incremental Cost

for fullerenic soot:

\title{
$\$ 14.42486$ Yr2000s/kg
}

$\$ 6.54301$ Yr2000\$/lb

References:

Dekker Vacuum Technologies, Inc., Personal Communication, 20 April 2000.

Department of Energy Weekly Petroleum Status Report

(as reported in Oil \& Gas Journal, 10 Jully 2000, p. 89).

Donnet, J.-B., Bansal, R.C., Wang, M.-J. Carbon Black: Second Edition, Revised and Expanded, Marcel Dekker, Inc., New York 1993.

Energy Information Administration, Form ElA-826, "Monthly Electric Utility Sales and Revenue Report with State Distributions."

Marshall and Swift Equipment Cost Indices (taken from the journal Chemical Engineering for 1979-1999).

McCabe, W.L., Smith, J.C., Harriot, P. Unit Operations of Chemical Engineering: Fifth Edition McGraw-Hill, Inc., New York, 1993.

Nash Engineering Company, Facsimilie Communication, 16 May 2000.

Perry, R.H., Green, D.W., Maloney, J.O. Perry's Chemical Engineers' Handbook: Sixth Edition, McGraw-Hill, Inc., New York, 1984.

Peters, M.S., Timmerhaus, K.D. Plant Design and Economic for Chemical Engineers: Third Edition, McGraw-Hill, Inc., New York, 1980. 


\subsubsection{Explanation of cost model}

This section explains the entries in the economic cost model spreadsheet presented in Section 11.2.1. This appendix is meant to provide only explanations behind the numbers as the assumptions have all been discussed in Section 7.2. Some things to note globally about the spreadsheet are:

- Assumptions are in italics

- Major section headings and final costs begin in the first column of text

- Parameters, constants, and calculated values start in the second column

- Units are immediately to the right of the value they describe

- If used, the reference for the value follows to the right (the full list of references is given at the end of the model)

- Values in bold are important or final section parameters

- All dollars and cents have been converted into Year2000 figures.

The values that need further explanation or clarification are outlined below:

Lines 33-35: These are parameters necessary for the calculation of gas and feedstock federates. The air consumption factor is the percentage of total oxygen used for complete combustion.

Lines 37-40: Gas (fuel) is the primary flame fuel source used for energy generation and goes to complete combustion (here, we use methane). Fuel feedstock is the liquid secondary fuel used for product formation (benzene here).

Line 45: No quench water is injected as combustion will already be incomplete due to the high equivalence ratio (line 41).

Line 46: This gas is produced solely from the excess benzene feed and was treated as tail gas additional to that produced by the conventional gases (which is also present in line 44).

Line 60: The reactor cost was estimated by using a scaling factor given by Peters \& Timmerhaus in 1980. The ratio of reactor volumes was used to calculate the cost of the new reactor. The original reactor price was subtracted to get the incremental cost. 
Lines 62-63: The fuel cost for benzene was converted to an hourly cost by incorporating the feedstock flowrate and subtracting out the original flowrate.

Lines 65-73: Three pump price estimates were made; two were quotes obtained from outside commercial companies, and one was calculated from an engineering design text (Peters \& Timmerhaus). The average was taken as a fairly accurate pump cost.

Lines 74: The number of pumps needed was obtained by comparing the tail gas volume in Line 48 to the $12000 \mathrm{scfm}$ capacity.

Lines 80-81: The power requirement was obtained using gas pressures and heat capacities and then converted to an hourly basis using the volumetric flowrate (line 59).

Line 87: The potential power recovered from the tail gas was calculated using the heats of combustion in lines $84 \& 85$ with respective flowrates in lines 44 \& 46.

Lines 88-89: These lines compare the power required in line 83 to that available in the tail gas in line 87 and reports the amount to be bought or the excess depending on their relative magnitudes. In theory, any excess power could be sold but that is not accounted for here.

Lines 90-93: The power required is combined with the fuel and generation costs to get the final power cost. Only power to be bought is subject to the fuel cost but all of the power is subject to the generation cost.

Line 99: The total cost is found by adding the pump and reactor costs (capital costs) to the fuel and electricity costs (operating costs) on a project lifetime basis.

Line 100: Total production is also calculated for the duration of the project lifetime.

Line 105: Final incremental cost to producing fullerenic soot instead of carbon black on a per pound basis. 